Aus der

Klinik für Psychiatrie und Psychotherapie des Westfälischen Zentrum Bochum

- Universitätsklinik -

der Ruhr-Universität Bochum

ehem. Direktor: Prof. Dr. med. P. L. Janssen

\title{
Psychotherapie und Internet
}

INAUGURAL-DISSERTATION

zur

Erlangung des Doktorgrades der Medizin

einer

Hohen Medizinischen Fakultät

der Ruhr-Universität Bochum vorgelegt von

Christoph Barthel

aus Hamm

2008 
Dekan: Prof. Dr. med. Muhr

1. Referent: Prof. Dr. med P.L. Janssen

2. Referent: Prof. Dr. med S. Herpertz

Tag der mündlichen Prüfung: 22.01.2009 


\section{INHALTSVERZEICHNIS}

ABBILDUNGSVERZEICHNIS $\quad 3$

TABELLENVERZEICHNIS

EINLEITUNG 6

1. DAS INTERNET - GESCHICHTE UND VERBREITUNG 7

1.1 DIE GESCHICHTE DES INTERNETS

1.2 INTERNETNUTZUNG HEUTE

1.3 KOMMUNIKATIONSMÖGLICHKEITEN VIA INTERNET 15

2. COMPUTERVERMITTELTE KOMMUNIKATION 20

2.1 EIGENSCHAFTEN COMPUTERVERMITTELTER KOMMUNIKATION 20

2.1.1 SYNCHRONITÄT UND ASYNCHRONITÄT 20

2.1.2 ENTKÖRPERLICHUNG UND ANONYMITÄT 21

2.1.3 TEXTUALITÄT 21

2.1.4 ENTZEITLICHUNG UND ENTRÄUMLICHUNG 22

2.1.5 ENTKONTEXTUALISIERUNG 23

2.1.6 DigitALISIERUNG 24

2.2 THEORIEN COMPUTERVERMITTELTER KOMMUNIKATION 24

2.2.1 SOCIAL PRESENCE THEORY 25

2.2.2 MEDIA RICHNESS THEORY 26

2.2.3 LACK OF SOCIAL CONTEXT AND CUES THEORY 27

3. DIE ROLLE DES INTERNETS IN DER KLINISCHEN PSYCHOLOGIE UND PSYCHOTHERAPIE $\quad 30$

4. INTERNETBASIERTE KLINISCH-PSYCHOLOGISCHE INTERVENTION 33

4.1. ENTWICKLUNG UND STATUS QUO 33

4.2. QUALITÄTSANFORDERUNGEN AN ONLINE-ANGEBOTE Z. THERAPEUT. INTERVENTION 35

4.3 SELBSTHILFE 37

4.4 ONLINE-BERATUNG 38

4.4.1 ANGEBOT PSYCHOLOGISCHER ONLINE-BERATUNG 40

4.4.2 E-MAIL-BERATUNG DER KATHOLISCHEN TELEFONSEELSORGE 41

5. EIGENES PROJEKT

6. ONLINE-THERAPIE 46

6.1 THERAP-E-MAIL

6.2 INTERAPY 48

6.3 THERATALK $\quad 49$

6.4 NEUE ENTWICKLUNGEN: VON DER INTERNETBASIERTEN Z. MOBILEN INTERVENTION $\quad 50$

7. EMPIRISCHE BEFUNDE ZUR ONLINE-THERAPIE 
7.1 ÜBERBLICK ÜBER DIE BISHERIGE FORSCHUNG 53

7.2 AUSGEWÄHLTE STUDIEN 56

7.2.1 BEHANDLUNG VON POSTTRAUMATISCHEN BELASTUNGSSTÖRUNGEN MIT DEM INTERAPY-

$\begin{array}{ll}\text { VERFAHREN } & 57\end{array}$

7.2.2 PAARTHERAPIE NACH DEM THERATALK-KONZEPT

7.2.3 INTERNETCHATGRUPPEN ZUR NACHBEHANDLUNG 62

7.2.4 NACHBEHANDLUNG BEI BULIMIA NERVOSA MIT HILFE VON TEXTNACHRICHTEN 65

8. DISKUSSION: MÖGLICHKEITEN UND GRENZEN DER ONLINE-THERAPIE 67

9. ZUSAMMENFASSUNG

$\begin{array}{ll}\text { LITERATURVERZEICHNIS } & 74\end{array}$

$\begin{array}{lr}\text { ANHANG } & 83\end{array}$

A 1: TABELLE ZUR WELTWEITEN INTERNETNUTZUNG $\quad 84$

A 2 : BEISPIELE FÜR INTERNETSEITEN 85

A 3: LISTE ZERTIFIZIERTER ONLINE-BERATER 86

A 4: CHECKLISTE FÜR NUTZER VON SUCHTHILFEANGEBOTEN IM INTERNET

A 5: AUSWAHL DEUTSCHSPRACHIGER ONLINE-BERATUNGSANGEBOTE 89

A 6: LISTE DER IM LITERATUR-REVIEW BERÜCKSICHTIGTEN STUDIEN 90 


\section{Abbildungsverzeichnis}

\begin{tabular}{|c|c|c|}
\hline Abbildungsnummer & Abbildungsbeschreibung & Seite \\
\hline Abb. 1 & Netzwerkstrukturen (nach Baran, 1964, S. 2) & 9 \\
\hline Abb. 2 & $\begin{array}{l}\text { Internetnutzer weltweit in Millionen (nach Brym \& } \\
\text { Lenton, 2001, S. 6) }\end{array}$ & 12 \\
\hline Abb.3 & $\begin{array}{l}\text { Entwicklung der Internetnutzung in Deutschland von } \\
1997 \text { bis } 2006 \text { (nach den Ergebnissen von Eimeren \& } \\
\text { Frees, 2006) }\end{array}$ & 14 \\
\hline Abb. 4 & $\begin{array}{l}\text { Wichtigkeit von Kommunikationsformen im } \\
\text { Vergleich, Prozentzahlen geben an, welcher Anteil } \\
\text { der Befragten die jeweilige Kommunikationsform als } \\
\text { „wichtig“ oder sehr wichtig“ eingestuft haben (nach } \\
\text { Fittkau \& Maaß, 2004) }\end{array}$ & 15 \\
\hline Abb. 5 & $\begin{array}{l}\text { Hierarchie der Media Richness (nach Daft, Lengel \& } \\
\text { Trevino, 1987) }\end{array}$ & 26 \\
\hline Abb. 6 & $\begin{array}{l}\text { Schnittstellen der Klinischen Psychologie mit dem } \\
\text { Medium Internet; die Zahlenangaben der Felder } \\
\text { stehen für } 1 \text { = Informationsangebote; } 2 \text { = Forschung, } \\
3 \text { = Intervention und Selbsthilfe, } 4=\text { Aspekte und } \\
\text { Effekte der Internetnutzung (modifiziert nach Ott, R. } \\
\text { \& Eichenberg, C., 2003, S. 14) }\end{array}$ & 31 \\
\hline Abb. 7 & $\begin{array}{l}\text { Vorgehensweise bei der Literaturanalyse (Ott, 2003, } \\
\text { S. 130) }\end{array}$ & 54 \\
\hline Abb. 8 & $\begin{array}{l}\text { Anzahl der Studien insgesamt und de wirksamen } \\
\text { Studien, getrennt nach ihren theoretischen } \\
\text { Grundlagen. KBT = kognitiv-behavioral, BT = } \\
\text { verhaltenstherapeutisch, CC = klienten-zentriert, } \\
\text { EDU = edukative Maßnahmen. (nach Ott, 2003, S. } \\
\text { 132) }\end{array}$ & 55 \\
\hline Abb. 9 & 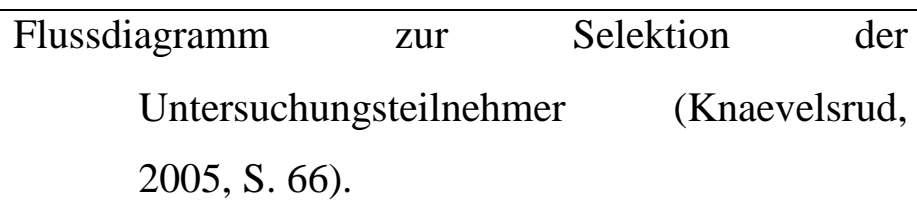 & 58 \\
\hline
\end{tabular}




\begin{tabular}{|c|c|c|}
\hline Abb. 10 & $\begin{array}{l}\text { PTB-Symptome vor Behandlungsbeginn (pre), nach } \\
\text { dem Abschluss der Therapie (post), und in } \\
\text { den beiden Nachuntersuchungen 3Monate } \\
\text { und 1,5Jahre nach Behandlungsabschluss. } \\
\text { Die Linien sthen für verschiedene } \\
\text { Symptome, die mit dem IES-R erfasst } \\
\text { wurden: Intrusionen, Vermeidungsverhalten } \\
\text { (Avoidance) und Überrregbarkeit } \\
\text { (Hyperarousal). (Nach Maercker, 2005, S. } \\
\text { 10). }\end{array}$ & 59 \\
\hline Abb. 11 & $\begin{array}{l}\text { DAS-Werte vor und nach der Therapie getrennt nach } \\
\text { Geschlechtern (Beer \& Breuer, 2003, S. 8) }\end{array}$ & 61 \\
\hline Abb. 12 & $\begin{array}{l}\text { Psychische Beeinträchtigung (SCL-90-R GSI) der } \\
\text { Chat- und Kontrollgruppe (Kordy et al., 2006, S. } \\
\text { 151) }\end{array}$ & 64 \\
\hline
\end{tabular}




\section{Tabellenverzeichnis}

\begin{tabular}{|c|c|c|}
\hline Tabellennummer & Tabellenbeschreibung & Seite \\
\hline Tab. 1 & $\begin{array}{l}\text { Sender-Empfänger-Strukturen } \text { der } \\
\text { Kommunikation im Vergleich (nach Misoch, 2006, S. } \\
\text { 56) }\end{array}$ & 20 \\
\hline Tab. 2 & $\begin{array}{l}\text { Treffer zu den Begriffen „Online-Beratung“ und } \\
\text { „Psychologische Online-Beratung“ in den Internet- } \\
\text { Suchmaschinen Google, Fireball und Yahoo } \\
(20.05 .2007)\end{array}$ & 40 \\
\hline Tab. 3 & $\begin{array}{l}\text { Eigenschaften der Untersuchungsteilnehmer, getrennt } \\
\text { nach Online- und Face-to-face-Gruppe (erstellt nach } \\
\text { den Angaben von Beer \& Breuer, 2003) }\end{array}$ & 60 \\
\hline Tab. 4 & $\begin{array}{l}\text { Demographische Merkmale der Studienteilnehmer } \\
\text { getrennt nach Chat- und Kontrollgruppe (nach } \\
\text { Angaben von Kordy et al., 2006) }\end{array}$ & 62 \\
\hline
\end{tabular}




\section{Einleitung}

Das Internet hat seit seiner Entstehung vor mehr als 30 Jahren immer mehr an Bedeutung in der gesellschaftlichen Kommunikation gewonnen. Damals als Forschungsprojekt des USVerteidigungsministeriums ins Leben gerufen ist es heute fester Bestandteil des Alltags von Millionen Menschen weltweit. Die Zahl der Internetnutzer ist heute über alle Alters- und Bevölkerungsschichten hinweg sehr hoch. Es dient als Präsentationsplattform für Privatpersonen und Unternehmen, als Unterhaltungsmedium, Informationsquelle und Kommunikationsmittel.

Die Rolle, die das Internet im Alltag vieler Menschen spielt, hat es in den vergangen Jahren auch immer mehr in den Blickpunkt der Psychotherapie gerückt. Gemäß der Definition von Strotzka (1974) steht Psychotherapie für einen „bewussten Prozess zur Beeinflussung von Verhaltensstörungen und Leidenszuständen, die in einem Konsensus für behandlungsbedürftig gehalten werden, und zwar mit psychologischen Mitteln (durch Kommunikation: meist verbal, aber auch nonverbal) in Richtung auf ein definiertes, nach Möglichkeit gemeinsam erarbeitetes Ziel mittels lehrbarer Techniken auf der Basis der Theorie des normalen und pathologischen Verhaltens. “

Für die Psychotherapie kann das Internet nicht nur Informations- und Präsentationsgegenstand , sondern auch Forschungsgegenstand sein. Darüber hinaus tauchte die Frage auf, ob die Internetnutzung für psychotherapeutische Intervention möglich ist. Könnte die herkömmliche Kommunikation von Angesicht zu Angesicht durch computervermittelte Kommunikation abgelöst werden oder durch diese ergänzt werden?

Gerade in der Anfangsphase dieser Entwicklung sind in der Fach- wie auch in der Alltagspresse zwei Lager auszumachen, deren Vertreter einander unversöhnlich gegenüber stehen. Glühende Anhänger der internetbasierten Intervention preisen die neuen Konzepte aufgrund ihrer unübersehbaren Vorteile: Ökonomie, Niedrigschwelligkeit und leichte Erreichbarkeit - zu jeder Zeit von jedem Ort. Die Vertreter der Gegenseite führen den Mangel an sozialer und persönlicher Nähe an und weisen auf pathologische Aspekte der Internetnutzung für den therapeutischen Bereich hin.

Ziel der vorliegenden Arbeit ist es, die Skepsis der Psychotherapeuten hinsichtlich des Einsatzes des Internets abzuschwächen. Hierbei werden sowohl die theoretischen Grundlagen als auch die praktische Umsetzung computervermittelter Intervention beleuchtet und eine Übersicht über den Stand der Forschung gegeben. Dabei sollen Vor- und Nachteile des 
Einsatzes neuer Techniken in der therapeutischen Hilfe objektiv dargestellt und diskutiert werden. Ferner wird ein eigens internetbasiertes Beratungsprojekt, sowie erste Erfahrungen mitgeteilt.

\section{Das Internet - Geschichte und Verbreitung}

Um die Bedeutung des Internets für die Psychotherapie und die Einsatzmöglichkeiten im Bereich der psychotherapeutischen Intervention $\mathrm{zu}$ verstehen, muss zunächst auf die Geschichte und die Eigenschaften des Internets eingegangen werden. Die Entstehung des Internets wird daher erläutert und seine Bedeutung für das heutige gesellschaftliche Leben aufgezeigt. Im Anschluss daran werden internetbasierte Kommunikationsformen vorgestellt und die Besonderheiten der Online-Kommunikation diskutiert.

Die folgende Darstellung der Geschichte des Internets ist stark komprimiert, eine ausführlichere Darstellung findet sich beispielsweise bei Hafner \& Lyon (2000).

\subsection{Die Geschichte des Internets}

Das Internet ist eine elektronische Verbindung zwischen verschiedenen Netzwerken von Rechnern, mit dem Ziel des Informations- bzw. Datenaustausches. Dieser Datenaustausch basiert auf einer gemeinsamen „Sprache“, dem Übertragungsprotokoll. Transmission Control Protokoll / Internet Protokoll (kurz: TCP/IP). Dank TCP/IP ist die Kommunikation zwischen Rechnern unabhängig von deren Hardware und Betriebssystem und somit ohne zentrale Vermittlungsstelle möglich. Das war aber nicht immer so.

Die Anfänge des Internets lassen sich zurückverfolgen in die späten 1950er Jahre: Der Start des sowjetischen Erdsatelliten Sputnik am 4. Oktober 1957 stellt den Überlegenheitsanspruch der westlichen Welt in Frage und löst insbesondere in den USA große Bestürzung aus. Dieser so genannte Sputnik-Schock ist für die US-amerikanische Regierung unter Präsident Dwight D. Eisenhower Anlass, den Forschungsetat zu erhöhen und neue Institutionen ins Leben zu rufen, die die amerikanische Forschung wieder in eine weltweite Führungsposition bringen sollen. Im Zuge dieser Bemühungen wird im Januar 1958 auch die advanced research projects agency (ARPA) gegründet, die dem Verteidigungsministerium untergeordnet ist und die Aufgabe hat, neuartige Technologien zu entwickeln.

Vier Jahre nach der Gründung der ARPA wird der Psychologe Joseph Carl Robnett Licklider eingeladen, die Abteilung „Command and Control“ sowie das neugeschaffene Information 
Processing Techniques Office (IPTO) zu leiten. Licklider hat kurz zuvor durch die Veröffentlichung einer Abhandlung zum Thema „Man-Computer Symbioses“ (Licklider, 1960) auf sich aufmerksam gemacht und scheint daher für diesen Posten wie geschaffen. Während seiner Amtszeit kann Licklider zwei Entwicklungen vorantreiben, die sich rückblickend als Meilensteile auf dem Weg zur Entstehung des Internets erweisen. Durch die Erforschung und Ausarbeitung des Time-Sharing-Konzeptes (mehrere Benutzer konnten gleichzeitig an einem Rechner arbeiten und teilten sich somit die Rechenzeit des Computers) wird es möglich, einen Zentralrechner über verschiedene Terminals zu nutzen. Das erste Computernetzwerk ist installiert. Zum anderen wird eine neue Dimension der MenschMaschine-Interaktion erreicht: an den Terminals ist der Einsatz von Lochkarten oder Magnetbändern überflüssig; der Operator kann über das Terminal direkt mit dem Rechner interagieren. Die Netzwerk-Konzeption auf Basis des Time-Sharing-Konzeptes birgt aber auch Nachteile: einerseits handelt es sich um zentrale Netzwerke (vgl. Abb.1), die ein zentrales Terminal zur Vermittlung benötigen, andererseits ist die Kommunikation nur zwischen solchen Rechnern möglich, die vom selben Hersteller und Typ sind.

Die Vision Lickliders ist es schon zu dieser Zeit, den Datenaustausch unabhängig von der Hardware des jeweiligen Nutzers zu machen und eine übergreifende „Sprache“, ein einheitliches Übertragungsprotokoll einzuführen. Diese Vision wird Jahre später mit dem TCP / IP (ein universelles Protokoll, dass es erlaubt nahezu alle Betriebssysteme von Computern zu vernetzen) Realität werden.

\footnotetext{
„Man stelle sich folgende Situation vor: mehrere verschiedene Zentren werden vernetzt, doch jedes verhält sich höchst individualistisch und besitzt seine eigene spezielle Sprache und Verfahrensweise. Ist es nicht wünschenswert, ja sogar notwendig, dass sich alle Zentren auf eine Sprache einigen oder zumindest auf einige Konventionen, nach denen Fragen gestellt werden können wie ,Welche Sprache sprichst Du?'“
}

(Licklider, zitiert nach Hafner \& Lyon, 2000, S. 44).

Unabhängig vom Forschungsbetrieb der ARPA untersucht Paul Baran, ein Wissenschaftler der RAND Corporation, im Auftrag der U.S. Air Force die Angreifbarkeit von Netzwerken. Er unterscheidet verschiedene Netzwerkstrukturen (vgl. Abb.1) und beschreibt das „Distributed Network“ in seiner 1964 veröffentlichten gleichnamigen Untersuchung als dem höchst angreifbaren zentralisierten Netzwerk deutlich überlegen. Die Datenübertragung in einem solchen Netzwerk sollte paketorientiert sein, wobei sich die (Daten-)Pakete die jeweils 
beste zur Verfügung stehende Route aussuchen. Diese Idee wird auch von Leonard Kleinrock (1961) propagiert, der einen weiteren Vorteil in der Ökonomie der paketorientierten Übertragung sieht: beim eventuellen Verlust oder einer Beschädigung einzelner Pakete während des Transfers, müssen nur die entsprechenden Pakete, nicht aber die gesamte Datei erneut übertragen werden.

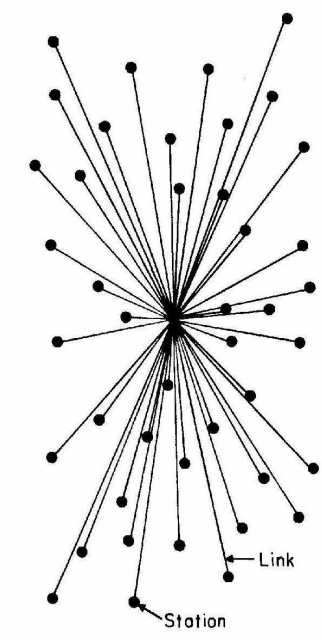

CENTRALIZED
(A)

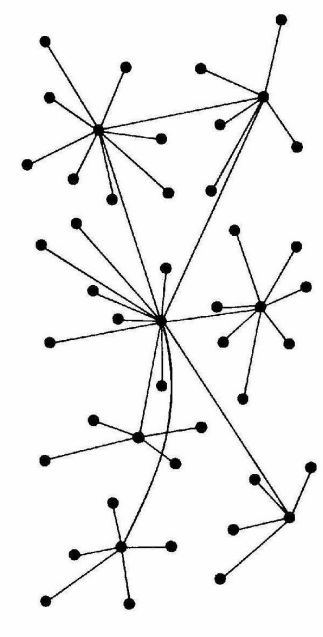

DECENTRALIZED
(B)

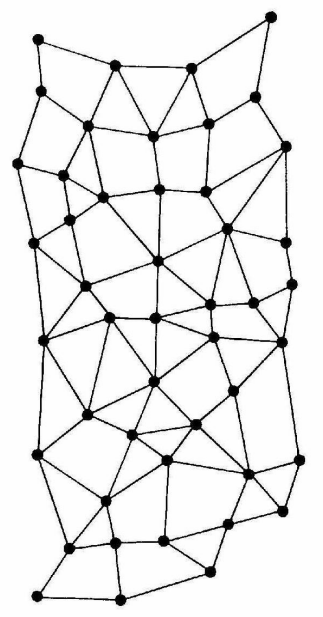

DISTRIBUTED

Abb. 1: Netzwerkstrukturen (nach Baran, 1964, S. 2)

Bob Taylor, ein Nachfolger Lickliders, ruft schließlich ein Projekt ins Leben, das das Ziel verfolgt, einen neuartigen Computerverband zu entwickeln. Die Idee kommt Taylor durch die Vielzahl von Terminals, die in seinem Büro stehen und notwendig sind, um auf die Rechenzentren der dem Programm angeschlossenen Universitäten zugreifen zu können. Das neu entwickelte Computernetzwerk verbindet zunächst vier Standorte miteinander: die University of Utah (Salt Lake City), die UCLA (University of California, Los Angeles), die University of California (Santa Barbara) sowie das Stanford Research Institute (Menlo Park). Im Laufe der Zeit soll der Verband aber weiter ausgebaut werden.

In der Anfangsphase des Computernetzwerkes besteht allerdings weiterhin das Problem, dass die verschiedenen Systeme unterschiedliche „Sprachen“ sprechen und Netzwerkprogramme zur Kommunikation zwischen den Systemen daher für jeden Rechnertyp neu geschrieben oder angepasst werden müssen. Es soll daher ein Programm entwickelt werden, dass als eine Art „Dolmetscher“ zwischen den verschiedenen Computer-Sprachen fungiert: ein so genannter Interface Message Processor (IMP). Auch die Datenübertragung gestaltet sich anfangs problematisch. Die fünf Jahre zuvor veröffentlichte Arbeit von Paul Baran erweist sich hier als äußerst hilfreich. Ohne seine direkte Beteiligung werden Barans Ideen unter 
Leitung von Len Kleinrock umgesetzt. Ende des Jahres 1969 findet der erste Testlauf des ARPAnets statt. Bis zum Internet in seiner heutigen Form ist es aber noch ein weiter Weg.

Ein erster Schritt auf diesem Weg wird im Jahr 1972 mit der Entwicklung eines Programms zum Versenden und Empfangen von E-Mails gemacht. Im selben Jahr wird das ARPAnet auf einer internationalen Computerkonferenz vorgestellt und gewinnt damit deutlich an Popularität. Andere Netzwerke werden nach dem Vorbild des ARPAnets geknüpft und unterschiedliche Übertragungsmöglichkeiten - mittels Radiowellen oder per Satellit statt via Telefonleitung - werden getestet. So wird aus dem ursprünglich kleinen Computerverbund ein Netzwerk von Netzwerken.

Eine weitere bahnbrechende Entwicklung wird im Jahr 1974 gemacht: das Transmission Control Protocol (TCP). Später wird TCP weiterentwickelt werden zum Transmission Control Protocol / Internet Protocol (TCP/IP). Mit Hilfe dieser Technologie können Daten sehr einfach zerteilt und wieder zusammengesetzt werden, was eine wichtige Voraussetzung für den paketvermittelten Transfer ist, wie Baran und Kleinrock ihn beschreiben. Mit TCP/IP ist endlich die übergreifende Sprache gefunden, in der Computer verschiedenen Typs und unterschiedlicher Hersteller miteinander kommunizieren können. Dem Siegeszug des Internets steht nur noch eines im Weg: die Entwicklung der Computersoftware ist der Netzwerktechnik weit unterlegen. Bislang fehlt es noch an einem leistungsfähigen und stabilen Betriebssystem, mit dem Computer das Netzwerk effektiv nutzen können.

Als Lösung wurde hier das UNIX-System (ein Mehrbenutzerbetriebssystem) herangezogen, das zwar bereits im Jahr 1968 von Ken Thompson entwickelt worden war, aber erst seit 1974 mit der Veröffentlichung des Source Codes öffentliche Anerkennung findet. Das System verbreitet sich von da an schnell und zahlreiche Studenten nutzen die Chance, das Programm $\mathrm{zu}$ verbessern und weiterzuentwickeln. Aus diesem Grund wird im Jahr 1978, als das ursprüngliche UNIX-System kommerzialisiert wird, von der Universität Berkeley weiterhin eine freie Version (BSD) angeboten. BSD konnte sich durch das enorme EntwicklerPotential, auf das Open Source-Programme zurückgreifen können, ständig weiterentwickeln und ist auch die Grundlage heutiger UNIX-Versionen.

Ein Bestandteil von BSD ist UUCP (UNIX to UNIX Copy Protocol), eine Art virtuellen schwarzen Bretts, auf dem Nachrichten hinterlassen und von anderen Nutzern an anderen Standorten eingesehen werden können. UUC ist der Vorläufer moderner Internetforen und wird im Jahr 1981 in Berkeley mit dem ARPAnet verknüpft.

Ungefähr zur gleichen Zeit eröffnen Roy Trubshaw und Richard Bartle an der Universität von Essex das erste „Multi User Dungeon“ (MUD). (Dieses MUD macht es möglich, dass 
mehrere Spieler gleichzeitig und interaktiv ein textbasiertes Abenteuerspiel spielen). Das Multi User Dungeon ist das erste Programm, das nicht zur wissenschaftlichen Nutzung entwickelt wurde, sondern ausschließlich zur Unterhaltung der Nutzer dient. Auch heute noch werden MUDs wegen ihrer komplexen Darstellung einer virtuellen Realität der Vielzahl an Interaktionsmöglichkeiten, die sich den Teilnehmern bieten, geschätzt.

Obwohl mit dem MUD bereits der erste Schritt in Richtung Unterhaltungsmedium gemacht ist, wird das ARPAnet vorerst weiter lediglich zu wissenschaftlichen Zwecken verwendet. Das ursprünglich zur militärischen Forschung entwickelte Netzwerk wird aber um Netzwerke erweitert, die andere thematische Schwerpunkte setzen: so etwa das Computer Science Network CSNet, das im Jahr 1979 gegründet wird.

Anfang der 1980er werden die ersten Netzwerke in Europa gegründet, zum ersten Mal verlässt die neue Netzwerktechnik das us-amerikanische Territorium. Im gleichen Zeitraum forciert das amerikanische Verteidigungsministerium die Umstellung aller Netzwerke auf TCP/IP. Trotz der mittlerweile sehr großen Reichweite der Netzwerke und der entstandenen Kommunikations- und Unterhaltungsmöglichkeiten wird der Zugang weiterhin auf militärische oder wissenschaftliche Institutionen beschränkt, die kommerzielle Nutzung des Netzwerks ist verboten. Erst im Jahr 1991 wird diese Blockade aufgehoben und somit das eigentliche Internet ermöglicht.

Das Netzwerk ist aber weiterhin nur für eine geringe Zahl von Nutzern attraktiv, da die Nutzung per Kommandozeile erfolgt und sich daher nur Fachleuten erschließt. Fast zeitgleich werden zwei Programme entwickelt, die es erlauben, das Internet über eine grafische Benutzeroberfläche zu bedienen: Gopher und WorldWideWeb. Da die Universität von Minnesota Lizenzgebühren für Gopher einführt, kann sich dieses System nicht durchsetzen.

Das World Wide Web (WWW) wird von einem Informatiker am Genfer Institut für Teilchenphysik, Tim Berners-Lee, entwickelt und ist ursprünglich als Intranet intendiert. Dieses Programm kann Hypertextseiten erstellen und anzeigen, benötigt aber ein weiteres Zusatztool, einen so genannten Browser, der das Hypertext Transfer Protokoll interpretieren und darstellen kann. Der erste Browser wird 1992 von einem Studenten der Universität Berkeley entwickelt und heißt ViolaWWW. Dieser Browser dient als Vorbild für weitere Entwicklungen und ist unseren heutigen Browsern sehr ähnlich.

Im darauf folgenden Jahr beginnt das Genfer Institut für Teilchenphysik mit der Computerforschungsabteilung der Universität Illionois (National Center for Supercomputing Applications) zu kooperieren und bald darauf werden die ersten Browser für Windows und Mac veröffentlicht. Von diesem Zeitpunkt an ist die Verbreitung der neuen Technologie nicht 
mehr zu bremsen. Bereits im Herbst 1993 gibt es mehr als 500 bekannte WorldWideWebServer und die Wachstumsrate des WWW-Dienstes liegt bei über $300.000 \%$.

Heute werden die Begriffe WorldWideWeb und Internet meist synonym verwendet. Die Datenübertragung erfolgt nach wie vor mittels TCP/IP, geändert hat sich lediglich die Geschwindigkeit, mit der die Daten übermittelt werden. Mit anderen Diensten ist es möglich, auf Dateisysteme von Computern zuzugreifen und diese auszutauschen.

\subsection{Internetnutzung heute}

Wie sich aus der historischen Entwicklung des Internets erschließt, handelt es sich hierbei noch um ein relativ junges Medium, das erst seit ca. 15 Jahren für die breite Masse zugänglich ist, nachdem es zuvor ausschließlich dem wissenschaftlichen und militärischen Betrieb vorbehalten war. In der gleichen Zeit hat sich auch das Online-Angebot immer weiter ausgedehnt und verfeinert. So wurde das Internet für immer mehr Menschen interessant. Es dient längst nicht mehr nur wissenschaftlichen, militärischen und kommerziellen Zwecken; das Internet ist zum heutigen Zeitpunkt auch Unterhaltungsmedium und Kommunikationsmittel und in unserer Gesellschaft fast ebenso verbreitet wie die klassischen technischen Massenmedien Rundfunk und Fernsehen. Das Internet ist aus dem Alltag der Menschen wie aus dem wissenschaftlichen Betrieb heute nicht mehr wegzudenken.

Abbildung 2 zeigt den rasanten Anstieg der Anzahl an Internetnutzern weltweit seit der Jahrtausendwende. Anhand dieser Zahlen liegt die Vermutung nahe, dass das Medium Internet in allen Teilen der Welt weit verbreitet ist.

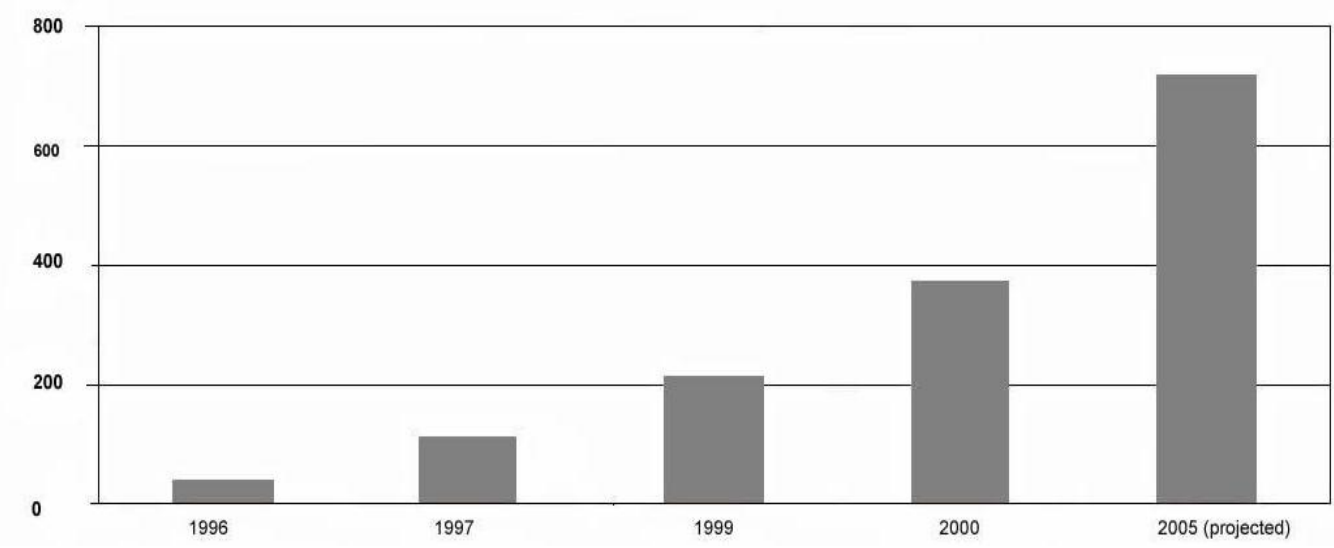

Abb. 2: Internetnutzer weltweit in Millionen (nach Brym \& Lenton, 2001, S. 6) 
Bei genauerer Betrachtung der Nutzungsdaten kann man jedoch feststellen, dass die Internetnutzung keineswegs über die Länder hinweg gleich ausfällt. Im Gegenteil lässt sich eindeutlicher „digital divide“ verzeichnen (vgl. hierzu Wresch, 1996; Bolt \& Crawford, 2000; Norris, 2002; Chen \& Wellman, 2003; Gehrke, 2004). Dieser divide geht zu Ungunsten der ärmeren, wenig industrialisierten Länder (Eine detaillierte Tabelle zur weltweiten Internetnutzung findet sich im Anhang). Nach den Angaben der Internetworldstats.com (Stand März 2007) haben den größten Anteil an der weltweiten Internetnutzung Asien $(35,8 \%)$, Europa $(28,3 \%)$ und Nordamerika $(20,9 \%)$.

Diese Zahlen allein sind aber noch wenig aussagekräftig, von wesentlich größerer Bedeutung ist der Anteil der Bevölkerung einer Region, der Zugang zum Internet hat. Auch hier liegen Nordamerika $(69,7 \%)$ und Europa $(38,9 \%)$ auf den vorderen Plätzen, zusammen mit Australien (53,5\%). Kaum Zugang zum Internet haben dagegen die Menschen im Mittleren Osten $(10,0 \%)$ und in Afrika (3,6\%).

Da es sich beim Internet aber um ein noch relativ neues Medium handelt, bleibt die Hoffnung, dass sich diese Ungleichverteilung der Internetnutzung in den kommenden Jahren und Jahrzehnten aufheben wird. Ein Blick auf die Wachstumsraten (s. Anhang 1) der einzelnen Regionen zeigt, dass diese Hoffnung berechtigt ist: insbesondere in den bislang benachteiligten Ländern sind in den letzten sieben Jahren sehr hohe Wachstumsraten zu verzeichnen. Es kann also davon ausgegangen werden, dass der „digital divide“ ein rückläufiges Phänomen ist. Zwei der führenden Forscher auf diesem Gebiet, Paul DiMaggio und Eszter Hargittai (z. B. DiMaggio \& Hargittai, 2001; Hargittai, 2002) vertreten die Auffassung, dass eine neue Ungleichverteilung die alte ablöst: an die Stelle des „digital divides“, der heterogenen Internetnutzung im internationalen Vergleich tritt das Phänomen der „digital inequality“, der ungleichen Nutzungschancen innerhalb eines Landes. DiMaggio \& Hargittai sind der Ansicht, dass der physische Zugang zum Internet aktuell kaum jemandem verwehrt bleibt, die Menschen aber über ungleiche Nutzungsmöglichkeiten verfügen, was durch fünf Faktoren bedingt wird: technische Ausstattung, Autonomie der Nutzung, technische Fähigkeiten, soziale Unterstützung bzw. Anleitung durch erfahrene Benutzer sowie der Zweck der Internetnutzung.

Diese Faktoren werden hier bei der Betrachtung der Internetverbreitung in Deutschland nicht berücksichtigt; es soll lediglich die Internetnutzung selbst betrachtet werden, Nutzungsfähigkeiten bleiben unberücksichtigt. 


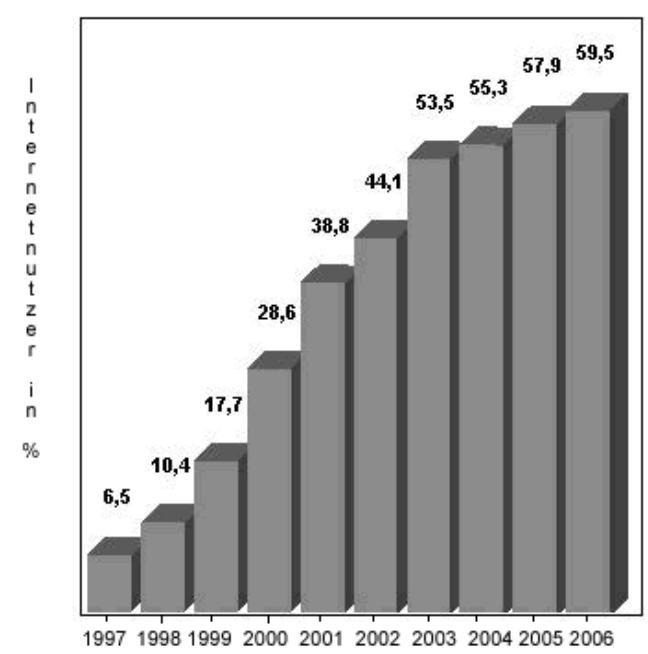

Abb.3: Entwicklung der Internetnutzung in Deutschland von 1997 bis 2006 (nach den Ergebnissen von Eimeren \& Frees, 2006)

Der Anteil der deutschen Bevölkerung, der das Internet nutzt, ist in den vergangenen zehn Jahren drastisch angestiegen (vgl. Abb. 3): hatten im Jahr 1997 noch lediglich 6,5\% der Deutschen Zugang zum Internet, so sind es im Jahr 2006 knapp 60\%. Im internationalen Vergleich liegt Deutschland damit weit vorn (s. Anhang 1). Eine Untersuchung des Marktforschungsinstituts Fittkau \& Maaß (2005) zeigt, dass das Internet nicht nur von vielen Menschen genutzt wird, sondern auch eine große Bedeutung für die Nutzer hat. Von den Befragten sagten 39,1\%, dass das Internet „eine wichtige Rolle“ in ihrem Leben spiele, 42,3\% gaben sogar an, ,,auf keinen Fall (darauf) verzichten“ zu wollen (vgl. Fittkau\& Maaß, 2005).

Eine andere Untersuchung desselben Unternehmens hatte die Bedeutung unterschiedlicher Kommunikationsmethoden zum Inhalt (Fittkau \& Maaß, 2004). Befragt wurden hierzu Nutzer der Internetplattform OpenBC aus Europa; die Umfrage fand online statt und wurde auf deutsch, englisch, französisch und spanisch durchgeführt. Die Teilnehmer sollten die subjektiv eingeschätzte Wichtigkeit verschiedener Kommunikationsformen für die Kontaktpflege angeben. Das Ergebnis war durchaus unerwartet: mehr als 95\% der befragten schätzten E-Mail und Messenger-Kontakte als „wichtig“ oder „sehr wichtig“ ein (s. Abb. 4), von telefonischen Kontakten sagten dies nur ca. $3 \%$ weniger. 
Wichtigkeit von Kommunikationsformen und Orten im Vergleich

Basis: openBC.com-Nutzer, "sehr wichtig« und wwichtig"

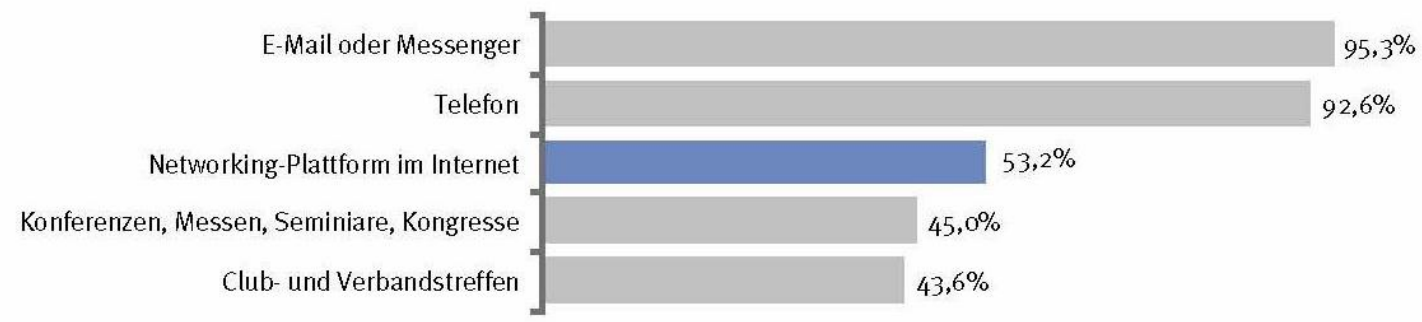

Abb. 4: Wichtigkeit von Kommunikationsformen im Vergleich, Prozentzahlen geben an, welcher Anteil der Befragten die jeweilige Kommunikationsform als „wichtig“ oder sehr wichtig“ eingestuft haben (nach Fittkau \& Maaß, 2004)

Der Online-Kommunikation wird also von den Befragten ein größerer Stellenwert eingeräumt als den herkömmlichen Kommunikationsmethoden.

\subsection{Kommunikationsmöglichkeiten via Internet}

Das Internet bietet zahlreiche Möglichkeiten, mit anderen Menschen in Kontakt zu treten. (Im Anhang befinden sich Beispiele für einige der hier beschriebenen computervermittelten Kommunikationsformen, die Essstörungen zum Thema haben).Hierbei muss zwischen verschiedenen Diensten und Anwendungen der jeweiligen Dienste unterschieden werden. Dienste verfügen über ein eigenes Datenübertragungsprotokoll, welches den Datenfluss zwischen Rechnern regelt. Die wichtigsten Dienste des Internets, die zur Kommunikation genutzt werden können sind:

\section{$\S \quad$ World Wide Web (WWW)}

Wie bereits oben erwähnt, wurde das World Wide Web 1989 von Tim Berners Lee am CERN in Genf entwickelt, wo es eigentlich nur zum institutsinternen Datenaustausch verwendet werden sollt. Heute ist das WWW der wohl bekannteste Dienst des Internets. Der Datenaustausch im WWW wird über ein Hypertextprotokoll (Hypertexttransferprotocol HTTP) gesteuert. Die Bedienung ist für den Nutzer dank eines Zusatzprogramms (Browser) mit grafischer Oberfläche besonders einfach. Das WWW stellt Art Kombination anderer Internetdienste wie etwa FTP, Telnet und E-Mail unter einer grafischen Benutzeroberfläche dar und ermöglicht somit den Austausch multimedialer Daten (vgl. hierzu Musch, 1997). 


\section{$\S \quad$ E-Mail}

Die E-Mail (von engl. electronic mail; dt.: „die elektronische Post“) ist eine briefartige Nachricht, die auf elektronischem Wege in Computernetzwerken übertragen wird. Das Übertragungsprotokoll in diesem Fall heißt SMTP (Simple Mail Transfer Protocol). Die auf dem Mailserver eingegangenen Nachrichten werden mit Hilfe eines weiteren Programms (z.B. POP3) auf den eigenen Rechner geladen, wo sie mit einem gesonderten Mailprogramm gelesen und bearbeitet werden können. Der Versand von E-Mails ist aber auch ohne Zusatzsoftware über WWW möglich.

Eine E-Mail hat - wie auch ein Brief - einen bestimmten Absender und einen (oder mehrere) Empfänger. Die Adresse setzt sich zusammen aus dem Namen (oder Pseudonym) des Nutzers, dem Hostnamen und der Länderkennung. Die Adresse max.muster@ruhr-uni-bochum.de bedeutet beispielsweise dass Max Muster seinen EMail-Account (E-Mail-Zugang; virtuelles Postfach) an der Universität Bochum hat. Neben Textbotschaften können mit einer E-Mail auch Audio-, Video- oder andere Dateien verschickt werden.

Der Austausch von E-Mails ist ähnlich wie der Versand von Briefen eine Form der asynchronen Kommunikation, allerdings mit wesentlich kürzerer Übertragungszeit.

\section{$\S$ Newsgroup / Usenet}

Newsgroups sind virtuelle Diskussionsforen im Internet. Die Nutzer können hier Nachrichten (Postings) auf einem Newsserver hinterlassen, wo andere Nutzer sie einsehen und darauf reagieren können, wobei die Beiträge zu einem Thema als Thread oder Topic zusammengefasst werden. Es handelt sich also um eine asynchrone Kommunikationsform zwischen mehreren Personen. Meist gibt der Name der Newsgroup Aufschluss über den thematischen Schwerpunkt und die Sprache, in der die Beiträge verfasst werden. Die deutsche wissenschaftliche Newsgroup zum Thema Psychologie hat beispielsweise den Namen „de.sci.psychologie“, wobei „de“ für deutsch und „sci“ für scientific steht. Es gibt aber nicht nur wissenschaftliche Diskussionsgruppen zu verschiedenen Fachbereichen, sondern auch Gesprächsgruppen zu Alttagsthemen und Selbsthilfegruppen, wie zum Beispiel de.etc.selbsthilfe.angst: ein deutsches Selbsthilfeforum für Menschen mit einer Angststörung und deren Angehörige.

Als Übertragungsprotokoll bei Newsgroups wird meist NNTP (Net News Transfer Protocol) verwendet. Die Beiträge können aber auch über einen WWW-Browser eingesehen und verfasst werden (vgl. Webforum). Eine weitere Form der Newsgroup sind 
so genannte Mailinglisten, bei denen die Postings nicht nur auf dem Newsserver gespeichert, sondern auch automatisch per E-Mail an alle registrierten Nutzer verschickt werden.

Das Usenet ist ein äußerst bekanntes Newssystem, das zahlreiche Newsserver weltweit miteinander verbindet.

\section{$\S \quad$ IRC (Internet Relay Chat)}

Chatten (von engl. to chat - plaudern) ist eine Form der synchronen Kommunikation, bei der sich mehrere Nutzer via Textbotschaften miteinander, unterhalten' können. Jeder, der sich bei einem IRC-Server angemeldet hat, kann an dieser Kommunikation teilnehmen.

Entwickelt wurde der Internet Relay Chat von einem finnischen Studenten namens Jarkko Oikarinen im Jahr 1989. Obwohl der Entwickler diese Methode ursprünglich ausschließlich zum privaten Gebrauch gedacht hatte, ist Chatten mittlerweile die "wohl populärste Form der Online-Kommunikation" (Filinski 1998, 23).

Neben dem Internet Relay Chat gibt es noch zwei weitere Formen des Chattens (vgl Runkehl et al. 1998): Web-Chats - eine Anwendung des World Wide Web und OnlineChats, die von Providern mit spezieller Software betrieben werden.

Wer an einem Chat teilnehmen möchte, muss sich mit einem Pseudonym, einem sog. Nickname, anmelden und betritt daraufhin einen virtuellen Raum. Die übermittelten Textbotschaften sind von allen eingeloggten Benutzern einsehbar. Somit handelt es sich um eine Kommunikationsform, bei der sich viele mit vielen austauschen. Es gibt neben diesen öffentlichen Chaträumen gibt es auch die Möglichkeit zu ,flüstern', so dass die Nachricht nur von einem bestimmten Adressaten eingesehen werden kann, sowie virtuelle Separees, die nur mit Passwort betreten werden können. Die beim Chatten ausgetauschten Nachrichten werden nicht zentral gespeichert, wie dies bei Newsgroups der Fall ist, sondern allenfalls lokal auf den Festplatten der Nutzer.

\section{$\S \quad$ MUD (Multiple User Dungeon)}

Der erste Multiple User Dungeon (MUD) wurde Anfang der 1980er von Roy Trubshaw und Richard Bartle an der Universität von Essex entwickelt. Bei MUDs handelt es sich um Online-Rollenspiele, die auf einem Server bereitgestellt werden. Die Übertragung erfolgt mittels Telnet. Die Spiele verfügen nicht über eine grafische Oberfläche, sondern sind textbasiert und können von mehreren Spielern gleichzeitig gespielt werden. Der Spieler tritt im MUD in Form eines Avatars, eines von ihm gestalteten Charakters in 
Erscheinung. Meist handelt es sich um Fantasy- oder Abenteuerspiele. Zusätzlich zu den Spielfunktionen können die Teilnehmer auch miteinander kommunizieren.

\section{$\S \quad$ Voice over IP (VOIP)}

Mit dem Begriff bezeichnet man das Telefonieren über Computernetzwerke, die nach Internet-Standards aufgebaut sind. Andere gebräuchliche Bezeichnungen sind InternetTelefonie bzw. IP-Telefonie (Internet Protokoll-Telefonie). Zum Telefonieren via IP können Computer und Headset, spezielle IP-Telefonie-Geräte oder herkömmliche Telefone verwendet werden, sofern diese mit einem Adapter ausgestattet werden. Die Nutzung unterscheidet sich dann nicht von herkömmlicher Telefonie.

Das World Wide Web ist wohl der bekannteste Online-Dienst, weswegen die Begriffe World Wide Web und Internet umgangssprachlich synonym verwendet werden. Mit Hilfe spezieller Software können Anwendungen des World Wide Web genutzt werden. Hier sind zu nennen:

\section{$\S \quad$ IM (Instant Messaging)}

Instant Messaging ist eine Form der synchronen Kommunikation. Mit Hilfe einer speziellen Software (Client) können hier in Echtzeit mit einem anderen User Informationen ausgetauscht werden. Es handelt sich hierbei üblicherweise um eine Form der Textkommunikation ohne Bild- und Tonübertragung. Die meisten Instant-Messenger verwalten Kontaktlisten und zeigen dem Nutzer, welche anderen Personen präsent sind. Wer einen Instant Messenger nutzen möchte, muss sich registrieren, wobei ihm eine Nutzernummer zugeordnet wird. Dann kann eine Freundesliste mit bestimmten Suchkriterien angelegt werden. Beim Programmstart wird dem Teilnehmer mitgeteilt, welche weiteren Nutzer aus seiner Freundesliste anwesend sind. Mit diesen Personen kann der Nutzer dann Nachrichten austauschen, die nicht für Dritte einsichtig sind.

\section{$\S$ Web Chat}

Ein Web-Chat ist ein Dienst des World Wide Web, der in seinen Nutzungsfunktionen mit dem Internet Relay Chat vergleichbar ist. Er unterscheidet sich von diesem nur dadurch, dass der Zugriff nicht über einen IRC-Server erfolgt, sondern mit Hilfe des normalen Webbrowsers. 


\section{$\S$ Webforen}

Webforen sind ebenfalls eine Anwendung des World Wide Webs, der Zugriff auf diese virtuellen Diskussionsplattformen erfolgt über eine Internetseite. Man unterscheidet zwischen offenen Foren, an denen alle Internetnutzer uneingeschränkt teilnehmen können und solchen, die eine Registrierung voraussetzen. Die Nutzer solcher geschlossener Foren verstehen sich meist als eine Art Gemeinschaft (Community). Webforen bieten die gleichen Funktionen wie andere Internetforen auch, bieten darüber hinaus aber häufig noch weitere Optionen, wie etwa Kalender mit den Geburtstagen der registrierten User oder das Signieren der Postings durch kleine Benutzerbilder. Diese Zusatzfunktionen dienen der Herausbildung einer Online-Identität und der Verstärkung des Gemeinschaftsgefühls der Online-Community.

\section{$\S$ Homepages}

Im Internet können Personen sich eine Domain sichern, d. h. eine Internetseite mit eigener URL. Auf solchen Internetseiten kann der Betreiber dann Informationen in Text- und Bildform sowie Multimedia-Dateien veröffentlichen.

Private Homepages dienen häufig der Vorstellung der eigenen Personen. Über interaktive Elemente wie ein Gästebuch können die Besucher der Webseite dem Betreiber Nachrichten an den Betreiber oder andere Besucher auf der Homepage hinterlassen. Neben privaten Homepages mit eigener URL bieten auch Web-Chats und so genannte facebooks ihren Nutzern die Möglichkeit, sich auf einer eigenen Seite den anderen Teilnehmern vorzustellen. Bei Facebooks handelt es sich um kostenslose Internetplattformen, die der Bildung sozialer Netzwerke dienen sollen, die sich meist an eine bestimmte Zielgruppe (Teenager, Studenten, Manager etc.) wenden. Die Profilseiten solcher face-books sind quasi eigene kleine Homepages, wobei die Seiten der Nutzer untereinander vernetzt sind, um das Kennenlernen zu erleichtern.

\section{$\S$ Weblogs}

Das Wort Weblog setzt sich aus den englischen Begriffen Web (Gewebe, Netz; hier stellvertretend für World Wide Web) und log (Logbuch) zusammen und bezeichnet eine Webseite, auf der regelmäßig neue Beiträge erstellt werden. Es handelt sich bei diesen Weblogs (kurz: Blogs) also um eine Art virtuelle Tagebücher, die sich einem bestimmten 
Thema widmen oder allgemeine Beiträge enthalten können. Sie können von einzelnen Personen oder Gruppen geleitet, privater oder kommerzieller Natur sein. Der Hauptunterschied zu herkömmlichen Homepages besteht darin, dass Blogs regelmäßig durch neue Beiträge erweitert werden, wobei die alten Inhalte nicht gelöscht oder ersetzt werden, sondern lediglich auf der Seite nach unten rutschen.

\section{Computervermittelte Kommunikation}

\subsection{Eigenschaften computervermittelter Kommunikation}

Computervermittelte Kommunikation besitzt bestimmte Eigenschaften, die sie von anderen Formen der Kommunikation unterschieden. Am besten erschließen sich diese Charakteristika computervermittelter Kommunikation im Vergleich mit der Face-to-Face-Kommunikation. Eine ausführliche Darstellung findet sich u. a. bei Misoch (2006, S.54 ff.). Ein solcher Vergleich ist besonders für die Psychotherapie wichtig.

\subsubsection{Synchronität und Asynchronität}

Im Gegensatz zur Face-to-Face-Kommunikation ist computervermittelte Verständigung aber nicht immer synchron, sondern kann auch zeitversetzt erfolgen. Das Chatten beispielsweise ist eine computervermittelte Kommunikationsform, bei der es mehrere Sender und mehrere Empfänger gibt, der Austausch aber in Echtzeit, also synchron erfolgt. Die Verständigung via E-Mail dagegen ist eine asynchrone Kommunikationsform, bei der es einen Sender und in aller Regel auch nur einen Empfänger gibt. Tabelle 1 zeigt einen Überblick über die Formen computervermittelter Kommunikation, geordnet nach Synchronität und Sender-EmpfängerStruktur.

Tab. 1: Sender-Empfänger-Strukturen der Online-Kommunikation im Vergleich (nach Misoch, 2006, S. 56)

\begin{tabular}{l|l|l|l}
\hline & One-to-one & One-to-many & Many-to-many \\
\hline asynchron & E-Mail & WWW & Mailinglist \\
& & Weblog & Newsgroup \\
& & Fomepage & Forum \\
Weblog (kollektiv) & Chat \\
& & MUD \\
\hline synchron & Chat & Seiten &
\end{tabular}




$\left|\begin{array}{l}\text { VoIP (Internet- } \\ \text { Telefonie) }\end{array}\right|$

\subsubsection{Entkörperlichung und Anonymität}

Ein weiterer wesentlicher Unterschied zwischen computervermittelter und Face-to-FaceKommunikation ist darin zu sehen, dass bei den meisten - textbasierten - Formen der computervermittelten Kommunikation die Gesprächspartner einander nicht sehen können. Anders als bei der Verständigung zwischen zwei (oder mehreren) kopräsenten Personen, können bei der Kommunikation via Internet nur textuelle Informationen ausgetauscht und bei der Eindrucksbildung und Interpretation berücksichtigt werden. In sozialen Situationen dagegen fließen außer den gesprochenen Botschaften auch nonverbale Informationen wie Körperhaltung und Gesichtsausdruck in die Bewertung des gesagten mit ein. Der Eindruck, den man sich von seinem Gesprächspartner bildet wird darüber hinaus von dessen Aussehen, Kleidung und den impliziten Informationen über sozialen Status und Haltung, die sich anhand dieser Eindrücke erschließen lassen, beeinflusst. Studien zur Eindrucksbildung zeigen, dass den nonverbalen Kommunikationsinhalten sogar mehr Gewicht eingeräumt wird als dem gesprochenen Wort. Bei der computervermittelten Kommunikation ist man auf die Informationen angewiesen, die das Gegenüber freiwillig gibt, der Fokus rückt automatisch mangels zusätzlicher nonverbaler Information - auf den Inhalt des Gesagten bzw. Geschriebenen. Wenn man den Gesprächspartner nicht auch aus der realen Welt kennt, können selbst Informationen wie Alter und Geschlecht nicht überprüft werden. Bei computervermittelter Kommunikation sind die Gesprächspartner also vollkommen anonym und sind somit in der Lage, sich eine neue virtuelle Identität zuzulegen, eine so genannte Pseudonymität. Das Phänomen der Pseudonymität wird kontrovers diskutiert, da es einerseits therapeutischen Nutzen haben kann (Turkle, 1998), andererseits aber auch zu kriminellen Zwecken missbraucht werden kann. Insbesondere der letzte Aspekt der Pseudonymität ist in jüngster Vergangenheit in den Blickpunkt der Medien gerückt (vgl. z. B. Demmer \& Ludwig, 2006). Insgesamt ist dieser Aspekt somit von erheblicher Bedeutung für die Psychotherapie.

\subsubsection{Textualität}

Die meisten Formen der computervermittelten Kommunikation sind textbasiert. Zwar gibt es dank VoIP auch die Möglichkeit der Sprachübertragung und auch eine Übertragung visueller Informationen mittels Webcam ist möglich, allerdings ist diese oft von unzureichender Qualität. Bei der Kommunikation via E-Mail oder Instant Messaging ist eine Übertragung von 
Bild- und Toniformationen nicht vorgesehen. Somit setzt computervermittelte Kommunikation voraus, dass die Gesprächspartner des Lesens und Schreibens mächtig sind. Andererseits beinhaltet diese Textgebundenheit computervermittelter Kommunikation, dass Gefühle bzw. gefühlsmäßige Reaktionen nicht direkt gezeigt, sondern nur umständlich vermittelt werden können. Die intuitive Übermittlung wie etwa durch Körperreaktionen wie ein Lächeln oder lautes Lachen ist nicht möglich. Die Internet-Community hat sich Möglichkeiten geschaffen, diesem Umstand abzuhelfen: Emoticons, Akronyme und Verschriftlichung als Sound- und Aktionswörter. Die Darstellung nonverbaler emotionaler Kommunikationsinhalte kann durch bestimmte Zeichenkombinationen, sog. Emoticons (emotional icons) oder Smileys erfolgen. Ein Lächeln wird beispielsweise mit der Zeichenfolge : - ) dargestellt, die mittlerweile so weit verbreitet ist, dass die meisten Textverarbeitungs- oder Messaging-Anwendungen diese auomatisch umwandeln in $\odot$.

Lauthafte Reaktionen können wie in Comics in Form von Soundwörter („hmmm“, „argh“, „tss“ etc) wiedergegeben werden. Eine ähnliche Darstellungsform hat sich auch für mimische oder gestische Reaktionen durchgesetzt: diese werden durch Aktionswörter dargestellt, die sich vom Infinitiv des entsprechenden Verbs ableiten und in Sternchen ,eingeklammert' werden. Statt ,jetzt lächle ich“ wird also *lächel* geschrieben.

Darüber hinaus ist eine große Anzahl an $\underline{\text { Akronymen }}$ weit verbreitet, die sowohl der Darstellung bestimmter Sachverhalte als auch als verkürzte Schreibweise feststehender Redewendungen dienen können. Die Akronyme setzen sich entweder aus den Anfangsbuchstaben einer Wortfolge zusammen (z.B. „LOL“ für „laughing out loudly“; IMHO für „,in my humble opinion“) oder vom Wortlaut (CU für „,see you“, 4U anstelle von „for you“").

\subsubsection{Entzeitlichung und Enträumlichung}

Im Gegensatz zur herkömmlichen Kommunikation ist computervermittelte Kommunikation sowohl zeitlich als auch räumlich ungebunden. Personen, die via Internet miteinander in Verbindung treten müssen nicht am selben Ort, ja nicht einmal im selben Land oder auf demselben Kontinent sein, um gleichzeitig miteinander zu sprechen bzw. Textbotschaften auszutauschen. Wie auch das Telefon ermöglicht das Internet also den synchronen Informationsaustausch zwischen Personen, die sich an verschiedenen Orten befinden. Anders als das Telefon ermöglicht das Internet aber nicht nur die Übertragung auditiver Informationen, sondern auch den Austausch von Dateien, Textnachrichten, Bild- und Toninformation. Misoch (2006, S.59) spricht davon, dass „der Internetnutzer (...) sich 
demnach an zwei (oder mehreren) Orten zugleich (befindet): zum einen am realgeografischen Ort, an welchem er vor dem Bildschirm an Computer sitzt, und zum anderen an den virtuellen "Orten" im Cyberspace, die sich "hinter" dem Bildschirm eröffnen und in denen er kommuniziert, spielt usw. So kommt es zu einer Vermischung bzw. Überlappung von realem und virtuellem Raum, und der Kommunizierende kann sowohl im realen als auch im virtuellen Raum zugleich kommunizieren und des Weiteren in mehreren virtuellen Räumen gleichzeitig kommunikativ aktiv sein“ (Misoch 2006, S.59). Diese Parallelexistenz mehrerer Kommunikationswelten kann dazu führen, dass die einzelnen Kommunikationsräume subjektiv an Bedeutung verlieren und austauschbar oder ersetzbar erscheinen.

Nicht nur synchrone, auch asynchrone, also zeit-ungebundene Kommunikation ist via Internet möglich. Per E-Mail beispielsweise können Botschaften verschickt werden, die der Empfänger zu jeder beliebigen Zeit lesen und beantworten kann. Ein weiterer Aspekt der Entzeitlichung ist die Tatsache, dass computervermittelte Kommunikation meist schriftlich erfolgt und die ausgetauschten Nachrichten zentral oder lokal gespeichert werden, so dass sie jederzeit wieder eingesehen werden können. So kann der Gesprächsverlauf auch Stunden oder gar Jahre nach dem eigentlichen Kommunikationszeitpunkt nachvollzogen werden.

\subsubsection{Entkontextualisierung}

Im Gegensatz zur Face-to-Face-Kommunikation ist computervermittelte Kommunikation relativ unabhängig vom Aufenthaltsort der Kommunizierenden. Wesentliches Merkmal computervermittelter Kommunikation ist gar die Tatsache, dass die Gesprächspartner sich nicht am gleichen Ort aufhalten, wodurch die Einbindung in einen lokalen und sozialen Kontext, wie sie bei der Face-to-Face-Kommunikation gegeben ist, verloren geht. Da der Kontext eine entscheidende Rolle bei der Kommunikation spielt (vgl. Ungeheuer, 1982), können hieraus Verständnisprobleme resultieren. Herrmann (2001, S.22) beschreibt dies folgendermaßen: "Das besondere Problem der Online-Kommunikation besteht darin, daß die unmittelbare Situation, in die die Kommunikationspartner jeweils eingebunden sind, weniger stark wahrnehmbar ist, als dies bei der Face-to-Face-Kommunikation der Fall ist. Der Mitteilende muß im Rahmen der Konzipierung einer Mitteilung ( .. ) nicht nur die beschränkten Ausdrucksmittel berücksichtigen, sondern genau einschätzen, welche Teile des Kontextes der Kommunikationspartner wahrnehmen bzw. wahrgenommen haben kann und welche Teile daher zu explizieren sind."

$\mathrm{Zu}$ der kontextuellen Loslösung der Kommunizierenden kommt ein weiteres Problem: die 
„Entkontextualisierung der Inhalte“ (Misoch, 2006, S. 60). Bei internetbasierter Kommunikation werden Informationen aus ihrem Kontext gerissen und in einen neuen Zusammenhang, ,in einen neue netzartige Struktur, in ein Gewebe von Inhalten und Bedeutungen, eingefügt“ (Misoch, 2006, S. 60). Dadurch wird die Einordnung der Informationen durch den Empfänger erschwert, was wiederum dazu führen kann, dass sich neue Zusammenhänge und Interpretationsmöglichkeiten ergeben, andererseits aber auch ein Gefühl der Orientierungslosigkeit entstehen kann. Medienkritiker wie etwa Postman (1992, 1999) gehen daher davon aus, dass computervermittelte Kommunikationsinhalte lediglich oberflächlich verarbeitet werden und sehen die Technologisierung als Anzeichen eines drohenden Untergangs des Abendlandes.

\subsubsection{Digitalisierung}

Kommunikation via Internet ist per definitionem digital, was bedeutet, dass die ausgetauschten Botschaften gespeichert und somit auch lange nach dem eigentlichen Informationsaustausch noch eingesehen werden. Außerdem können die so gespeicherten Informationen problemlos weiterverschickt werden. Informationen, die via Internet ausgetauscht werden, sind daher tendenziell weniger gesichert als auf anderem Wege ausgetauschte Informationen. Des Weiteren kann der Empfänger sich bei computervermittelter Kommunikation weniger sicher sein, dass die Information tatsächlich von dem Kommunikationspartner stammt und nicht von diesem aus einer anderen digitalen Quelle empfangen und lediglich weitergeleitet wurde.

Darüber hinaus besteht bei computervermitteltem Informationsaustausch die Möglichkeit, verschiedene Kommunikationsmodi miteinander $\mathrm{zu}$ kombinieren und also beispielsweise neben Textbotschaften auch Audio- oder Videoinformationen auszutauschen.

Ein weiterer Aspekt der Digitalisierung ist die damit einhergehende Schnelligkeit des Informationsaustauschs: Die Datenübertragung erfolgt unabhängig von der räumlichen Distanz zwischen Sender und Empfänger mit enormer Geschwindigkeit.

\subsection{Theorien computervermittelter Kommunikation}

Wie bereits oben beschrieben, unterscheidet sich computervermittelte Kommunikation in wesentlichen Merkmalen von Face-to-face-Kommunikation. Verschiedene Theorien und zahlreiche Untersuchungen beschäftigen sich mit der Frage wie es um den emotionalen 
Gehalt dieser Form der Kommunikation gestellt ist. Im Folgenden sollen drei Theorien vorgestellt werden, die sich bereits sehr früh mit dieser Frage befasst haben und die jeweiligen wesentlichen Forschungsergebnisse erläutert werden. Die dargelegten Theorien stammen aus dem Bereich betrieblicher Kommunikation, wo die Computer bereits eingesetzt wurden, lange bevor durch den Heim-Computer und das Internet auch private Kommunikation medialisiert wurde.

\subsubsection{Social Presence Theory}

Die Theorie der sozialen Präsenz wurde bereits vor mehr als 30 Jahren von Short und Kollegen (Short, 1974; Short, Williams \& Christie, 1976) vorgelegt und ist Grundlage zahlreicher späterer Theorien der Kommunikation via neue Medien. Als ,soziale Präsenz' bezeichnen die Autoren ein Gefühl der Zugehörigkeit und Interaktion zwischen Menschen im Rahmen einer interpersonellen Beziehung. Dieses Gefühl kann nicht nur durch tatsächliche Präsenz erreicht werden, sondern auch bei medienvermittelter Kommunikation, sofern das Medium ein Gefühl von Wärme vermitteln kann. Dies ist in umso größerem Maße möglich je mehr das Medium von den Feinheiten sozialer Interaktion übermitteln kann. Dieser Theorie zu Folge sollte also eine Videokonferenz ein höheres Maß an sozialer Präsenz erzeugen als ein Telefonat, am geringsten sollte die wahrgenommene soziale Präsenz bei textgebundener computervermittelter Kommunikation wie etwa dem Chatten oder dem Austausch von EMails sein.

Die Theorie der sozialen Präsenz ist bis heute Gegenstand zahlreicher Untersuchungen, wie etwa der Arbeit von Perse \& Courtright (1993). Die Autoren stützen sich bei ihrer Untersuchung der Theorie sozialer Präsenz auf die Annahme, dass Individuen die zur Verfügung stehen den Kommunikationsmöglichkeiten kritisch bewerten und dann denjenigen Modus wählen, der ihren Bedürfnissen am nächsten kommt. Die Untersuchungsteilnehmer wurden aufgefordert anhand einer fünfstufigen Likert-Skala $\mathrm{zu}$ bewerten, wie persönlich, warm und sozial sie die 12 dargestellten Kommunikationsmöglichkeiten finden und inwiefern die verschiedenen Möglichkeiten ihren Bedürfnissen entsprechen. Die Ergebnisse sprechen eindeutig für die Social Presence Theory: als am ehesten in der Lage die persönlichen Bedürfnisse zu erfüllen wurde Konversation im sozialen Kontext eingestuft, dicht gefolgt von telefonischer Kommunikation; den geringsten Wert auf dieser Dimension erzielte computervermittelt Kommunikation. Trotz der Stringenz der Ergebnisse müssen diese mit Vorsicht interpretiert werden: $\mathrm{Zu}$ dem Zeitpunkt der Untersuchung war computervermittelte Kommunikation noch relativ unbekannt, das Internet noch kaum genutzt. 
Eine neuere Untersuchung zu dieser Fragestellung (Papacharissi \& Rubin, 2000) kommt zu weniger eindeutigen Befunden. Erhoben wurden Motive, die die persönlichen Handlungen beeinflussen, die Position im Leben (und die subjektive Zufriedenheit damit) sowie soziale Ängstlichkeit und selbst wahrgenommene Wertschätzung innerhalb des sozialen Nahraums. Insgesamt wurde das Internet von allen Teilnehmern am häufigsten zur Informationsbeschaffung genutzt. Die Einschätzung sozialer Präsenz bei computervermittelter Kommunikation war abhängig von persönlichen Merkmalen und beeinflusste wiederum das Nutzungsverhalten, insofern dass solche Personen, die eher zu sozialer Ängstlichkeit neigen, das Internet mehr zum interpersonalen Kontakt nutzen, was wiederum mit erhöhter Internetnutzung einhergeht. Fraglich ist hier allerdings, ob wirklich die persönlichen Merkmale Erklärung für die exzessivere Nutzung sind oder die ausgedehnten Nutzungserfahrungen mit zur positiveren, wärmeren Wahrnehmung computervermittelter Kommunikation beitragen.

Wie die Untersuchungsergebnisse zeigen so ist die Theorie der Sozialen Präsenz zumindest in der Zeit ihrer Entstehung zutreffend. Mit zunehmender Verbreitung des Internets und damit auch der computervermittelten Kommunikation lässt sich das Gefühl der sozialen Präsenz allerdings nicht mehr allein durch Eigenschaften des Kommunikationsmediums erklären, sondern wird auch bedingt durch Persönlichkeitsmerkmale und persönliche Erfahrungen mit dem jeweiligen Medium.

\subsubsection{Media Richness Theory}

Die Media Richness-Theorie von Daft \& Lengel (1986) geht ähnlich wie die Social Presence Theory davon aus, dass sich Medien in dem Ausmaß übermittelter sozialer Reize unterscheiden. Im Gegensatz zur zuvor geschilderten Theorie ist die Reichhaltigkeit an Informationen aber nicht subjektiv - wie die soziale Präsenz - sondern bezieht sich auf die objektiven Merkmale der Kommunikation wie etwa die Anzahl der angesprochenen Sinne und die Möglichkeiten zur direkten Reaktion (vgl. Rice \& Shook, 1990). 


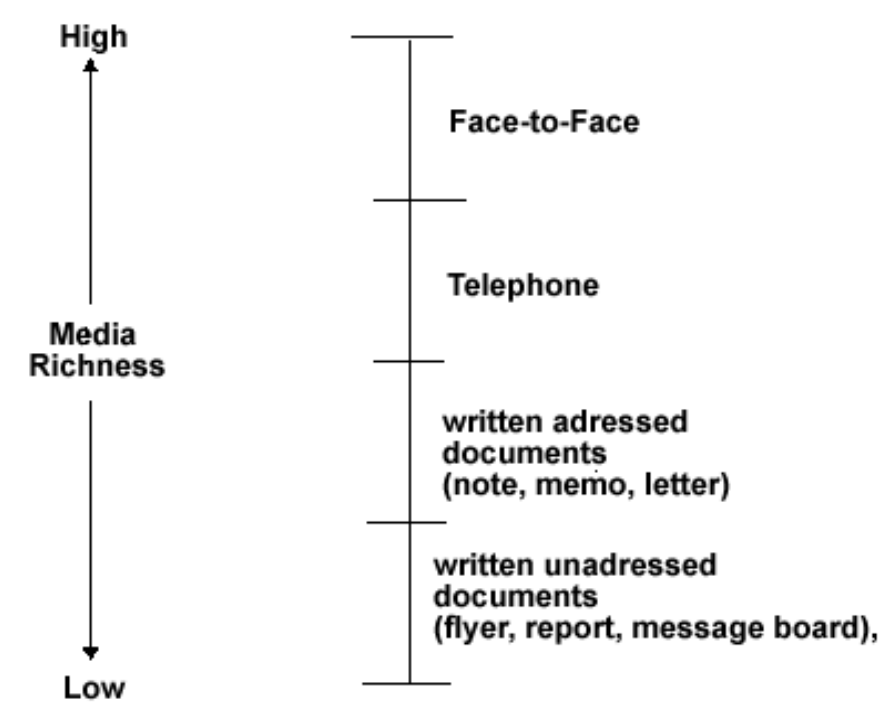

Abb. 5: Hierarchie der Media Richness (nach Daft, Lengel \& Trevino, 1987)

Eine Studie von Rice und Love aus dem Jahre 1987 untersucht den sozio-emotionalen Gehalt betrieblicher computervermittelter Kommunikation. Konkret handelt es sich um eine Inhaltsanalyse aller ausgetauschten Nachrichten in einer öffentlichen Computerkonferenz, wobei die Inhalte auf sozio-emotionalen Gehalt versus Aufgaben-Zentriertheit untersucht wurden. Die Ergebnisse zeigen einen deutlichen Anstieg des emotionalen Gehaltes mit steigender Anzahl ausgetauschter Botschaften - was wiederum darauf hindeutet, dass persönliche Erfahrungen die Wahrnehmung eines Mediums und das Verhalten in diesem Medium beeinflussen. Darüber hinaus lässt sich feststellen, dass der Grundton in emotional gefärbten Botschaften meist positiv war. Das Kodierschema, das zur Analyse benutzt wurde, war jedoch nicht ausgefeilt genug und erlaubte beispielsweise nur eine dichotome Kategorisierung in aufgaben-zentriert vs. emotional; da es nicht möglich war, eine Nachricht auf beiden Dimensionen positiv einzustufen, ist davon auszugehen, dass der Anteil der als emotional klassifizierten Nachrichten unterschätzt wurde.

\subsubsection{Lack of Social Context and Cues Theory}

Eine weitere Theorie stammt von Kiesler und Kollegen aus dem Jahre 1984 und beschäftigt sich mit den sozialpsychologischen Aspekten der computervermittelten Kommunikation. Die Autoren postulieren, dass im Kontext computervermittelter Kommunikation soziale Hinweisreize und Kontextinformationen nur begrenzt vorliegen und dass keine konkreten Verhaltensregeln und -normen existieren. Die Kommunikation sei folglich depersonalisiert 
und durch Anonymität, herabgesetzte Self-awareness und verringerte Selbstregulation gekennzeichnet und schaffe ein Umfeld, dass die Nutzer dazu verleite „Amok zu laufen“. Postuliert wird also, dass computervermittelte Kommunikation eher zielorientiert als emotional gefärbt ist, dass Entfremdung und Feindseligkeit vorherrschen. Eine von den Autoren (Kiesler et al, 1984) durchgeführte Untersuchung sollte diese Hypothese testen, wobei Gruppen von jeweils drei Personen zu einer Übereinstimmung bei einer bestimmten Fragestellung kommen sollte. Die Kommunikation zur Kompromissfindung fand entweder per Computer oder im Sechs-Augen-Gespräch statt. Tatsächlich brauchten Personen, die nicht persönlich, sondern via Computer kommunizierten, nicht nur länger um zu einer Entscheidung zu kommen, sondern benutzten auch häufiger Flüche, Beschimpfungen und feindselige Kommentare. Diese Ergebnisse müssen allerdings mit Vorsicht betrachtet werden, da Kiesler und Kollegen weder das experimentelle Vorgehen noch die Auswertung genau beschreiben.

Eine neuere Untersuchung (Kraut, Patterson, Lundmark, Kiesler, Mukopadhyay \& Scherlis, 1998) geht von ähnlichen Annahmen aus wie Kiesler und Kollegen und beschäftigt sich mit der Frage, ob die Internetnutzung soziale Beziehungen positiv oder negativ beeinflusst. Zur Beantwortung dieser Fragestellung wurden 169 Teilnehmer aus 73 verschiedenen Haushalten über einen Zeitraum von einem bzw. zwei Jahren beobachtet. Die Teilnehmer hatten zuvor noch keine Erfahrung mit dem Internet gemacht und wurden zu Beginn der Studie in den Umgang mit dem neuen Medium eingewiesen und mit der nötigen Hard- und Software ausgestattet. Demographische Informationen, der Grad sozialer Eingebundenheit und das psychische Wohlergehen wurden sowohl zu Beginn der Untersuchung als auch nach 12 und 24 Monaten erfasst. Darüber hinaus wurde die Internetnutzung der einzelnen Teilnehmer im Untersuchungszeitraum erhoben.

Die Auswertung der Daten zeigte, dass die Internetnutzung bei einer Konstanthaltung der Ausgangswerte Auswirkungen auf die soziale Einbindung und das psychische Wohlergehen hatte. Auch die Kommunikation innerhalb der Familie wird durch die Internetnutzung beeinflusst, was die Autoren wie folgt angeben: „for our purposes, the most important finding is that greater use of the Internet was associated with subsequent declines in family communication“ (Kraut et al., 1998, S. 1025). Aber nicht nur innerhalb der Familie, auch im weiteren sozialen Umfeld nimmt das Ausmaß der Einbindung und Kommunikation mit zunehmender Internetnutzung ab.

Zum psychosozialen Wohlergehen der Nutzer lässt sich sagen, dass (bei statistischer Berücksichtigung der Werte zum Erhebungsbeginn) häufige Internetnutzung mit größerer 
selbst berichteter Einsamkeit einhergeht. Darüber hinaus berichten solche Personen von einer signifikant höheren Anzahl an Stressoren im Alltag als Personen mit geringerer Internetnutzung, wobei sich objektiv keine Zunahme der Stressoren belegen ließ. Es kann also von einer höheren Empfänglichkeit der Personen gegenüber Stress ausgegangen werden. Schließlich ließ sich auch ein Zusammenhang zwischen ausgedehnter Internetnutzung und Depressivität nachweisen, wobei allerdings auch andere Faktoren wie das Ausmaß des Alltagsstresses zu Beginn der Untersuchung, eine Rolle spielten.

\subsection{Zusammenfassende Bewertung}

Die drei dargestellten Theorien und die dazugehörigen Untersuchungsergebnisse legen die Vermutung nahe, dass computervermittelte Kommunikation auf andere Art und Weise wahrgenommen und verarbeitet wird als Kommunikation im persönlichen Kontakt. Es fehlt an sozialer Wärme und dieser Mangel an Nähe, Personalität und klaren Verhaltensregeln scheint $\mathrm{zu}$ unsozialem, aggressivem Verhalten zu führen, wofür auch die Bandbreite und Verbreitung von Cyber-Kriminalität sprechen (vgl. Bremer, 2000; Schetsche, 2001). Darüber hinaus wirkt sich die Internetnutzung auch negativ auf soziale Kontakte im realen Leben aus. Zwar sind die beschriebenen Studien größtenteils aus einer Zeit, in der das Internet und damit auch computervermittelte Kommunikation in unserer Gesellschaft noch nicht so weit verbreitet waren, wie dies heute der Fall ist. Auch wenn etwas Gefühl der sozialen Kälte computervermittelter Kommunikation mit der Nutzungsdauer und unter dem Eindruck individueller Erfahrungen abnimmt (vgl. King \& Xia, 1999), so kann doch davon ausgegangen werden, dass die beschriebenen Phänomene - wenn auch in abgeschwächter Form - weiter bestehen. Dies wirft die Frage auf, inwiefern der Einsatz computervermittelter Kommunikation im Rahmen klinisch-therapeutischer Intervention angebracht ist oder ob nicht vielmehr Internetnutzung und der Gebrauch computervermittelter Kommunikation erst zu einem Zustand führt, der therapeutische Intervention nötig macht. Diesen Fragen soll im Folgenden nachgegangen werden. 


\section{Die Rolle des Internets in der klinischen Psychologie und Psychotherapie}

Mit zunehmender Verbreitung des Internets wurde auch dessen Bedeutung für die Wissenschaft und Forschung immer größer. Im Folgenden soll dargestellt werden, welche Rolle das Internet für die Wissenschaften allgemein und für die Psychologie im Besonderen hat. Der Fokus soll dabei auf den Schnittstellen zwischen dem Internet und dem Teilbereich der Klinischen Psychologie/Psychotherapie liegen.

Das Internet ist aus dem wissenschaftlichen Betrieb nicht mehr wegzudenken, dies gilt auch aber nicht ausschließlich - für die Medizin und die Psychologie. Hahn \& Günter (1999) haben verschiedene Funktionen des Internets identifiziert:

1. Zum einen ist das Internet Informationsquelle für Wissenschaftler und Laien, es unterstützt die wissenschaftliche Kommunikation über Ländergrenzen hinweg, ist

2. Medium für Publikationen und bietet Möglichkeiten zur Literatursuche.

3. Darüber hinaus dient es als Präsentationsplattform für Fachgesellschaften, Verbände und Institute, sowie Kliniken.

Auch wenn Hahn \& Günter bei ihrer Bestandsaufnahme die Funktionen des Internets aus dem Blickwinkel der Psychologie betrachten, so kann doch festgehalten werden, dass dies globale Funktionen sind, von denen auch andere Wissenschaften Gebrauch machen. Für therapeutische Forschung insbesondere ist das Internet aber auch aus anderen Gründen von Interesse: nicht nur als Medium für die Betreibung der Wissenschaft, sondern auch als deren Gegenstand. Fragen des Wissensmanagements und der Wissensverbreitung im digitalen Zeitalter, Besonderheiten der computervermittelten Kommunikation, Anonymität und Pseudonymität, Gruppenbildungsprozesse im Cyberspace sowie differentiell Nutzungsaspekte sind schon lange im Fokus der Forschung.

Neben diesen Aspekten, mit denen sich unterschiedliche Fachbereiche der Psychologie beschäftigen, dient das Internet in der Klinischen Psychologie und Psychotherapie auch als therapeutisches Medium. 


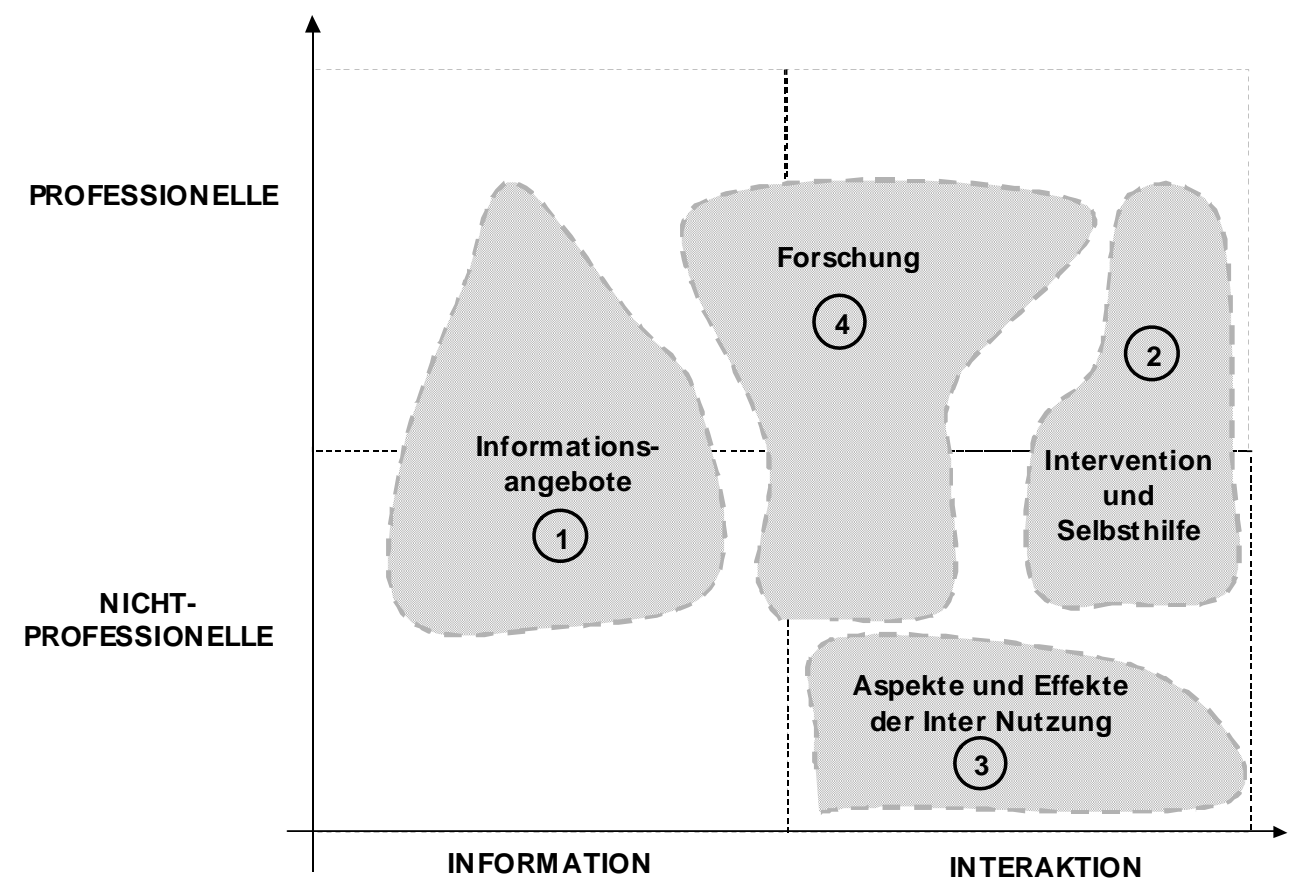

Abb. 6: Schnittstellen der Klinischen Psychologie mit dem Medium Internet; die Zahlenangaben der Felder stehen für 1 = Informationsangebote; 2 = Intervention und Selbsthilfe, 3 = Aspekte und Effekte der Internetnutzung 4 = Forschung (modifiziert nach Ott, R. \& Eichenberg, C., 2003, S. 14)

Ott \& Eichenberg (2003) haben vier Schnittstellen zwischen dem Internet und dem Teilbereich der klinischen Psychologie identifiziert: 1. Informationsangebote, 2. Intervention und Selbsthilfe, 3. Aspekte und Effekte der Internetnutzung und 4. Forschung (vgl. Abb. 6). Diese vier Überlappungsbereiche ordnen die Autoren in ein Vierfelder-Schema, in dem sie zwischen professionellen und Laien-Akteuren sowie und dem Ziel der Angebote Information vs. Interaktion - unterscheiden.

$\mathrm{Zu}$ 1. Als Informationsmedium bietet das Internet eine große Anzahl an Informationen zu psychischen Störungen und Interventionen sowohl für Laien als auch für Professionelle. In einem eigenen Projekt (Kap. 4.3) soll erläutert werden, wie dieses Informationsangebot von Personen mit psychischen Störungen und deren Angehörigen genutzt werden kann. Ein Vorteil dieser virtuellen Informationsangebote ist die leichte Zugänglichkeit für jeden Interessierten. Ein Nachteil ist darin zu sehen, dass es keine Qualitätssicherung im Internet gibt und somit unter Umständen die Gefahr besteht, falsche Informationen zu erhalten. Hier besteht somit eine hohe Verantwortung des Autors. 
$\mathrm{Zu}$ 2. Das Internet ist aber nicht nur Informations-, sondern auch Kommunikationsmedium und kann als solches nicht nur zur Wissensvermittlung genutzt, sondern auch zu Zwecken der Selbsthilfe und psychologischen Intervention genutzt werden. Selbsthilfe erfolgt meist im Erfahrungsaustausch mit anderen Betroffenen, in der überwiegenden Zahl der Fälle per Webforum oder Newsgroup, selten auch per Chat.

Das Angebot an therapeutischer Intervention muss weiter differenziert werden: zu unterscheiden sind hier Beratungsangebote und internetbasierte Therapieformen. Im Bereich der Beratung ist wiederum eine Differenzierung notwendig zwischen kommerziellen und nicht-kommerziellen Angeboten, wobei letztere meist von gemeinnützigen Einrichtungen wie etwa profamilia, der Arbeiterwohlfahrt oder der Telefonseelsorge angeboten werden. Beratungsangebote sind in den meisten Fällen unspezifischer Natur, d. h. sie bieten Personen mit vielfältigen Problemen Rat. Die Kommunikation erfolgt per E-Mail oder Chat.

Internetbasierte Therapieangebote dagegen sind störungsspezifisch. Auch hier erfolgt der Austausch zwischen Patient und Therapeut per E-Mail oder Chat, in seltenen Fällen sind auch Videokonferenzen oder eine Kommunikation per SMS vorgesehen.

$\mathrm{Zu}$ 3. Als dritte Schnittstelle zwischen klinischer Psychologie und Internet haben Ott \& Eichenberg (2003) die klinisch-psychologischen Aspekte der Internetnutzung identifiziert: Themenfelder in diesem Bereich sind pathologischer Internetgebrauch, Einfluss der Anonymität bzw. Pseudonymität der Internet-Kommunikation auf Verhalten und Selbstwahrnehmung, insbesondere im Zusammenhang mit der Multiple Personality Disorder.

$\mathrm{Zu}$ 4. $\mathrm{Zu}$ guter letzt wird das Internet auch verstärkt in der psychologischen Forschung eingesetzt. Damit ist zum einen der Austausch zwischen Forschern via Internet gemeint, wozu das Internet ursprünglich entwickelt wurde. Zum anderen werden aber auch Forschungsergebnisse im Internet publiziert und sogar ganze Untersuchung über das Internet durchgeführt, da sich hier ein großer Pool an Probanden offenbart und die Durchführung computergestützt äußerst ökonomisch ist, wenngleich die Validität sicherlich häufig fragwürdig ist.

All dies sind zweifelsohne äußerst spannende Themen, die in den letzten Jahren in der Psychologie immer mehr an Bedeutung gewonnen haben. Der Fokus der vorliegenden Arbeit soll jedoch auf der Betrachtung psychologischer Interventionsmöglichkeiten via Internet liegen. Dazu wird im Folgenden zuerst die Nutzbarkeit computervermittelter Kommunikation kritisch betrachtet. 


\section{Internetbasierte klinisch-psychologische Intervention}

Im Folgenden soll - nach einem kurzen Blick auf die historische Entwicklung und dem aktuellen Forschungsstand zu internetbasierter klinisch-psychologischer Intervention - auf die Qualitätsanforderungen an solche Konzepte eingegangen werden. Im Anschluss daran werden die unterschiedlichen Formen der internetbasierten psychologischen Hilfe, von Selbsthilfe über Beratung hin zur Online-Therapie, vorgestellt. Der Schwerpunkt wird dabei auf der Beschreibung etablierter Formen der Internet-Hilfe liegen.

\subsection{Entwicklung und Status Quo}

Die Angebote an computergestützten therapeutischen Interventionsmöglichkeiten sind zahlreich und breit gefächert. Unterschiede bestehen sowohl hinsichtlich des genutzten Internetdienstes, der angebotenen Interventionsform, der methodischen Ausrichtung des Angebotes, der Kosten und der Professionalität der Anbieter.

Bezüglich des ersten Unterscheidungskriteriums, des genutzten Internetdienstes, lässt sich sagen, dass Beratung und Therapie hauptsächlich per E-Mail-Kontakt, selten aber auch in Chatrooms oder IRC-Channels stattfinden. Im Gegensatz dazu werden im Bereich der Selbsthilfe und des Erfahrungsaustauschs mit anderen Betroffenen und Angehörigen insbesondere Internetforen und Chats genutzt.

Die Nutzung des Internets für therapeutische Intervention hat ihren Ursprung in den USA, wo im Jahre 1995 erste „question \& answer services“ entstanden (vgl. Grohol, 1998). In den folgenden Jahren ist die Zahl der Anbieter drastisch gestiegen und auch das Angebot wurde verbreitert. Dies ist damit zu erklären, dass nicht nur die Nachfrage nach Online-Beratung und -Therapie sehr hoch ist (Grohol, 1998, S. 128), sondern diese Angebotsart auch für den Anbieter Vorteile bereithält. Da per Internet erwartungsgemäß mehr Personen angesprochen werden können und die laufenden Kosten niedriger sind als bei einer Niederlassung in einer herkömmlichen Praxis, verspricht das Online-Geschäft eine deutlich größere Gewinnspanne. Dies und die Tatsache, dass Hilfesuchende sich meist nur unzureichend informieren bzw. sich bei ihren Nachforschungen auf die Informationen verlassen, die der Anbieter selbst auf seiner Homepage bereitstellt, führt dazu, dass insbesondere im Bereich kommerzieller Angebote die Anzahl an Anbietern mit unzureichender Qualifizierung hoch ist. Döring (2000, S. 535) spricht daher von einem „,besonders schwer durchschaubaren Psycho-Markt mit spezifischen ethischen Probleme“. 
Zusätzlich erschwert wird die Situation dadurch, dass es keine verbindlichen Richtlinien gibt, anhand derer die Qualität von Internet-Angeboten zur Beratung oder Therapie bewertet werden können. Zwar sind zwischenzeitlich einige wissenschaftliche Arbeiten erschienen, die sich mit diesem Thema befassen (Hinsch \& Schneider, 2002; Knatz, 2006; van Well, 2000), diese sind aber den meisten Hilfesuchenden nicht bekannt. So ist bei der Auswahl und Inanspruchnahme kommerzieller Hilfsangebote stets Vorsicht geboten.

Im Bereich der kostenlosen Angebote ist die Zahl unseriöser Anbieter verständlicherweise wesentlich geringer (vgl. Janssen, 1998). Diese nicht-kommerziellen Angebote stammen meist von gemeinnützigen Einrichtungen wie pro familia (http://www.profamilia.de) oder der Telefonseelsorge (http://www.telefonseelsorge.de). Auch Erziehungsberatungsstellen bieten teilweise zusätzlich zu ihrem Angebot vor Ort auch virtuelle Beratung an (z. B. http://www.helpmails.de).

Unter anderem auch aufgrund der geschilderten Problematik ist die Zukunft der OnlineBeratung und -Therapie bislang noch ungewiss. Hinzu kommt, dass bislang zur Diagnostik und Verlaufskontrolle herkömmliche standardisierte Fragebögen eingesetzt werden, deren Validität und Reliabilität im speziellen Kontext der Internetberatung und -therapie noch nicht ausreichend überprüft wurde.

„Wird es eines Tages eine Form der Netztherapie mit spezifischem Setting sowie genuin neuen computerbasierten Diagnose- und Interventionsmethoden geben? Oder werden die einzelnen Therapierichtungen und unterschiedlicher Weise die Optionen des Netzes integrieren“?

(Döring, 2000, S. 537).

Lange Zeit war die Zukunft der internetbasierten psychologischen Intervention ungewiss. In der Euphorie der Anfangszeit wurden hauptsächlich solche Konzepte entwickelt, bei denen die Intervention vollständig über das Internet abgewickelt wurde - vom Erstkontakt bis zur Abschlusssitzung. Nicht immer konnten diese Konzepte Erfolge verbuchen. Dies ist zum einen darauf zurückzuführen, dass nicht alle Störungen gleich gut auf eine Intervention ohne persönlichen Therapeutenkontakt ansprechen (vgl. Ott, 2003). Zum anderen waren diese Konzepte noch nicht so ausgereift wie neuere Interventionsformen. Hinzu kam oft, dass gerade in dieser frühen Phase der Entwicklung solche Projekte noch weitgehend unbekannt waren und daher in der Zielgruppe, den potentiellen Patienten, vor allen Dingen aber unter niedergelassenen oder Kliniktherapeuten auf Skepsis gestoßen sind. 
Die Entwicklungen in den letzten Jahren waren in dieser Hinsicht durchaus positiv: Durch eine steigende Zahl an Wirksamkeitsstudien konnten bestehende Konzepte optimiert werden und positive Befunde konnten das Ansehen solcher Interventionsformen deutlich verbessern. Darüber hinaus wurden neue Ansätze erdacht, die nicht nur allein auf computervermittelte Kommunikation setzten, sondern diese mit herkömmlicher Behandlung kombinieren. Gerade für die Nachsorge nach einer stationären Behandlung scheint sich eine „Internetbrücke“ (Kordy, Golkaramnay, Wolf, Haug \& Bauer, 2006) gut zu eigenen, da der Kontakt zwischen Patient und Therapeut auf diese Weise nicht abrupt enden muss, sondern reduziert und in den Alltag des Patienten integriert werden kann.

Eine weitere neue Entwicklung ist der Einsatz von Textnachrichten (SMS) ebenfalls in der Nachbehandlung (z.B. Bauer, Percevic, Okon, Meerman \& Kordy, 2003; Robinson, Perkins, Bauer, Hammond, Treasure \& Schmidt, 2006). Die Befunde hierzu sind jedoch nicht eindeutig (vgl. Kap. 5.2.4) und der Ansatz scheint weiterer Forschung und Verbesserung zu bedürfen.

\subsection{Qualitätsanforderungen an Online-Angebote zur therapeutischen Intervention}

Die oben geschilderte Problematik der unzureichenden und schwer zu überprüfenden Qualifizierung der Anbieter kommerzieller Online-Beratungsangebote hat dazu geführt, dass zahlreiche wissenschaftliche Publikationen zu diesem Thema erstellt worden sind. Unter anderem beschäftigt sich eine gesamte Ausgabe des e-beratungsjournal.net (2006, 2(1)) mit diesem Thema. Dies hat ein Nebeneinander unterschiedlicher Kriterienkataloge zur Folge (wie den von Knatz, 2006; s.u.), die jedoch wirkungslos bleiben, solange es keine zentrale Einrichtung gibt, die deren Umsetzung einfordert und überprüft. Bis dahin müssen Ratsuchende bei der Auswahl von internetbasierten Beratungs- und Therapiestellen auch weiterhin äußerst sorgfältig vorgehen. Hilfestellung dabei kann beispielsweise das Gütesiegel geben, das der Bundesverband deutscher Psychologinnen und Psychologen (BDP) für OnlineBeratung entwickelt hat.

Im Folgenden sollen sowohl der von Knatz entwickelte Kriterienkatalog (beispielhaft für zahlreiche andere Abhandlungen $\mathrm{zu}$ diesem Thema) als auch das Gütezeichen des Bundesverbands deutscher Psychologinnen und Psychologen vorgestellt werden. 
Das Qualitätszeichen des BDP soll “eine Orientierung zum Auffinden qualitativ hochwertiger Beratungen (...) geben“ (Homepage des BDP). Es wird auf den Internetseiten zertifizierter Anbieter angezeigt und ist mit einer Seite des BDP verlinkt, um die Fälschungssicherheit zu erhöhen. Die Vergabe des Gütesiegels ist mit der Erfüllung bestimmter Qualitätskriterien verbunden (vgl. Internetseite des BDP):

\section{$\S$ Kurzfristige Beantwortung von Anfragen}

E-Mail-Anfragen müssen innerhalb einer Frist von maximal 3 Tagen beantwortet werden. Diese Frist beginnt bei kostenlosen Angeboten mit dem Eingang der Anfrage, bei bezahlten Angeboten mit dem Zahlungseingang.

\section{$\S$ Hohe Qualifikation}

Das Gütesiegel wird nur an solche Berater verliehen, die einen Abschluss als DiplomPsychologe vorweisen können. Diese Ausbildung kann als Grundvoraussetzung für qualitativ hochwertige Beratung angesehen werden. Darüber hinaus muss eine Ausbildung in Gesprächsführung und Krisenintervention nachgewiesen werden.

\section{$\S$ Schweigepflicht, Anonymität und Vertraulichkeit}

Da das Qualitätszeichen nur an Diplom-Psychologen verliehen wird, sind die Berater sowohl durch das Strafgesetzbuch als auch durch die berufsethischen Verpflichtungen an die Schweigepflicht gebunden. Dies unterscheidet den Beruf des Psychologen von andern sozialen Berufen. Damit diese Pflichten auch in der Online-Beratung erfüllt werden können, sind technische Sicherheitsvorkehrungen notwendig. Träger des Gütezeichens müssen diese vorweisen können.

\section{$\S$ Verantwortlicher Umgang mit den Grenzen}

Die zertifizierten Anbieter beachten die Grenzen der Online-Beratung und weisen z. B. auf Ihrer Website darauf hin, dass diese nicht in allen Fällen eine adäquate Unterstützung bieten kann, da manche Probleme, insbesondere stark belastende psychische Krisen, persönlichen und längerfristigen Kontakt erfordern.

\section{$\S$ Technische Sicherheit}

Zur Gewährleistung des Datenschutzes und der Anonymität sind technische Grundkenntnisse erforderlich. Vor der Vergabe des Gütezeichens werden Sicherheitsstrategien bei der Datenübertragung und -speicherung geprüft. Dazu gehört auch, dass Hilfesuchende auf Sicherheitslücken hingewiesen werden, z. B. Verschlüsselungstechniken für E-Mails und die Verwendung von E-Mail-Adressen, die keine Rückschlüsse auf die Identität des Nutzers zulassen. 
Die Vergabe des Gütezeichens ist also an die Erfüllung bestimmter Mindestvoraussetzungen für sichere und kompetente Beratung geknüpft. Fraglich ist allerdings, ob dieses Qualitätssiegel den Hilfesuchenden ausreichend bekannt ist. Darüber hinaus muss festgehalten werden, dass die Zahl zertifizierter Anbieter bislang so gering ist (vgl. Liste zertifizierter Anbieter im Anhang), dass der Bedarf an Online-Beratung nicht von diesen Einrichtungen gedeckt werden kann.

Neben dem Bundesverband Deutscher Psychologen haben sich auch zahlreiche wissenschaftliche Arbeiten mit dem Thema auseinander gesetzt und Kriterienkataloge erstellt, die denen des BDP allerdings sehr ähnlich sind. Knatz (2006, S. 2) nennt folgende Punkte als Kriterien einer ,guten’ Beratungseinrichtung:

$\S \quad$ Transparenz der Institutionen nach innen und außen

$\S$ Kompetenz der BeraterInnen

$\S$ Zufriedenheit der Ratsuchenden

$\S$ Nutzung persönlicher und wirtschaftlicher Ressourcen

$\S$ Technische Fragen und Sicherheitsstandards.

Wie bereits erwähnt ist der praktische Nutzen solcher Kriterienkataloge für den Hilfesuchenden eher gering einzuschätzen.

Positiv zu bewerten ist dagegen die Initiative der Deutschen Hauptstelle für Suchtfragen (DHS), die eine „Checkliste für Nutzerinnen und Nutzer von Suchthilfeangeboten im Internet“ (s. Anhang) veröffentlicht hat. Da diese Einrichtung bei den Hilfesuchenden bekannter sein dürfte als psychologische Fachzeitschriften und der BDP, kann davon ausgegangen werden, dass auch diese Checkliste mehr Leser findet. Darüber hinaus scheint sie als Anleitung zur Auswahl einer Beratungseinrichtung wesentlich besser geeignet.

\subsection{Selbsthilfe}

Das Internet bietet psychisch Kranken und deren Angehörigen eine Vielzahl an Möglichkeiten: neben den professionellen Angeboten wie Beratung und Therapie gehört dazu ein breites Spektrum an Informations- und Selbsthilfeangeboten.

Informationsangebote, sowohl von Professionellen als auch von Laien und selbst Betroffenen, helfen den Erkrankten, sich Wissen über die Störung, unter der sie leiden, anzueignen. Dies kann eine hilfreiche Ergänzung zur Psychoedukation durch Fachkräfte sein, da hier das sprachliche Niveau sowie die Menge der Information selbst ausgewählt werden und zu jeder 
beliebigen Zeit - allein oder im Beisein nahe stehender Personen - abgerufen werden kann. Darüber hinaus können Erfahrungsberichte von anderen Betroffenen Mut machen und zu der Erkenntnis beitragen, dass man mit seinem Schicksal nicht allein ist.

Ein ähnliches Ziel verfolgen auch Online-Selbsthilfegruppen. Die Kommunikation erfolgt in den meisten Fällen solcher virtueller Selbsthilfegruppen über Internetforen, Mailinglisten oder Newsgroups, in selteneren Fällen auch über Chat. Diese virtuellen Selbsthilfegruppen können Personen die Kontaktaufnahme mit Gleichgestellten erleichtern, da sie niedrigschwelliger sind als herkömmliche Selbsthilfegruppen. Weitere Vorteile liegen darin, dass diese OnlineGruppen jederzeit und von jedem Ort aus erreichbar sind.

Zwar gibt es bislang kaum Studien, die sich mit der Wirksamkeit solcher OnlineSelbsthilfegruppen befassen und diese mit herkömmlichen Einrichtungen der Selbsthilfe vergleichen, die wenigen bislang existierenden Arbeiten zu diesem Thema sprechen aber dafür, dass die Funktionen von Selbsthilfe im virtuellen Raum ebenso gut erfüllt werden können wie im realen (vgl. King \& Moreggi, 1998).

Allerdings ist auch hier Vorsicht geboten. Zum einen ist von einer allzu großen Erwartungshaltung abzuraten, da Selbsthilfegruppen allein - sowohl im Internet als auch herkömmlicher Art - bei schweren Störungen und großem Leidensdruck keine Verbesserung herbei führen können (s. Döring, 1998), sondern allenfalls eine professionelle Intervention begleiten und unterstützen können. Zum anderen können auch die Nutzer oder der transportierte Inhalt zum Problem werden. Dies war in der Vergangenheit bei den sog. Magersuchtforen der Fall, in denen von den Teilnehmern falsche Wertvorstellungen vermittelt wurden, die den Leidensdruck der Betroffenen nicht verringert sondern eher vergrößert werden. Diese Gefahr besteht insbesondere bei Internetforen, die offen zugänglich sind; bei Mailinglisten und Newsgroups ist das Risiko geringer, da hier eine Anmeldung erforderlich ist und bei schweren Verstößen gegen die Gruppenregeln der Ausschluss aus der Gruppe erfolgen kann.

\subsection{Online-Beratung}

Nicht nur Selbsthilfe, auch Beratung ist via Internet möglich. Unter dem Begriff „Beratung“ werden hierbei eine Vielzahl von Kommunikationsmöglichkeiten verstanden. Es gibt, im Gegensatz zur Psychotherapie, keine allgemein gültige Standarddefinition des Begriffes im therapeutischen Sinne. Exemplarisch soll zum besseren Verständnis somit eine Definition für 
„psychologische Online-Beratung“, erstellt durch die FSP Kommission „Fortbildung OnlineBeratung) (Andermatt 2003), aufgezeigt werden.

„Psychologische Online Beratung ist eine aktive, helfende Begegnung resp. Beziehung zwischen einem/einer Ratsuchenden und einer/einem psychologischen BeraterIn. Sie findet virtuell im Internet mittels dessen spezifischen Kommunikationsformen (E-Mail, Chat, Forum etc.) statt, wobei die KlientInnen Ort und Zeitpunkt der Problemformulierung selbst bestimmen.

Sie hat zum Ziel, bei den KlientInnen kognitiv-emotionale Lernprozesse anzuregen, damit die Selbststeuerungs- und Handlungsfähigkeit wieder erlangt oder verbessert werden kann. Psychologische Online-BeraterInnen stützen Ihre Beratung auf anerkannte psychologischberaterische Methoden und halten sich an medienspezifisch erweiterte berufsethische Standards (Schweigepflicht, Datenschutz, Erkennbarkeit der Beraterkompetenz). “

Diese Beratung ist nach Sommers (1996; zitiert nach Döring, 2000) insbesondere für folgende Personen von Interesse:

$\S$ Personen, die nicht über die finanziellen Mittel verfügen, um sich eine herkömmliche Beratung leisten zu können

$\S$ Personen, die eine gewisse Distanz zu ihrem Berater halten wollen

$\S$ Personen, die in einer Region mit schlechter psychosozialer Infrastruktur leben und daher keine herkömmliche Beratungsstelle aufsuchen können

$\S$ Personen, die aufgrund körperlicher Einschränkungen herkömmliche Beratung nicht oder nur unter erschwerten Bedingungen in Anspruch nehmen können (z.B. Taubstumme, Schwerhörige, ans Haus Gebundene)

$\S$ Personen, die vor einer herkömmlichen Beratung zuerst unverbindlich Kontakt aufnehmen wollen

Eichenberg (2003) weist darauf hin, dass die von Sommers dargestellte Zielgruppenbeschreibung nicht weit genug gefasst ist und Online-Beratung auch den Bedürfnissen folgender Personen entgegenkommt:

$\S$ Personen, die Schwellenängste haben, eine herkömmliche Beratungsstelle aufzusuchen

$\S$ Personen, die aufgrund psychischer Einschränkungen (zeitweise) nicht in der Lage sind eine herkömmliche Beratungsstelle aufzusuchen (z. B. wegen Agoraphobie oder in einer akuten depressiven Phase)

$\S$ Personen, die sich bevorzugt schriftlich ausdrücken. 
Welche Möglichkeiten zur Online-Beratung solchen Menschen zur Verfügung stehen, soll im Folgenden erläutert werden.

\subsubsection{Angebot psychologischer Online-Beratung}

Einen ersten Hinweis auf die Verbreitung von Online-Beratungsangeboten kann die Abfrage in Internet-Suchmaschinen geben, auch wenn die so gewonnenen Zahlen nicht zuverlässig sind und das Angebot hier meist systematisch unterschätzt wird (vgl. Günther \& Hahn, 2000). Tabelle 2 zeigt einen Überblick über die erzielten Treffer in drei bekannten Suchmaschinen. Die Zahlen scheinen erstaunlich hoch, vor allem bei einem Vergleich mit der von Eichenberg (2003) durchgeführten Suchanfrage, wo die Zahl der erzielten Treffer noch nicht einmal ein Zehntel der hier dargestellten ausmacht. Das spricht für eine deutliche Zunahme des Angebots in den letzten Jahren.

Tab. 2: Treffer zu den Begriffen „Online-Beratung“ und „Psychologische Online-Beratung“ in den Internet-Suchmaschinen Google, Fireball und Yahoo (20.05.2007)

\begin{tabular}{lll}
\hline Suchmaschine & Online-Beratung & Psychologische Online-Beratung \\
\hline www.google.de & 1.190 .000 & 90.100 \\
www.fireball.de & 3.287 .783 & 35.164 \\
www.yahoo.com & 33.700 .000 & 582.000 \\
\hline
\end{tabular}

Neben der großen Zahl an Angeboten ist auch deren Bandbreite zu beachten: zu unterscheiden sind nicht nur allgemeine von speziellen (z.B. Paarberatung, Berufsberatung) Angeboten, auch die Qualität der Beratung und die Kosten differieren stark zwischen den Anbietern. Wie bereits oben beschrieben, sind vor allem im Bereich kommerzieller Angebote große Unterschiede bezüglich der fachlichen Ausbildung der Berater und der Qualität der Beratung $\mathrm{zu}$ bemerken, so dass hier besondere Vorsicht bei der Auswahl geboten ist. Im Anhang befindet sich eine Auswahl von deutschsprachigen Online-Beratungsangeboten, die die Bandbreite der Professionalität und Kosten veranschaulicht. 
Da hier aufgrund der oben dargestellten Bandbreite von Online-Beratungsangeboten kein repräsentatives Bild gegeben werden kann, soll stattdessen im Folgenden eine OnlineBeratungseinrichtung aus dem Bereich der nicht-kommerziellen, gemeinnützigen Angebote vorgestellt werden, die sich durch ihre Qualität und wissenschaftliche Überprüfung auszeichnet und auf eine mehrjährige erfolgreiche Bilanz zurückblicken kann.

\subsubsection{E-Mail-Beratung der Katholischen Telefonseelsorge}

Wie bereits oben erwähnt ist ein Großteil der kommerziellen Angebote an therapeutischer Online-Beratung von unzureichender oder nicht überprüfbarer Qualität. Nur wenige OnlineBerater sind mit dem Gütesiegel des Berufsverbandes Deutscher Psychologen ausgezeichnet. Ein Lichtblick im Dschungel der Online-Beratungsstellen sind daher die kostenlosen Angebote gemeinnütziger Organisationen, unter denen wiederum die Internet-Beratung der TelefonSeelsorge positiv auffällt.

Das Online-Beratungs-Angebot der TelefonSeelsorge wird im Jahr 1995 von Frank Well und Kollegen aus den Niederlassungen Köln und Krefeld ins Leben gerufen und erweist sich als so erfolgreich, dass im Jahr 2000 ein bundesweites Projekt daraus wird (vgl. van Well, 2000; Bieber-Eckard, 2006). Anlass für den Ausbau des Projektes war die enorme Resonanz, die in der Anfangsphase verzeichnet werden konnte: Innerhalb eines Jahres von 1999 bis 2000 hat sich die Nachfrage von 2.500 auf 5.100 mehr als verdoppelt (Bieber-Eckardt, 2006). Die bei der TelefonSeelsorge beschäftigten Berater sind entweder angestellte Fachkräfte (Psychologen und Sozialarbeiter) oder ehrenamtliche Mitarbeiter, die eine Ausbildung in der Telefonberatung erhalten haben.

Die Kontaktaufnahme erfolgt durch meist relativ kurze E-Mails, in denen die Ratsuchenden ihre Situation darstellen und die innerhalb von 24 Stunden bearbeitet werden. „Die Arbeit in einer ersten Antwort-Mail besteht (...) vor allem darin, sich selbst vorzustellen, Vertrauen aufzubauen und zu zeigen, daß man sein Gegenüber ernst nimmt, ihn versteht und auf ihn eingeht. Die wesentliche Technik besteht darin, zusammenzufassen, zu spiegeln und auf Zusammenhänge hinzuweisen, die dem Betroffenen selbst eventuell nicht aufgefallen sind“, schreibt Christl (1998, S. 110), einer der Begründer des Internet-Beratungsangebotes, der sich seit deren Entstehung auch wissenschaftlich mit dem Thema befasst hat. In etwa der Hälfte der Fälle bleibt es bei diesem Einmalkontakt, andernfalls wird die Beratung so lange fortgesetzt, bis entweder eine positive Wende eintritt oder der Ratsuchende sich an einen niedergelassen Berater oder Therapeuten wendet. Eine zeitliche Begrenzung für das Beratungsangebot gibt es nicht. Bei solchen längerfristig angelegten Beratungen erhält der 
Hilfesuchende einmal wöchentlich eine Antwort auf seine E-Mails, wobei der Berater seine Anmerkungen direkt in die vom Ratsuchenden gesendete Nachricht hineinschreibt, so dass eine „quasi-dialogische Struktur“ (van Well, 2000, S. 10) entsteht.

Die zweite Form der Online-Beratung wird von den Ratsuchenden wesentlich seltener gewählt: nur 16,6\% entscheiden sich für einen Zweier-Chat mit dem Berater. Für die Bevorzugung des E-Mail-Kontaktes lassen sich im Wesentlichen zwei Gründe anführen: zum einen sind beim Chatten Überschneidungen und Verzögerungen im Austausch - zum Beispiel aufgrund technischer Schwierigkeiten wahrscheinlicher als beim E-Mail-Kontakt. Zum anderen ist der Ratsuchende beim Verfassen von E-Mails ungebundener, d.h. er kann sie zu jeder beliebeigen Tageszeit abschicken ohne sich an einen verabredeten Termin halten zu müssen, und es besteht die Möglichkeit, die E-Mails nach dem Verfassen noch einmal zu bearbeiten und zu revidieren. So kann der Inhalt eher kontrolliert werden als beim spontanen Austausch im Chat. Dies ist für die meisten Nutzer von großer Wichtigkeit, da Menschen, die im Internet Rat suchen, oft ein besonderes Kontrollbedürfnis haben und daher diese niedrigschwellige Kommunikationsform wählen (vgl. Christl, 1998, 2000; van Well, 2000). 


\section{Eigenes Projekt}

Das Projekt trägt den Namen OPIS (Online-Patienten-Informations-Service) und wurde offiziell im Juli 2004 durch Gumpert und Jungermann ins Leben gerufen. Der Autor dieser Arbeit wirkt für den Bereich Psychiatrie und Psychotherapie seit ca. 2 Jahren mit.

\section{Vorgeschichte und Hintergründe:}

Bereits in seiner Weiterbildung zum Facharzt für Orthopädie im Jahre 2002 fiel Gumpert ein enormes Defizit in den Möglichkeiten der angemessenen Informationsvermittlung gegenüber dem Patienten auf. Oftmals gestaltete sich die persönliche Informationsvermittlung aus Zeitnot als zu knapp.

In den typischen assistenzärztlichen Funktionen wie Stationsversorgung, operative Maßnahmen, sowie poststationäre Versorgung blieb auch dem engagiertesten Assistenten häufig zu wenig Zeit auf die (oftmals immer gleichen) Fragen der Patienten angemessen einzugehen.

Dies führt im weiteren dazu, dass Dr. Gumpert begann diese typischen „FAQ“'s (Frequently asked questions) in Form von leicht bzw. laienverständlichen Texten aufzuschreiben und diese auf einer eigene Homepage kostenlos zur Verfügung zu stellen.

Der Grundsatz der Seite war (und ist auch heute), dass nur ein aufgeklärter Patient durch Verständnis seiner Erkrankung und der Therapiemöglichkeiten, einen optimalen Therapieerfolg erzielen wird. Hierzu soll durch laienverständliche Darstellung von Ursachen, Ätiologien und Therapieoptionen eine Transparenz geschaffen werden, aus der eine Kompetenz für die aktive Therapiemitgestaltung entsteht.

Diese Auffassung wurde offensichtlich von vielen Patienten geteilt, da die Besucherzahlen ständig stiegen und auch die sog. "Google-Platzierungen“ stetig besser wurden. Eine „Google-Platzierung“ beschreibt den Rang, auf dem ein Suchbegriff bei dieser Suchmaschine aufgeführt wird.

Über 80\% aller Menschen, die im Internet etwas suchen verwenden in Deutschland Google als Suchmaschine. (Anmerkung: In den USA z.B. hat Yahoo einen deutlich höheren Marktanteil) Wenn man darüber hinaus zu Gute hält, dass sich die ersten 3 Platzierungen etwa 
70\% der Besucher „teilen“, ist es um so beeindruckender, dass bereits Ende 2005 über 100 orthopädische Suchbegriffe von OPIS auf Platz 1 gelistet waren.

\section{Die ursprünglichen Säulen von OPIS}

Die Grundidee des Online-Patienten-Informations-Service war, die Informationsvermittlung. Weil die Besucherzahlen jedoch in einem hohen Maße wuchsen, wurde schon bald eine 2. Säule notwendig. Im Vorfeld kam es zu immer mehr persönlichen E-Mail-Anfragen, welche die Verantwortlichen der Seite schon recht bald an die Grenze des Leistbaren führte. Aus diesem Grunde wurde die Installation eines Forums notwendig, in dem Patienten Fragen stellen konnten. Hier kam es dann zu einer Art ,ärztlichen Sprechstunde“ durch die Betreiber der Seite.

Darüber hinaus war es den Betreibern nach einiger Zeit möglich „kompetente Laien“, also regelmäßige Forumsbesucher, die sich durch ein kompetentes Wissen und freundlichen Umgang hervorgetan hatten, als Moderatoren einzusetzen, um ,nicht-ärztliche“ Dinge zu beantworten und somit die Administratoren zu entlasten.

Aus der Notwendigkeit heraus, dass die zur Verfügung gestellten Informationen offensichtlich den ungeheuren Informationsbedarf nur ungenügend befriedigen konnten entstand letztendlich die 3. Säule von OPIS.

Die Betreiber der Seite begannen Informationsbücher zu verfassen. Diese unterschieden sich im Umfang deutlich von den Online-Texten. Hat ein durchschnittliches Online-Thema etwa einen Umfang von 5 -9 Seiten, beschäftigen sich die Informationsbücher auf etwa 50-100 Seiten mit der jeweiligen Thematik. Da sich im Weiteren die Besucherzahlen stetig weiter nach oben entwickelten, entschlossen sich Gumpert und Jungermann schließlich zur Gründung einer Gesellschaft bürgerlichen Rechts.

\section{Das Informationssystem OPIS und die Psychotherapie}

Da sich im Zuge der Entwicklung von der Internetpräsentation scheinbar ungeahnte Expansionsmöglichkeiten boten, war es nur logisch, dass die Betreiber der Seite nach ständigen Optimierungsmöglichkeiten suchten. Dies lag zunächst in einer nahezu 
vollständigen Abdeckung orthopädischer Themen einschließlich seltener Randthemen, sowie in der Verfassung von 17 Informationsbüchern und der weiteren „Google-Optimierung“.

Schnell waren jedoch 2 Dinge klar:

1. Die Optimierungsmöglichkeiten bei nur einem Thema (Orthopädie) waren begrenzt und somit endlich.

2. Informationsbedarf besteht in nahezu allen anderen Fächern der Medizin.

Somit wurden Anfang 2005 mit dem Autor erste Gespräche geführt, um einen „psychiatrischen Testballon“ steigen zu lassen.

Ich befand mich zu diesem Zeitpunkt im 4. Jahr meiner psychiatrischen Weiterbildung in einer psychiatrischen Universitätsklinik und kannte somit die oben bereits aufgeführten Defizite in der Informationsvermittlung und den daraus resultierenden „Wissenshunger“ recht gut.

Im Mai 2005 ging somit das erste psychiatrische Thema (Depression) online und OPIS hatte somit sein Angebot auf eine zweite medizinische Fachrichtung erweitert.

Da die ursprüngliche OPIS-Seite bereits ein gut laufendes und weitestgehend optimiertes System darstellte, wurde der Einstieg somit relativ leicht, so dass ich die bereits bewährten 1 . und 2. Säulen (Online-Texte und Forum mit ärztlicher Betreuung) übernahm. Im weiteren Verlauf kamen dann auch noch einige Informationsbücher hinzu.

Neu hinzu kamen für den psychiatrische-psychotherapeutischen „Flügel“ der Seite eine eigens produzierte CD mit Übungen zur progressiven Muskelentspannung nach Jakobson (ein etabliertes psychotherapeutisches Entspannungsverfahren), sowie eine selbst produzierte CD mit Traumreisen (eine weitere psychotherapeutische Methode).

\section{Online Beratung und Telefonkontakte:}

Im Mai 2006 verließ ich nach Vollendigung meiner Weiterbildungszeit das Krankenhaus und begann eine ärztliche Tätigkeit in einer niedergelassenen nervenärztlichen Praxis. In diesem Setting bot sich noch einmal eine völlig neue Qualität dessen, was in unserem Gesundheitssystem nicht stimmt. 
Aufgrund eigener Erfahrungen im niedergelassenen Bereich, ist mir bekannt, dass es, um wirtschaftlich arbeiten zu können, notwendig ist etwa 40-50 Patientengespräche pro Tag zu führen. Hieraus ergibt sich eine Durchschnittszeit von 7-10 Minuten pro Patient. Hinzu kommen hierbei teilweise grotesk hohe Wartezeiten die zwischen 2 Monaten (neurologisch) bis zu 12 Monaten (psychotherapeutisch) liegen.

Aus dieser Situation heraus wurde schließlich die Idee einer Online- bzw. Telefonberatung heraus geboren.

Ein solches fachärztliches Angebot ist (meiner Kenntnis nach) bislang einzigartig. Bei der Onlineberatung nimmt der Patient per E-Mail Kontakt auf und schildert auf einer definierten Seitenanzahl sein Grundproblem. Er bekommt dann eine kurze Bestätigung und eine Zahlungsanweisung von $40 €$ und die Gelegenheit mir eine 2.Mail mit weiterführenden Erklärungen und Erläuterungen zu schicken. Nach Geldeingang geht ihm dann die von mir verfasste E-Mail (Umfang ca. 2-3 Seiten) zu.

Beim Telefonkontakt hat der Patient die Möglichkeit Zeiteinheiten (20 Minuten) zu erwerben. Anschließend wird ein Telefontermin ausgemacht und ich rufe den Patienten dann an.

Insgesamt handelt es sich bei den Beratungsangeboten nicht um klassische psychotherapeutische Kontakte, sondern um tatsächliche konkrete Beratung zu einzelnen Fragen bezüglich Diagnose, therapeutischer Maßnahme oder auch Nebenwirkungen von Psychopharmaka. Die Kontakte stellen somit für die Patienten eine Art „,anoymisierten Arztbesuch“ dar, bei dem sie ausführlichst Gelegenheit bekommen sich auszudrücken und bei dem sie eine ausführliche und befriedigende Antwort erhalten.

\section{Online-Therapie}

Neben den zahlreichen Beratungsangeboten, gibt es auch Ansätze für internetbasiert Psychotherapie. Im Folgenden werden drei solcher Konzepte vorgestellt: das erste hauptsächlich aus historischen Gründen, da es eines der ersten Konzepte für Online-Therapie war. Die beiden weiteren Konzepte wurden wegen ihrer Aktualität, wissenschaftlichen Fundierung und Verfügbarkeit im deutschen Sprachraum ausgewählt. 


\subsection{Therap-e-mail}

Einer der ersten Vorstöße in Richtung internetbasierter Psychotherapie wurde im Jahr 1998 von Murphy \& Mitchell gemacht, die ihr Konzept als „Therap-e-mail“ bezeichnen. Wie der Name schon sagt, basiert die Kommunikation zwischen Therapeut und Patient auf dem Austausch von E-Mails. Der erste Schritt des Therapeuten - nach der Kontaktaufnahme von Sei-

ten des Klienten - besteht in einer herzlichen Begrüßung, die eine Vertrauens- und Sympathiebasis für die weitere Arbeit schaffen soll. Danach muss der Patient einen standardisierten Fragebogen (Virtually Solve It worksheet = VSI) bearbeiten, anhand dessen der Therapeut Erkenntnisse über das Verhalten des Patienten sowie sein Problem gewinnt. Darüber hinaus dient die Bearbeitung auch dem Erkenntnisgewinn des Patienten und ist somit nicht Vorstufe der Therapie, sondern bereits ein Teil derselben. Die Autoren formulieren dies folgendermaßen: „, In fact, we wondered whether the VSI might be, for some clients, a catalyst for change that may not require further therapeutic support“ (Murphy \& Mitchell, 1998, S. 22). Im anschließenden E-Mail-Austausch ist der Therapeut darum bemüht, dem Patienten „,durch diverse Nachfragen (Anregungen für) eine genaue Analyse des eigenen Verhaltens und Erlebens (...), (und ihn) durch Interpretationsvorschläge zum Umdenken anzuregen und positive Veränderungsansätze zu bekräftigen“ (Döring, 2000, S. 540). Das Vorgehen ist also ähnlich zu dem von van Well beschriebenen bei der Online-Beratung der Telefonseelsorge.

Die von Short et al. (1976) in der Social Presence Theory geäußerten Zweifel daran, ob bei computervermittelter und textbasierter Kommunikation ein Gefühl der Nähe entstehen kann, weisen Murphy \& Mitchell mit einem Hinweis auf frühere Generationen als unbegründet ab. $\mathrm{Zu}$ einer Zeit, als Videokonferenzen und Telefon noch ebenso undenkbar waren wie Flugreisen waren die Menschen darauf angewiesen, den Kontakt zu entfernt lebenden Personen mittels Briefen aufrecht zu erhalten. Die in Erfahrungsberichten geschilderten Wirkungen dieser Briefe sehen Murphy und Mitchell als unwiderlegbares Zeichen dafür, dass Wärme, Nähe und Mitgefühl auch in Textnachrichten übermittelt werden können. Darüber hinaus führen sie positives Feedback, das sie von früheren Klienten erhalten haben, als Beleg für die Wirksamkeit der Online-Therapie an und weisen darauf hin, dass computervermittelte Kommunikation dem Face-to-Face-Dialog in mancher Hinsicht sogar überlegen ist: durch die Digitalisierung und die damit einhergehenden Möglichkeiten zur Datenspeicherung ist der Klient in der Lage, die Anregungen des Therapeuten immer wieder nachzulesen und kann den Behandlungsverlauf anhand der gespeicherten Nachrichten nachvollziehen. 
Die Datenspeicherung ist aber nicht nur für den Patienten von Vorteil, sondern erleichtert auch die Supervision, da der Gesprächsverlauf objektiv nachvollzogen werden kann.

\subsection{Interapy}

Ein anderes Konzept der Online-Therapie wurde in den Niederlanden von Lange und Kollegen (Lange, Schrieken, van de Ven, Bredeweg, Emmelkamp, van der Kolk, Lydsdottir, Massaro \& Reuvers, 2000) entwickelt und nennt sich Interapy.

Entwickelt wurde Interapy nachdem sich in einer Untersuchung, dem sog. „Amsterdam Writing Project“ (Schoutrop, Lange, Brosschot \& Everaerd, 1997; Schoutrop, 2000), der positive Effekt strukturierten Schreibens auf Posttraumatische Belastungsstörungen (PTBS) gezeigt hatte. In der Anfangsphase war auch die Interapy lediglich zur Behandlung von PTBS gedacht und hat sich bei der Behandlung derselben auch bewähren können (Lange, van de Ven, Schrieken \& Emmelkamp, 2001; Lange, Rietdijk, Hudcovicova, van de Ven, Schrieken, \& Emmelkamp, 2003). Mittlerweile gibt es auch Äquivalente zur Behandlung des Burn-OutSyndroms (Lange, van de Ven, Schrieken \& Smit, 2004) und von Bulimie. In den Niederlanden ist Interapy bereits ein anerkanntes Therapie-Konzept, deutsche Entsprechungen stecken noch in der Testphase. Die Kosten für eine Therapie belaufen sich auf 1250 EUR.

Es handelt sich bei Interapy um eine protokollbasierte Schreibtherapie; der Kontakt zwischen Therapeut und Patient basiert ausschließlich auf dem Austausch von E-Mails und folgt einem festgelegten Ablaufschema, das sich in folgende Behandlungsphasen unterteilen lässt.

\section{(1) Aufklärung}

Die erste Behandlungsphase verfolgt das Ziel die Patienten über das typische Symptombild der betreffenden Störung aufzuklären. Außerdem werden ihnen nützliche Literatur-Tipps und Informationen zum Behandlungsverlauf mitgeteilt.

(2) Diagnose

Der zweite Schritt besteht darin, dass mittels standardisierter Fragebögen eine Diagnose erstellt und die Eignung des Patienten für die Interapy überprüft wird. Unter anderem gelten Substanzmissbrauch und Komorbiditäten als Ausschlusskriterium.

(3) Behandlung 
In der dritten Phase findet die eigentliche Behandlung statt. Im Laufe dieser Phase verfassen die Patienten insgesamt 10 Texte (2 / Woche; je 45 Minuten); nach jedem zweiten Artikel gibt der Therapeut ein Feedback. Diese Behandlungsphase dauert also fünf Wochen und lässt sich wiederum in drei Abschnitte unterteilen.

a) Selbstkonfrontation

Die Patienten müssen in vier Texten ihr traumatisches Erlebnis möglichst genau beschreiben. Dieser Konfrontation mit dem Geschehenen wird per se schon therapeutische Wirkung zugeschrieben.

b) Kognitive Umstrukturierung

In vier weiteren Texten müssen die Patienten die Perspektive wechseln und einem fiktiven Opfer Ratschläge zur Bewältigung des Erlebten erteilen.

c) Abschied

Zum Abschluss werden noch einmal zwei Texte geschrieben, die zusammen einen Brief bilden, der nach Beendigung der Behandlung abgeschickt werden kann, aber nicht muss. Die Vergangenheit wird mit dem Brief abgeschlossen, wodurch ihr ein neuer Platz zugewiesen und ihr Einfluss auf die Gegenwart somit verringert wird.

(4) Follow-Up

Eine Nachfolgeuntersuchung sechs Wochen nach Beendung der Therapie soll mit Hilfe standardisierter Fragebögen, die denen in Phase 2 ähnlich sind, der Behandlungserfolg überprüft werden.

\subsection{Theratalk}

Ein Online-Therapie-Konzept, das im deutschsprachigen Raum entwickelt wurde, ist Theratalk, ein Projekt des Psychologischen Instituts der Universität Göttingen. Theratalk wurde im Jahr 1996 ins Leben gerufen und kann dank seines wissenschaftlichen Hintergrundes ein fundiertes und erprobtes Konzept vorweisen. Untersuchungen konnten belegen, dass sie Wirksamkeit der Online-Therapie via Theratalk sich nicht signifikant von der einer Face-to-Face-Therapie unterscheidet (vgl. Beer, 2001; Beer \& Breuer, 2004).

Dieses Angebot ist auf Paartherapie, Paarberatung und Eheberatung spezialisiert. Es werden vier Abstufungen der Unterstützung unterschieden.

(1) Information

Die Ratsuchenden haben die Möglichkeit, sich mit einem kostenlosen Video-Podcast über aktuelle Erkenntnisse der Partnerschaftsforschung sowie deren praktische Umsetzungen zu informieren. 
(2) Bestandsaufnahme

Wer sich unsicher ist, ob er Unterstützung für seine Partnerschaft braucht, kann diese in einem kostenlosen Partnerschaftstest einer Bestandaufnahme unterziehen und so herausfinden, ob weitergehende Hilfe notwendig ist.

(3) Unkomplizierte Unterstützung

Eine Form der Unterstützung ist das Ressourcen-Aktivierungs-System (R.A.S.), das verschiedene Module enthält mit Hilfe derer beispielsweise das Anerkennen der Wünsche des Partners eingeübt werden kann. Diese Form der Hilfe beinhaltet noch keinen Therapeutenkontakt.

(4) Umfangreiche Hilfe

Den höchsten Grad der Unterstützung stellt die eigentliche Paarberatung oder Paartherapie dar. Die Kommunikation zwischen Paar und Therapeut erfolgt in Chat-Sitzungen; zusätzlich treten die Partner einzeln mit dem Therapeuten in Kontakt (per Chat oder EMail). Angelegt ist die Therapie auf eine Gesamtdauer von 6 Wochen, wobei aber bei Bedarf die Option auf Verlängerung besteht. Die Kosten betragen 100 EUR/ Woche. In den ersten beiden Wochen der Therapie werden der Status der Paarbeziehung und die Wünsche die Partner erfasst. In den folgenden Wochen werden die Ziele expliziert und umgesetzt. Der Therapeut gibt den Ratsuchenden Übungen, die sie zu Hause durchführen sollen. In der gesamten Zeit der Behandlung haben die Paare an jedem Werktag Kontakt mit dem Therapeuten.

\subsection{Von der internetbasierten zur mobilen Intervention}

Neben den oben beschriebenen Formen der internetbasierten Intervention wurden in der jüngsten Vergangenheit auch die Einsatzmöglichkeiten von mobilen Kommunikationsmitteln für die psychologische Beratung und Psychotherapie diskutiert.

Unter mobilen Kommunikationsmitteln bzw. Mobilmedien werden dabei nicht nur Handys verstanden, sondern auch portable Spielkonsolen, Handhelds und Laptops.

Der Vorteil solcher Medien ist in deren flexiblen Einsatzmöglichkeiten zu sehen. Darüber hinaus werden sie von größeren Teilen der Gesellschaft genutzt als das Internet. Über $90 \%$ der jungen Menschen sind per Handy erreichbar, die Zahl der Mobiltelefone in Deutschland übersteigt sogar die Bevölkerungszahl. 
Beispielsweise im Bereich der medizinischen Ausbildung werden Mobilmedien bereits seit längerem genutzt (Johnston, Leung, Tin et al., 2004); dies wurde unter dem Namen mlearning (mobile learning) bekannt.

Der Einsatz von Mobilmedien für eine therapeutische Intervention kann an drei Stellen der Behandlung erfolgen (s. Döring \& Eichenberg, 2007):

$\S$ Vor Beginn der eigentlichen Intervention zur Diagnose und zur Klärung der Frage, welche Maßnahmen geeignet sind

$\S \quad$ zur Intervention im engeren Sinn

$\S$ Nach Abschluss der eigentlichen Intervention zur Erfolgsbewertung.

Die Bandbreite an Angeboten zur mobilen Therapie ist mindestens ebenso groß wie die zur internetbasierten Therapie. Eine ausführliche Darstellung würde daher den Rahmen dieser Arbeit sprengen. Im Folgenden soll daher nur ein kurzer Überblick gegeben werden (nach Döring \& Eichenberg, 2007; eine detaillierte Beschreibung findet sich auch bei Istepanian, Laxminarayan \& Pattichis, 2006).

$\S$ Handydiagnose

Der Einsatz von Handys zu diagnostischen Zwecken ist auf zwei Arten denkbar: zum einen können Patienten Selbstauskünfte zu ihrem Befinden (z.B. über eine SMS-Abfrage), zum anderen ist auch eine Erfassung physiologischer Daten - mit Hilfe eines speziellen Gerätes (z. B. vitaphone) möglich. Die Handydiagnose kann lediglich als Ergänzung nicht als Ersatz einer herkömmlichen Diagnostik und Behandlung gesehen werden.

\section{$\S \quad$ SMS-Beratung}

Gemeinnützige Einrichtungen wie etwa Pro Familia bieten ergänzend zur Telefonberatung auch die Möglichkeit zur SMS-Beratung, die insbesondere zur ersten Kontaktaufnahme und zur Weitervermittlung geeignet ist. Mit Hilfe spezieller Software können die ausgetauschten Textnachrichten auf den Computer übertragen und so archiviert werden. Diese Form der Beratung wird insbesondere von Jugendlichen in Anspruch genommen, kann jedoch bei schweren Krisen nicht ausreichend sein und sollte in einem solchen Fall durch eine telefonische, internetbasierte oder herkömmlich Beratung ersetzt oder ergänzt werden.

\section{$\S$ SMS-Therapie}

Eine Therapie, die sich ausschließlich auf SMS-Kontakt begrenzt, ist - u. a. aufgrund der Kürze der Nachrichten - nicht angebracht. Der Austausch von Textnachrichten kann jedoch beispielsweise nach Abschluss einer stationären Behandlung ergänzend hinzukommen und den Übergang in den Alltag erleichtern. An anderer Stelle in dieser 
Arbeit wird eine Studie vorgestellt (Kap. 5.2.4), die die Wirksamkeit einer solchen SMSBrücke in der Nachbehandlung von Bulimie-Patienten untersucht.

$\S$ Mobiltelefonietherapie

Mobiltelefone können in der Angsttherapie zur Unterstützung der Patienten bei in vivoKonfrontationen mit dem anxiogenen Objekt eingesetzt werden. Auf diese Weise sind die Patienten flexibler im Einüben solcher Situationen, es muss jedoch darauf geachtet werden, dass sich keine Abhängigkeit von der telefonischen Unterstützung entwickelt.

$\S$ Handhelddiagnose

Ähnlich wie per Handy können auch per Handheld (z.B. Palm-Pilot) Selbstreportdaten und physiologische Parameter erfasst werden; mit dem Unterschied, dass ein Handheld umfangreichere Datenerhebungen möglich macht. Das Projekt „Lifechart“ setzt Handhelddiagnose bei der Behandlung bipolarer Störungen ein. Die erhobenen Daten werden zentral gespeichert, grafisch aufbereitet und sowohl dem Patienten als Feedback rückgemeldet, als auch für die Forschung zur Verfügung gestellt. Wie auch die Handydiagnose kann die Handhelddiagnose herkömmliche Verfahren allenfalls ergänzen, nicht aber ersetzen.

$\S$ Handheldrehabilitation

Handhelds werden insbesondere im Bereich der Gedächtnis- und Orientierungsstörungen zur Rehabilitation eingesetzt. Mit Hilfe spezieller Systeme können die Patienten per Hansheld an Termine erinnert werden, Checklisten zum Tagesablauf erstellen oder durch Fotos die Zuordnung von Namen und Personen einüben. Auf diese Weise kann Handheldrehabilitation dazu beitragen, dass die Betroffenen selbständiger im Alltag agieren können und der Betreuungsaufwand so reduziert werden kann. Auch der Einsatz in der Behandlung von Patienten mit Asperger-Syndrom oder Sprachstörungen ist möglich.

\section{$\S$ Handheldtherapie}

Konzepte zur Therapie mit Hilfe des Handhelds stammen überwiegend aus dem Bereich der Verhaltenstherapie und sind lediglich als Ergänzung einer Face-to-Face-Behandlung gedacht. So können beispielsweise physiologische Maße von Patienten mit Panikstörungen erfasst werden oder Menschen mit Sozialphobie in situ Selbstauskünfte bezüglich der Angstausprägung aufzeichnen. 
Interventionsansätze, die das Notebook als Mobilmedium einbinden, werden hier nicht gesondert vorgestellt, da diese im Wesentlichen das Spektrum internetbasierter Behandlungsmöglichkeiten (s. o.) umfassen.

Möglichkeiten und Grenzen von internetbasierter und mobiler Therapie sind analog zu bewerten: Unterschiede bezüglich der Flexibilität des Einsatzes sowie der Länge der ausgetauschten Nachrichten bzw. erfragten Informationen, sind zwar zu beachten, generell gelten aber die Vor- und Nachteile computervermittelter Kommunikation auch für die Kommunikation über Mobilmedien.

„Ebenso wie Onlinemedien haben auch Mobilmedien potenziell sowohl salutogene als auch pathogene Potenziale: Mobilmedien können nicht nur sozialverträglich, psychologisch unterstützend und heilend wirken, sondern - je nach Nutzungsweise und Nutzungskontext - auch vorhandene Störungen verstärken oder neue Störungen entstehen lassen“

(Döring \& Eichenberg, 2007).

\section{Empirische Befunde zur Online-Therapie}

An dieser Stelle sollen die bisher erschienen wichtigen Wirksamkeitsstudien zur OnlineTherapie dargestellt werden. Zuerst wird eine Zusammenfassung der von Ott (2003) berichteten Ergebnisse gegeben. Im Anschluss daran werden ausgewählte Studien zum Thema vorgestellt: dies sind zwei Studien zur Wirksamkeit von internetbasierten TherapieKonzepten, sowie zwei Studien, die Kombinationsmöglichkeiten von Face-to-Face-Therapie und computervermittelter Kommunikation bzw. textbasierter Kommunikation via Handy untersuchen.

Die empirischen Befunde sollen Belegen, dass es eine echte Diskrepanz zwischen den Theorien computervermittelter Kommunikation und der tatsächlichen Wirksamkeit von Online-Therapie gibt.

\section{1 Überblick über die bisherige Forschung}

Ott (2003) hat eine umfangreiche Literaturrecherche zu klinisch-psychologischen Intervention im Internet vorgelegt. Ziel des Reviews war es, die bis dato erschienen empirischen Studien zur Wirksamkeit internetbasierter Intervention (IBI) zu sammeln, nach Vorgehen und Art der behandelten Störung zu klassifizieren und so einen Überblick über den Stand der Forschung zu geben. Abbildung 7 gibt einen Überblick über das Vorgehen des Autors. 


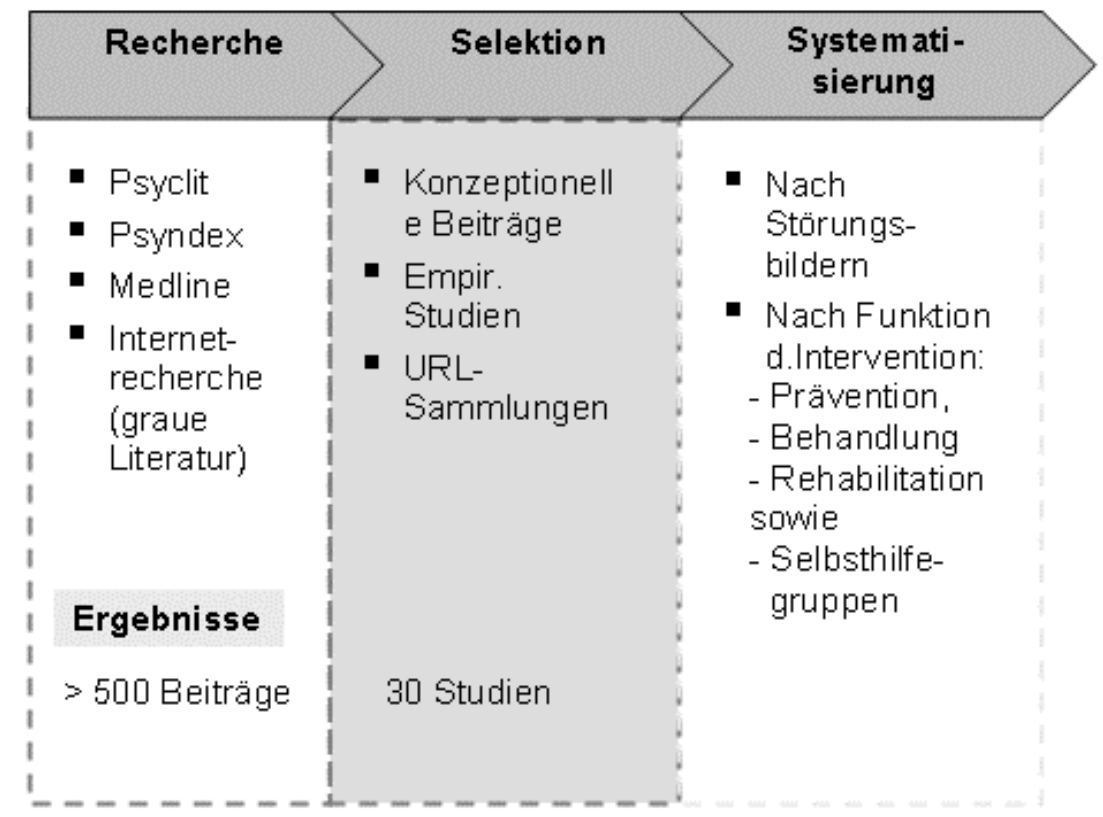

Abb. 7: Vorgehensweise bei der Literaturanalyse (Ott, 2003, S. 130)

Die 30 Studien, die schließlich in die Analyse eingingen, unterschieden sich bezüglich verschiedener Kriterien. Zum einen wurden unterschiedliche Störungen behandelt (vgl. Abb.8), zum anderen wurden mit der Intervention unterschiedliche Zielsetzungen verfolgt: ,in über der Hälfte der Studien wurden IBI Verfahren zur Behandlung eingesetzt, die restlichen Studien verteilen sich gleichmäßig auf die Interventionsfunktionen Prävention und Rehabilitation (jeweils 23,3\%)“"(Ott, 2003, S. 132). Auch hinsichtlich der theoretischen Ausrichtung der Behandlung unterschieden sich die Studien: die überwiegende Mehrheit der Studien setzte zwar auf kognitiv-behaviorale Verfahren, aber auch rein verhaltenstherapeutische, edukative und klientenzentrierte Methoden wurden eingesetzt. Eine geringe Anzahl an Untersuchungen verwendete schließlich Verfahren, die keine theoretischen Grundlagen besaßen oder zumindest diese nicht explizierten.

Ott untersuchte die Wirksamkeit der Interventionen, wobei eine Behandlung dann als erfolgreich galt, wenn die Symptomatik nach der Intervention sich signifikant verbessert hatte im Vergleich zur Symptomausprägung bei Behandlungsbeginn. Manche Studien haben nicht nur eine Experimentalgruppe erhoben, sondern zusätzlich auch eine Kontrollgruppe. Dabei kann es sich entweder um eine Wartekontrollgruppe handeln, oder eine Kontrollgruppe, die sich einer Alternativbehandlung - in diesem Fall einer Therapie im herkömmlichen Face-toFace-Setting - unterzogen hat. Ein Behandlungserfolg im Fall einer Untersuchung mit 
Wartekontrollgruppe wäre dann gegeben, wenn sich die Symptomatik der behandelten Gruppe zum Ab-

schluss der Behandlung signifikant von der der Wartekontrollgruppe unterschiede - bei gleichen Werten zur Studienbeginn. Beim Vergleich mit einer Gruppe, die eine herkömmliche Therapie erhalten hatte, wurde sowohl eine Überlegenheit der Experimentalgruppe als auch eine Übereinstimmung der Effekte als Erfolg gewertet, da die Wirksamkeit der herkömmlichen Face-to-Face-Therapie vorausgesetzt wurde.

Insgesamt zeigt sich so ein sehr positives Bild: 26 der 30 in die Analyse aufgenommenen Studien (also 86,7\%) konnten positive Effekte nachweisen. Wirksamkeitsunterschiede sind weniger auf die verwendeten Verfahren zurückzuführen als auf das Störungsbild: besonders von internetbasierten Interventionen $\mathrm{zu}$ profitieren scheinen Personen, die unter Panikstörungen, posttraumatischen Belastungsstörungen, Essstörungen oder Suchtproblemen leiden. Depressive Störungsbilder scheinen von einer internetbasierten Intervention weniger $\mathrm{zu}$ profitieren. Abbildung 8 zeigt eine Übersicht über die Anzahl der erfolgreichen Behandlungen mit IBI-Verfahren im Vergleich mit der Gesamtzahl der durchgeführten Studien, wobei hier nach den theoretischen Grundlagen der eingesetzten Verfahren getrennt wurde.

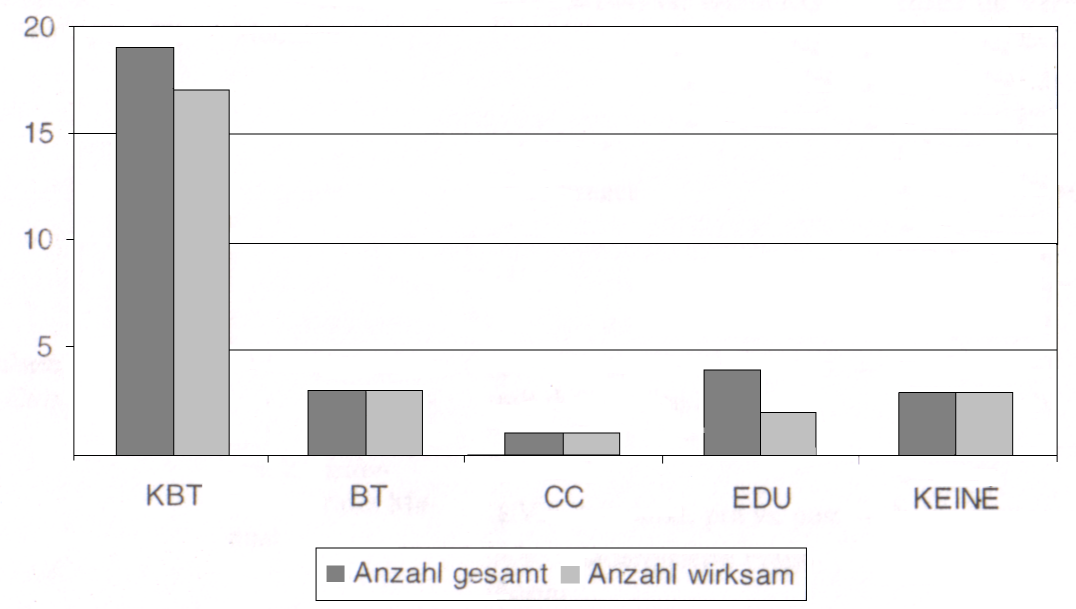

Abb. 8: Anzahl der Studien insgesamt und der wirksamen Studien, getrennt nach ihren theoretischen Grundlagen $. \mathrm{KBT}=$ kognitiv-behavioral, $\mathrm{BT}=$ verhaltenstherapeutisch, $\mathrm{CC}=$ klientenzentriert, EDU = edukative Maßnahmen. (nach Ott, 2003, S. 132)

Der durchaus positive Befund der Literaturanalyse darf aber nicht darüber hinwegtäuschen, dass die Gesamtzahl an Wirksamkeitsstudien, die zum Zeitpunkt der Untersuchung vorlagen sehr gering ist und einige Lücken und Verzerrungen aufweist; dies betrifft sowohl die 
behandelten Störungsformen als auch die theoretische Ausrichtung der verwendeten Verfahren. So lagen zum Zeitpunkt der Analyse noch keinerlei Untersuchungen zur Behandelbarkeit von Zwangsstörungen, somatoformen Störungen, Schizophrenien und Persönlichkeitsstörungen vor. Dies wird nicht nur von Ott (2003) kritisiert, sondern beispielsweise auch von Taylor \& Luce (2003).

Hinsichtlich der verwendeten Verfahren zeigt die Datenlage eine deutliche Verzerrung zu Gunsten kognitiv-behavioraler Ansätze. „Klientenzentrierte und tiefenpsychologische Ansätze bestehen bis dato zum größten Teil nur aus konzeptionellen Überlegungen zum generellen Einsatz neuer Informationstechnologien (bspw. Galanter \& Brook, 2001, für klientenzentriertes IBI für Alkoholismus)“ (Ott, 2003, 141). Lediglich bei einer der 30 Wirksamkeitsstudien (Woodruff, Edwards, Conway \& Elliott, 2001) kamen klientenzentrierte Verfahren zum Einsatz.

Das Ziel zukünftiger Forschungen in diesem Bereich sollte die Abdeckung der bislang noch bestehenden Lücken sein. Darüber hinaus wären Studien wünschenswert, die nicht nur eine Kontrollgruppe erheben, sondern sowohl eine Wartekontrollgruppe als auch eine alternativ (Face-to-Face) behandelte Kontrollgruppe, nur so können fundierte Aussagen über die Wirksamkeit von internetbasierten Therapieformen gemacht werden. Die zunehmende Popularität des Themas sowie das wachsende Forschungsinteresse lassen aber hoffen, dass die Zahl der Studien zu diesem Thema in der Zukunft ansteigen wird.

\subsection{Ausgewählte Studien}

Nachdem nun ein Überblick über den Stand der Forschung bis zum Jahr 2003 gegeben worden ist, sollen im Folgenden ausgewählte Wirksamkeitsuntersuchungen zur internetbasierten Therapie vorgestellt werden. Dabei sollen insbesondere solche Studien berücksichtigt werden, die nach der oben geschilderten Literaturanalyse durchgeführt wurden. Als erstes wird eine Evaluation des Interapy-Konzeptes vorgestellt, danach wird auf eine Wirksamkeitsstudie zum Theratalk-Konzept eingegangen.

Beide Untersuchungen erweisen sich auch deswegen als interessant, weil sie sich mit deutschsprachigen Internet-Therapie-Projekten beschäftigen, wodurch sie sich von der Mehrzahl der von Ott angeführten Studien unterschieden. 


\subsubsection{Behandlung von Posttraumatischen Belastungsstörungen mit dem Interapy- Verfahren}

Das Interapy-Behandlungsprotokoll wurde von seinem Entwickler Alfred Lange von der Universität Amsterdam sowie zwei Wissenschaftlern der Universität Zürich, Dipl.-Psych. Christine Knaevelsrud und Prof. Dr. Dr. Andreas Maercker ins Deutsche übertragen und in einer dreijährigen Untersuchung an Personen mit posttraumatischen Belastungsstörungen evaluiert. Diese Untersuchung wurde vom Weißen Ring e.V., einer Hilfsorganisation für Kriminalitätsopfer, unterstützt und ist Gegenstand zweier Publikationen (Knaevelsrud, 2005; Maercker, 2005), die auch die Basis der Ergebnisschilderung hier sind.

Das Vorgehen bei der Interapy wird an dieser Stelle nicht mehr geschildert, da es bereits an anderer Stelle (Kap. 3.5.2) ausreichend beschrieben wurde.

An einem ersten Screening nahmen 520 Personen teil. Dieses Auswahlverfahren fand online mit Hilfe standardisierter Fragebögen statt. Knapp ein Drittel der getesteten Personen schickte die Fragebögen nicht zurück, weitere Personen erfüllten nicht die notwendigen Kriterien für die Teilnahme. Ausgeschlossen wurden u. a. Personen, die noch nicht volljährig oder bereits in therapeutischer Behandlung waren; auch Dissoziationserfahrungen, psychotisches Erleben, schwere affektive Störungen sowie Alkohol- oder Drogenmissbrauch waren Ausschlusskriterien. So blieben $\mathrm{N}=96$ Personen, die an der Untersuchung teilnahmen. Von ihnen waren 89\% weiblich, 11\% männlich. Das Alter der Teilnehmer lag zwischen 18 und 68 Jahren, mit einem Durchschnitt von 35 Jahren. Sie alle hatten eine traumatische Erfahrung gemacht (Verlust einer nahe stehenden Person, sexueller Missbrauch, Inzest oder Vergewaltigung, Gewalterfahrungen) und litten unter den Symptomen einer PTB. Abbildung 9 gibt einen Überblick über das Auswahlverfahren und die Gründe für das Ausscheiden. Von den 96 teilnehmenden Personen wurden 49 mit der Interapy behandelt, die übrigen bildeten eine Wartekontrollgruppe. 


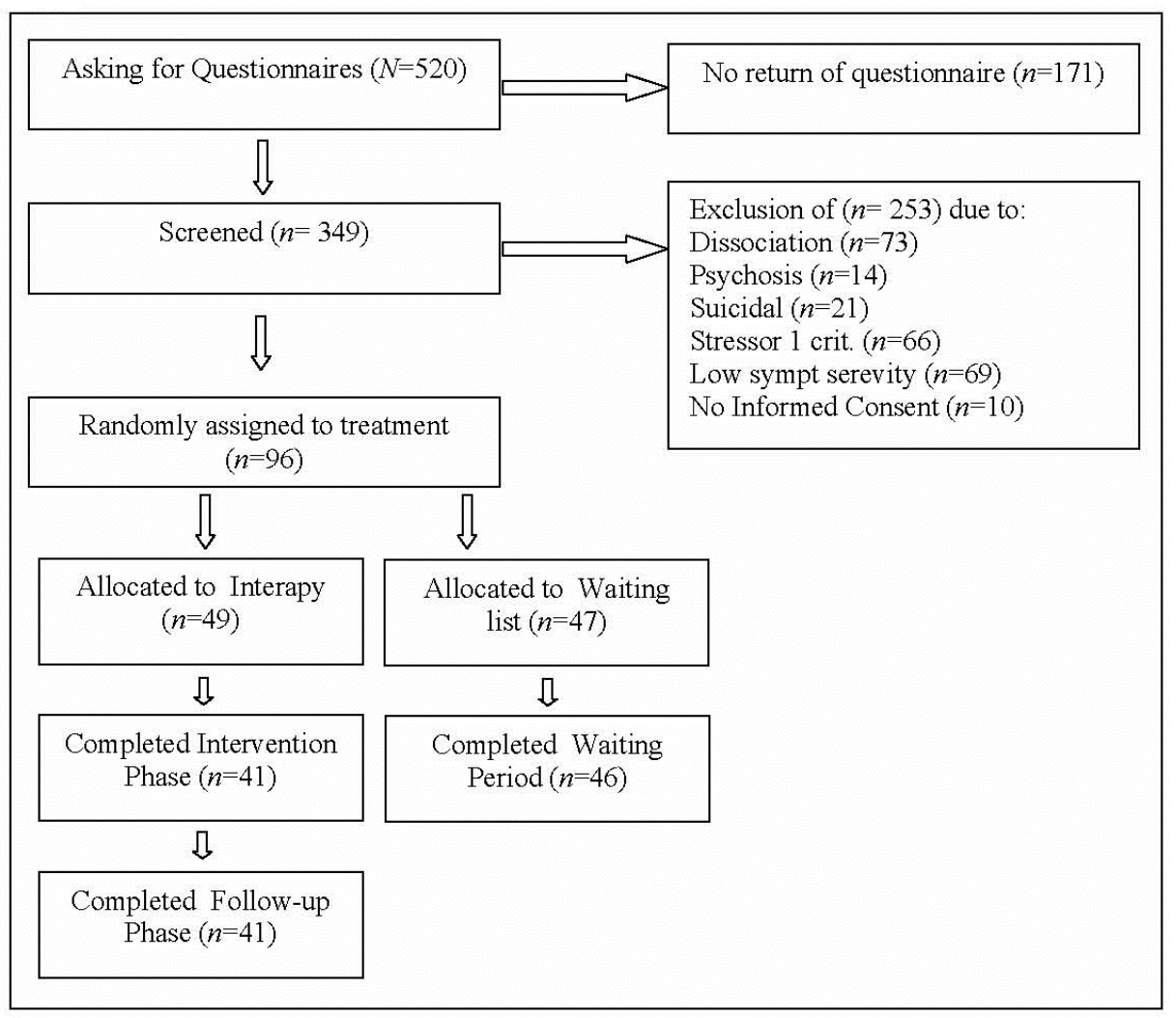

Abb. 9: Flussdiagramm zur Selektion der Untersuchungsteilnehmer (Knaevelsrud, 2005, S. 66).

Die Effekte wurden anhand folgender Instrumente ermittelt: Impact of Event Scale (rev.) zur Erfassung der PTB-Symptomatik (Weiss \& Marmar, 1997; dt. Übersetzung von: Maercker \& Schützwohl, 1998) sowie weiterer Fragebogen zur Erfassung von Ängstlichkeit, Depressivität und Somatisierungstendenzen. Die Werte wurden $\mathrm{zu}$ Beginn und nach Abschluss der Behandlung sowie in einer drei Monate und einer 18 Monate später erfolgten Follow-UpUntersuchung sowohl für die Experimental- als auch für die Wartekontrollgruppe erhoben. Mit einer MANOVA wurden die Ergebnisse ausgewertet und es zeigte sich, dass die Interaktion zwischen Gruppe und Prä-Post-Vergleich auf fast allen Dimensionen (mit Ausnahme einer Skala des Somatisierungsfragebogens) signifikant war. Das heißt also, dass die behandelten Personen eine signifikant höhere Symptomreduktion im fraglichen Zeitraum erzielten als die unbehandelte Wartekontrollgruppe. Somit kann die Behandlung mit der Interapy als erfolgreich angesehen werden. Aber wie lange halten die Effekte vor? Um dies herauszufinden wurden 3 Monate und 18 Monate nach Behandlungsende Nachuntersuchungen durchgeführt, in denen die PTB-Symptomatik erhoben wurde. Es konnte festgestellt werden, dass die Symptomausprägungen auch anderthalb Jahre nach Abschluss der Behandlung kaum über den Werten direkt nach dem Abschluss der Behandlung lagen (vgl. Abb. 10). Somit scheint die Interapy ein wirksames Verfahren zur Behandlung der PTB 
zu sein, mit Hilfe dessen sich auch langfristige Erfolge erzielen lassen. Dies ist insbesondere daher so beachtenswert, da das Verfahren selbst nur fünf Wochen dauert und in dieser Zeit nicht mehr als 10 Behandlungssitzungen (Schreibphasen) stattfinden. Somit handelt es sich um ein recht ökonomisches Verfahren.

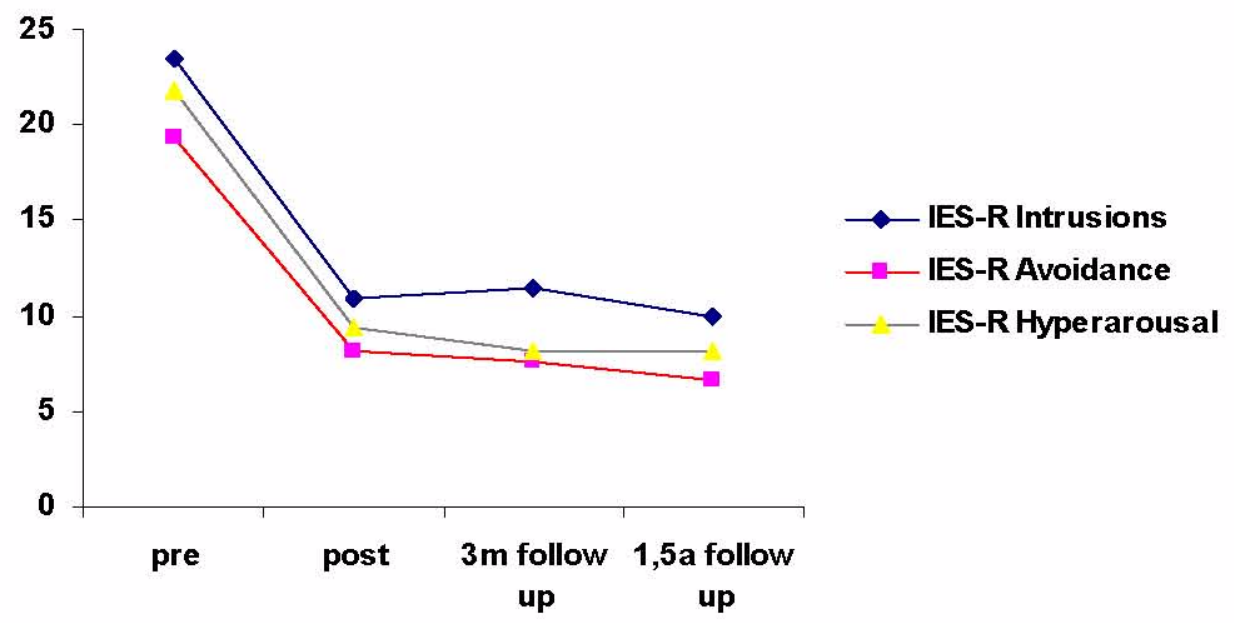

Abb. 10: PTB-Symptome vor Behandlungsbeginn (pre), nach dem Abschluss der Therapie (post), und in den beiden Nachuntersuchungen 3 Monate und 1,5 Jahre nach Behandlungsabschluss. Die Linien stehen für verschiedene Symptome, die mit dem IES-R erfasst wurden: Intrusionen, Vermeidungsverhalten (Avoidance) und Übererregbarkeit (Hyperarousal). (Maercker, 2005, S. 10).

Um auch letzte Zweifel an der Interapy auszuräumen, wurde auch die Zufriedenheit der therapierten Personen mit dem Verfahren erfasst. Auch hier waren die Ergebnisse sehr zufrieden stellend: $76 \%$ der Befragten $(\mathrm{N}=41)$ fanden den therapeutischen Kontakt angenehm und ganze $86 \%$ gaben an, auch per Internet eine persönliche Beziehung zum Therapeuten aufgebaut $\mathrm{zu}$ haben. Lediglich 12\% der Befragten gaben an, persönlichen Kontakt zum Therapeuten vermisst zu haben.

\subsubsection{Paartherapie nach dem Theratalk-Konzept}

Aufgrund des universitären Hintergrundes von Theratalk wurden bereits mehrere Untersuchungen sowohl $\mathrm{zu}$ den verwendeten Verfahren (Beer, 2004) als auch zur Wirksamkeit der Online-Therapie durchgeführt. Im Folgenden soll die von Beer und Breuer (2003, 2004) beschriebene Untersuchung zur Wirksamkeit des Paartherapie-Konzeptes Theratalk vorgestellt werden. Auch hier wird auf eine Beschreibung des Therapie-Vorgehens 
verzichtet, da diese sich bereits an anderer Stelle dieser Arbeit findet. Bei der von Beer \& Breuer (2003) beschriebenen Untersuchung handelt es sich um eine Wirksamkeitsstudie mit alternativ behandelter Kontrollgruppe. Bei den Studienteilnehmern handelte es sich um hilfesuchende Paare, in deren Partnerschaft kein Seitensprung in jüngster Vergangenheit vorlag oder bekannt geworden ist, von denen keiner ein Alkoholproblem hatte und mindestens ein Partner im Vortest (Dyadic Adjustment Scale von Spanier, 1976) den kritischen Wert von 100, der die Grenze zwischen „glücklich“ und „unglücklich“ markiert) unterschritten hatte. Die Teilnehmer der Experimentalgruppe unterzogen sich einer sechswöchigen Theratalk-Online-Therapie, die Kontrollgruppe nahm an einer verhaltenstherapeutischen Kurzzeit-Paartherapie mit Face-to-Face-Kontakt zwischen Therapeut und Patient teil. Die Zuweisung zu den Gruppen konnte hier nicht randomisiert erfolgen, weshalb in einem Vorabtest überprüft wurde, ob die Gruppen als äquivalent betrachtet werden können. Es zeigten sich bei den erhobenen Merkmalen (s. Tabelle 3) keine signifikanten Unterschiede.

Tab. 3: Eigenschaften der Untersuchungsteilnehmer, getrennt nach Online- und Face-to-face-Gruppe (erstellt nach den Angaben von Beer \& Breuer, 2003)

\begin{tabular}{l|l|l}
\multicolumn{2}{c|}{ Theratalk-Gruppe } & Face-to-Face-Gruppe \\
\hline Fallzahl & 22 Paare & 20 Paare \\
\hline Durchschnittsalter & 39 Jahre & 41 Jahre \\
\hline durchschn. Beziehungsdauer & 11,4 Jahre & 14,4 Jahre \\
\hline durchschn. Kinderzahl & 1,2 Kinder & 1,3 Kinder \\
\hline Familienstand & $59 \%$ verheiratet & $65 \%$ verheiratet \\
\hline
\end{tabular}

Beide Gruppen machten zu Beginn der jeweiligen Behandlung mit ihrem Therapeuten das Oral History Interview (Buehlmann, Gottmann, Katz, 1992). Insgesamt hatten die Paare in der Face-to-Face-Therapie vier Kontakte mit ihrem Therapeuten, die jeweils zwei Stunden dauerten, die Paare in der Theratalk-Gruppe hatten 5 kurze E-MailKontakte pro Woche, über einen Zeitraum von sechs Wochen. Beide Therapie-Formen zielten explizit auf die Verringerung der Ziel-Schwierigkeiten ab, z.B. durch das Setzen von Teilzielen. In einer Nachuntersuchung 3 Monate nach dem Ende der Therapie wurden wieder die DAS-Werte ermittelt. Die Autoren beschreiben die Effekte für die beiden Gruppen und nach Geschlecht getrennt (vgl. Abb. 11): Nach der 
Face-to-Face-Therapie hatten $52 \%$ der Frauen und $59 \%$ der Männer, die im Prätest einen DAS-Wert < 100 hatten, einen DAS-Wert von 100 oder größer und waren somit im Lauf der Therapie „glücklich“ geworden. Die Werte für die TheratalkGruppe fielen etwas besser aus, hier hatten $55 \%$ der Frauen und $63 \%$ der Männer ihren Wert auf mindestens 100 erhöht. Somit konnten beide Therapie-Formen bei beiden Geschlechtern eine signifikant größere Zufriedenheit mit ihrer Beziehung erreichen.
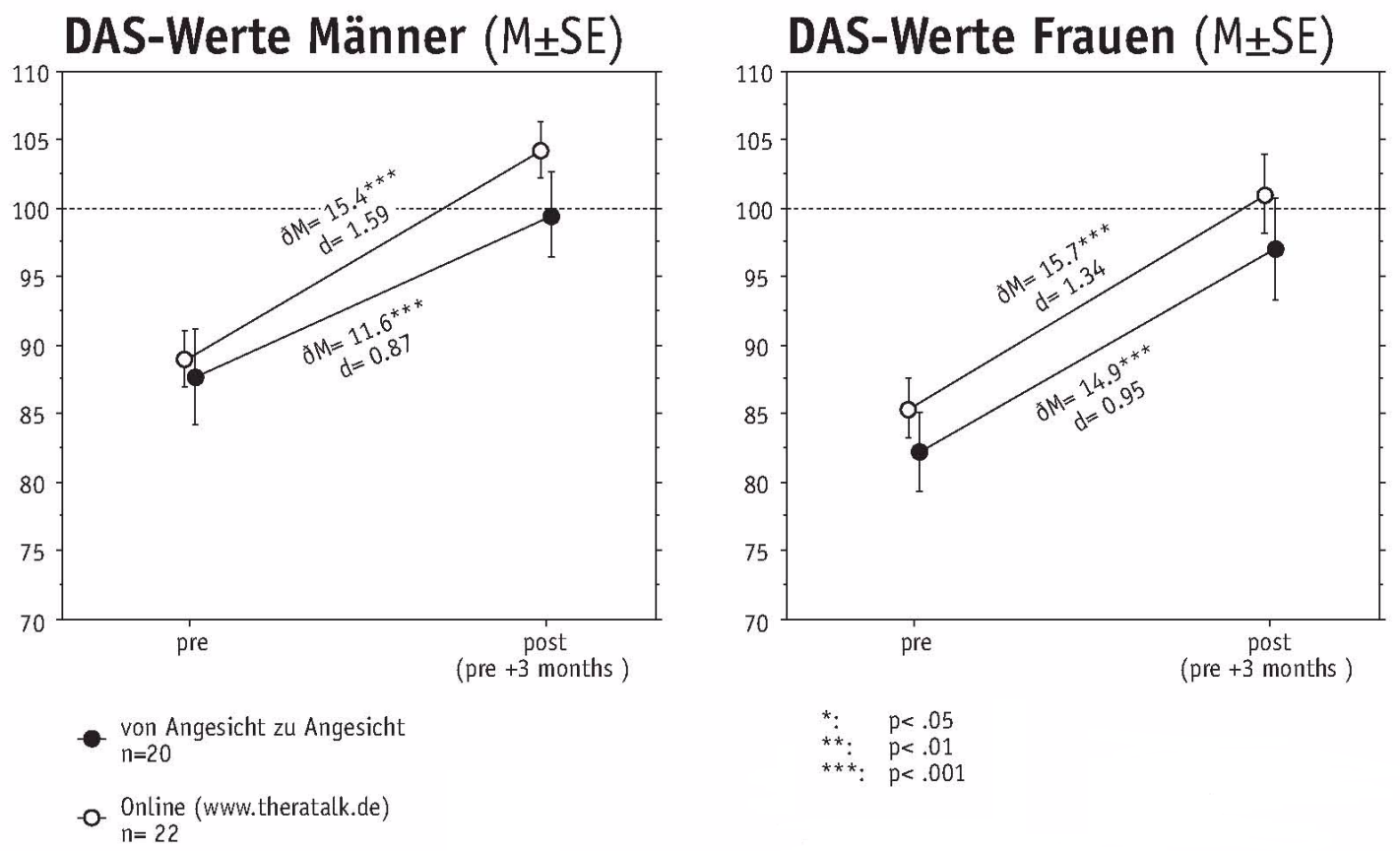

Abb. 11: DAS-Werte vor und nach der Therapie getrennt nach Geschlechtern (Beer \& Breuer, 2003, S. 8)

Leider versäumen die Autoren jedoch, die Effekte der beiden Gruppen miteinander zu vergleichen und geben nicht an, wie die Theratalk-Behandlung im Vergleich mit der herkömmlichen Therapie abschneidet. Wahrscheinlich jedoch sind die Unterschiede nicht signifikant.

Auch Untersuchungen zur Ökonomie der neuen Behandlungsform fehlen leider. Die auf der Internetseite und in der Presse als besonders effiziente Behandlungsform angepriesene Theratalk-Behandlung erfordert wesentlich mehr Kontakte zwischen Patient und Therapeut als die herkömmliche Therapie und es ist daher fraglich, ob die Internet-Intervention der herkömmlichen Therapie in diesem Punkt überlegen ist. 
Nichtsdestotrotz sind die Ergebnisse sehr erfreulich, da sie die Wirksamkeit der OnlineTherapie bestätigen und so eine Alternative für Paare bietet, die den Gang zum Therapeuten scheuen.

\subsubsection{Internetchatgruppen zur Nachbehandlung}

Die Wirksamkeit und Akzeptanz von Internetchats in der Nachbehandlung psychischer und psychosomatischer Störungen wurde von Kordy und Kollegen (Kordy, Golkaramnay, Wolf, Haug \& Bauer, 2006) in Kooperation mit der Techniker Krankenkasse durchgeführt. Dabei wurden Patienten, die zwischen November 2001 und März 2003 in der Panorama-Fachklinik für Psychosomatik, Psychotherapeutische Medizin und Naturheilverfahren in Scheidegg/Allgäu in Behandlung und bei der entsprechenden Krankenkasse versichert waren, die Teilnahme an der Untersuchung angeboten, von denen 228 in die Erhebung eingingen. Die Hälfte dieser Patienten erhielt die Möglichkeit, im Anschluss an die Behandlung 12-15 Wochen an einer Internetchatgruppe teilzunehmen, die andere Hälfte stellte die Kontrollgruppe dar und erhielt keine Nachbehandlung, sondern füllte lediglich die Fragebögen zur Qualitätssicherung 6 Monate nach Abschluss der stationären Behandlung aus. Die Diagnoseverteilung unter den teilnehmenden Patienten spiegelt die Verteilung der Klinikpatienten wider; am häufigsten waren affektive Störungen, Persönlichkeitsstörungen, somatoforme Störungen sowie Verhaltensauffälligkeiten.

An der Studie teilnehmen konnten alle Patienten, die im fraglichen Zeitraum in der PanoramaKlinik in stationärer Behandlung und bei der TK versichert waren; einziges Ausschlusskriterium waren psychotische Störungen. Die Zuweisung zu den Gruppen erfolgte nicht randomisiert, da nicht alle Patienten in der Lage oder bereit waren, an den Chatgruppen teilzunehmen. Um Zufallseinflüsse minimieren zu können, wurden matched pairs gebildet, wobei neben den demographischen Merkmalen und der Internet-Erfahrung auch die psychische Beeinträchtigung berücksichtigt wurde. Tabelle 4 zeigt die soziodemographischen Merkmale der Studienteilnehmer getrennt nach den beiden Gruppen. Die Untersuchungsteilnehmer unterschieden sich von den durchschnittlichen Patienten der Panorama-Klinik lediglich durch ihr etwas niedrigeres Alter und den - wahrscheinlich dadurch bedingt - höheren Anteil Lediger.

Tab. 4: Demographische Merkmale der Studienteilnehmer getrennt nach Chat- und Kontrollgruppe (nach Angaben von Kordy et al., 2006) 


\begin{tabular}{l|l|l} 
& Chatgruppe & Kontrollgruppe \\
\hline Fallzahl & 114 & 114 \\
\hline Durchschnittsalter & 41,7 Jahre $(\mathrm{SD}=11,6 \mathrm{~J})$ & $43,2 \mathrm{Jahre}(\mathrm{SD}=12,9 \mathrm{~J})$ \\
\hline Geschlechterverhältnis & $78,9 \%$ $\%$ & $72,8 \% \%$ \\
\hline Familienstand & $53,3 \%$ verheiratet & $49,1 \%$ verheiratet \\
\hline
\end{tabular}

Die Wirksamkeit der Teilnahme an der Internet-Chatgruppe wurde mit dem Auffälligkeitskriterium des Stuttgart-Heidelberger Modells der Qualitätssicherung (Kordy, Hannöver \& Bauer, 2003) erfasst; als Nebenkriterien wurden psychische Gesundheit, körperliches Wohlbefinden, direkte Veränderungsmessungen und die Patientenzufriedenheit erfasst. Die Beurteilung der Akzeptanz erfolgte anhand mehrerer Indikatoren: Bereitschaft zur Teilnahme, Anwesenheitsrate bei den Chatterminen, Abbrecherquote und selbst eingeschätzte Zufriedenheit, die im Anschluss an die einzelnen Sitzungen und nach Abschluss der Behandlung erfasst wurde.

Die Akzeptanz der Internetbrücke zur Nachbehandlung kann als sehr groß bewertet werden: Bereits vor deren Installation war sie einem Großteil der Patienten bekannt und die meisten Befragten (71,2\%) äußerten Interesse an der Teilnahme. Auch von den Patienten, die schließlich an der Studie teilnahmen, wurde die Gruppe offensichtlich gut aufgenommen: die Anwesenheitsrate betrug stets mindestens $85 \%$, lediglich $9,7 \%$ brachen die Behandlung vorzeitig ab. Die genannten Gründe für den Abbruch sprechen dafür, dass der vorzeitige Ausstieg in den wenigsten Fällen auf eine Unzufriedenheit mit der Chatgruppe zurückzuführen ist.

Folglich waren die Bewertungen der Chatgruppe im Anschluss an die Behandlung sehr gut: 90\% der Teilnehmer waren mit der Gruppe zufrieden, $83 \%$ bewerteten sie als sinnvolle Ergänzung zur herkömmlichen Behandlung. Die Bewertung der einzelnen Sitzungen gleicht denen der Gesamtbehandlung: auch hier äußerten die Teilnehmer ihre Zufriedenheit und gaben an, sich akzeptiert zu fühlen, sich frei aussprechen zu können und schätzten die Gruppe als hilfreich ein. Nur ein kleiner Teil der Teilnehmer fühlte sich durch die Technik eingeschränkt. Die Frage der Akzeptanz einer Internetbrücke kann also positiv beantwortet werden, aber wie steht es um deren Wirksamkeit?

Die Beurteilung der Effektivität erfolgte anhand von Fragebögen, die die Teilnehmer beider Gruppen sechs Monate nach dem Ende ihrer stationären Behandlung ausfüllen mussten und die mit Hilfe eines Chi-Quadrat-Tests und t-Tests für abhängige Gruppen mit einer a prioriIrrtumswahrscheinlichkeit von $\alpha=0.05$ ausgewertet wurden. 
Es zeigte sich, dass der Verlauf des Zielkriteriums „Auffälligkeit“ bei denjenigen Patienten, die an der Chatgruppe teilnahmen, wesentlich positiver war als bei der Vergleichsgruppe: während der Prozentsatz auffälliger Patienten bei der Kontrollgruppe im Laufe von sechs Monaten von $12 \%$ auf 33,7\% anstieg, stieg er unter den Chatteilnehmern trotz des etwas höheren Ausgangswertes von 13,8 \% lediglich auf 21,8 \% an. 64,3\% der Chatteilnehmer konnten ihren psychischen Zustand nach der Entlassung sogar verbessern, in der Kontrollgruppe gelang dies lediglich $36,4 \%$ der Fälle.

Auch die Ergebnisse für die Nebenzielkriterien fallen positiv aus. Die körperliche Befindlichkeit (erfasst mit dem GBB; Brähler \& Scheer, 1995) der Patienten beider Gruppen verbesserte sich im Lauf der stationären Behandlung und stabilisierte sich im Anschluss an diese - trotz geringer anfänglicher Verschlechterung - im Normalbereich. Die Unterschiede zwischen den Gruppen waren in diesem Fall nicht signifikant.

Das psychische Befinden (erfasst mit der Symptom-Checkliste SCL-90-R von Franke, 1995 und dem Ergebnisfragebogen EB-45 von Lambert, Hannöver, Nisslmüller, Richard \& Kordy, 2000) der beiden Gruppen war zu Beginn der Behandlung im Mittel fast identisch, in der Katamnese zeigten sich jedoch deutliche Unterschiede zugunsten der Chatteilnehmer. Die Ergebnisse beider Instrumente sind für die Chatteilnehmer positiver als für die unbehandelte Vergleichsgruppe. Beim SCL-90-R ist der Mittelwertsunterschied zwischen den Gruppen mit $\mathrm{p}=0.13$ zwar nicht signifikant, beim EB-45 ist das Ergebnis deutlicher: der Mittelwertsunterschied von 12,45 ist hochsignifikant $(\mathrm{p}=0.000)$.

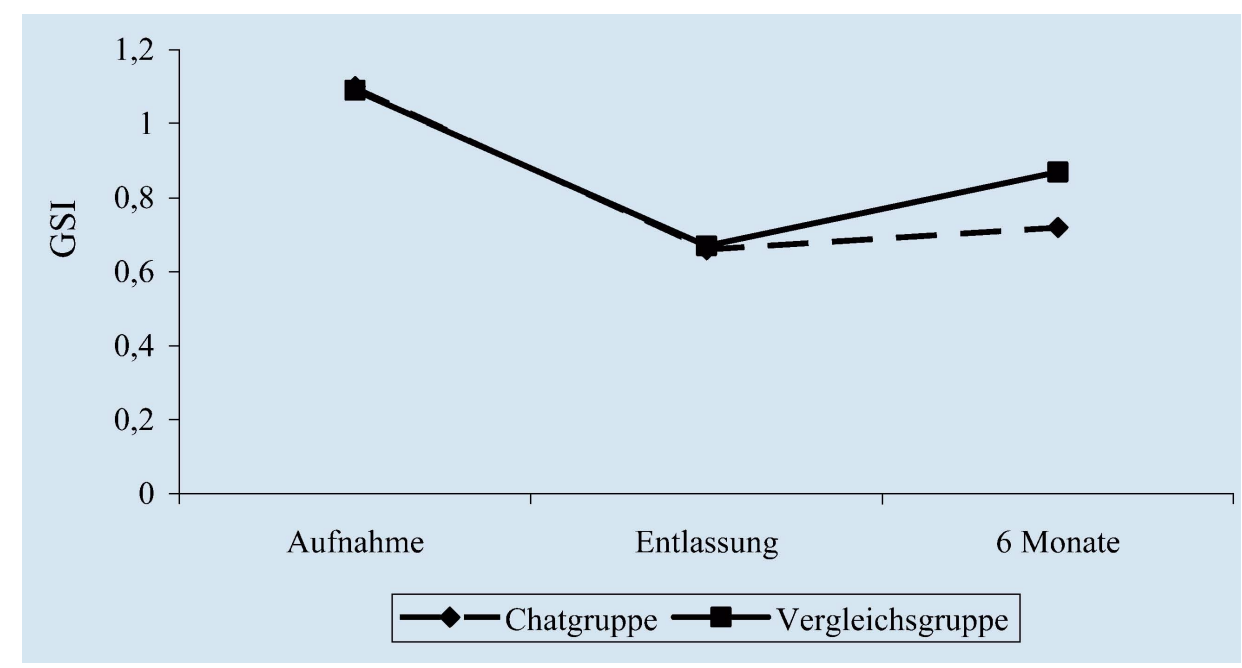

Abb. 12: Psychische Beeinträchtigung (SCL-90-R GSI) der Chat- und Kontrollgruppe (Kordy et al., 2006, S. 151) 
Diesen Ergebnissen entsprechend schätzen die Teilnehmer der Chatgruppe ihren psychischen und physischen Zustand etwas positiver ein als die der Kontrollgruppe.

Zusammenfassend lässt sich feststellen, dass die Einrichtung einer Chatgruppe unter den Patienten auf sehr große Akzeptanz stößt und sich gleichzeitig als hilfreich für die Nachbehandlung erweist. „Eine Internetbrücke zwischen Fachklinik und Alltag eröffnet neue Möglichkeiten $\mathrm{zu}$ einer besseren Integration stationärer und ambulanter Versorgung und damit letztlich zu einer Optimierung der Versorgung gerade für Patienten, für die eine therapeutische Betreuung über längere Zeit notwendig ist“" (Kordy et al., 2006). Die vorliegende Untersuchung zeigt deutlich, dass die Nachversorgung auch via Internet zu weiteren Verbesserungen des Befindens bzw. zu einer größeren Stabilität desselben führt. Zu klären bleibt jedoch die Frage, wie die Effekte einer Internet-Nachsorge im Vergleich zu einer ambulanten Nachbehandlung abschneiden und ob diese Form der Nachsorge für alle Patienten gleichermaßen geeignet ist, da in dieser Untersuchung die Ergebnisse leider nicht nach der Ausgangsdiagnose der Patienten aufgeschlüsselt wurden.

\subsubsection{Nachbehandlung bei Bulimia nervosa mit Hilfe von Textnachrichten}

Die Untersuchung von Robinson und Kollegen (Robinson, Perkins, Bauer, Hammond, Treasure \& Schmidt, 2006) beschäftigt sich ebenso wie die Studie von Kordy et al. (2006) mit einer Kombination aus herkömmlicher Therapie und digitalisierter Nachbehandlung. Die wesentlichen Unterschiede sind darin zu sehen, dass es bei Robinson et al. um die Nachsorge im Fall einer spezifischen Störung, der Bulimia nervosa, geht und die Patienten hier über Textnachrichten (SMS) Kontakt aufnehmen.

An der Untersuchung nahmen 21 Patienten teil, die sich zuvor einer stationären Therapie unterzogen hatten. Bei allen war eine Bulimia nervosa oder eine nicht näher bestimmte Essstörung nach DSM-IV diagnostiziert worden. Die Teilnehmer waren überwiegend weiblich (95,3 \%), zwischen 19 - 48 Jahren alt (Median: 26 Jahre). Der BMI lag im Median bei $17,8 \mathrm{~kg} / \mathrm{m}^{2}$. Die Patienten litten seit weniger als zwei Jahren bis zu mehr als 20 Jahre unter der genannten Störung. Alle Patienten nahmen im Anschluss an die stationäre Behandlung an dem Nachbehandlungsprogramm mit Textnachrichten teil; eine Kontrollgruppe gab es nicht.

In einem Prätest wurden die Symptome zu Beginn des SMS-Programms erfasst. Das Programm war auf sechs Monate angelegt, innerhalb derer die Patienten einmal wöchentlich eine Textnachricht in standardisiertem Format senden sollten, in denen sie fünf Fragen zu ihrer Symptomatik und ihrer psychischen Verfassung beantworten sollten. Diese Nachrichten 
wurden von einem internetbasierten semi-automatisierten Programm erfasst und mit einer Nachricht aus einem zuvor programmierten Nachrichtenpool beantwortet. Darüber hinaus wurden die Antworten der Patienten auf die fünf Fragen gesammelt und gingen in ein monatliches Feedback zum Verlauf der Symptomatik ein.

Das Programm, mit Hilfe dessen die Nachrichten ausgewertet und beantwortet wurden, ist an der Universität Heidelberg entwickelt worden. Eine Pilotstudie hat die Akzeptanz dieses Verfahrens bei deutschen Patienten bestätigt.

$\mathrm{Zu}$ Beginn des Programms wurden die Teilnehmer aufgefordert, ihre Motivation zur Teilnahme auf einer fünfstufigen Likert-Skala $(0=$ nicht motiviert, $4=$ hoch motiviert $)$ einzuschätzen, wobei sich ein Median von 3 ergab. Trotz dieser überdurchschnittlich hohen Ausgangsmotivation, brachen 12 der anfänglich 21 Patienten (57.14 \%) das Programm vorzeitig ab. Auch die neun Patienten, die über die gesamte Dauer von sechs Monaten im Programm blieben, nahmen nicht - wie gefordert - einmal wöchentlich Kontakt auf. Die Anzahl gesendeter Nachrichten betrug im Median 13 (zwischen 3 und 23), die geforderte Anzahl betrug 26. Diese Zahlen deuten bereits darauf hin, dass die Akzeptanz dieses Programms nicht sehr gut war. In einem Posttest, der allen 21 ursprünglichen Patienten zugeschickt wurde, sollte die Meinung, die die Teilnehmer über das Programm hatten, erfasst werden. Hier bestätigte sich die Vermutung: Auch wenn die Patienten der Aussage zustimmten, dass der mit der Teilnahme einhergehende Aufwand gering war, gaben dennoch $85,71 \%$ der Teilnehmer an, dass sie wahrscheinlich oder ganz bestimmt nicht noch einmal an dem Programm teilnehmen würden und 86,67 \% sagten, sie würden es nicht weiterempfehlen. Mehr als die Hälfte der Teilnehmer (53,3 \%) bemängelten den fehlenden persönlichen Kontakt.

Die Wirksamkeit des Programms kann nicht abschließend beurteilt werden. Die Autoren nennen hierzu folgende Zahlen: Zu Beginn des Programms waren 9,5\% der Patienten abstinent und 38,10 \% subklinisch, am Ende der Nachbehandlung 29,4 \% abstinent und 17,7 $\%$ subklinisch. Auf den ersten Blick könnte dies als Erfolg gewertet werden, es muss aber darauf hingewiesen werden, dass der Anteil der Patienten mit klinisch relevanter Symptomausprägung relativ stabil blieb, und - wichtiger noch - den Zahlen unterschiedliche Stichproben zugrunde liegen. Während im Prätest alle 21 Personen erfasst wurden, liegen den Abschlussdaten rücklaufbedingt nur 17 Fälle zugrunde.

Auch wenn die Autoren auf die positiven Ergebnisse der Heidelberger Studie (Bauer, Percevic, Okon, Meermann \& Kordy, 2003) hinweisen, so dürfen die Ergebnisse dieser Untersuchung doch nicht auf die leichte Schulter genommen werden. Es liegt nahe, dass hier 
ein Zuviel an Technik eingesetzt wurde und der zwischenmenschliche Kontakt deutlich zu kurz kam und es somit zu einer einseitigen Kommunikation kam. Zukünftige Forschung sollte sich daher mit der Frage beschäftigen, welcher Grad der Technisierung in der psychologischen Intervention tatsächlich sinnvoll ist und was das Minimum an zwischenmenschlichem Kontakt - wenn auch computermediiert - ist. Darüber hinaus sind vergleichende Untersuchungen des Programms, auch nach einer eventuellen Revision, mit unbehandelten und alternativ behandelten Kontrollgruppen dringend geboten.

\section{Diskussion}

\section{Möglichkeiten und Grenzen von Beratung und Online-Therapie}

In der vorliegenden Arbeit sollte ein Überblick über die möglichen Angebote zur internetbasierten Selbsthilfe, Beratung und Therapie gegeben werden und der aktuelle Stand der Forschung zu diesem Thema aufgezeigt werden. Abschließend sollen nun Möglichkeiten und Grenzen der internetbasierten Interventionsangebote ausgeleuchtet werden.

Die Vorteile internetbasierter Beratungs- und Therapiekonzepte sind nach den vorliegenden Ergebnissen nicht von der Hand zu weisen: Zum einen sind solche Angebote für eine Vielzahl von Personen unabhängig vom Wohnort zugänglich und können so in Fällen unzureichender psychosozialer Infrastruktur eine willkommene Ergänzung zum vorhandenen Angebot darstellen. So kann internetbasierte psychotherapeutische Intervention dazu beitragen, dass auch solche Menschen Hilfe in Anspruch nehmen können, denen es ansonsten auf Grund ihrer Wohnortlage oder körperlicher respektive psychischer Einschränkungen nicht möglich wäre, eine Beratungs- oder Therapieeinrichtung vor Ort aufzusuchen.

Neben den professionellen Angeboten für Beratung und Therapie dürfen aber die Möglichkeiten zur Selbsthilfe über das Internet nicht aus dem Blick geraten. Eichenberg und Laszig weisen darauf hin, dass ,die Partizipation an virtuellen Selbsthilfegruppen (...) neue Kommunikationserfahrungen und die Publikation einer eigenen Homepage eine Ausdrucksmöglichkeit (bietet), um auf die eigenen Probleme und Anliegen aufmerksam zu machen“ (Eichenberg \& Laszig, 2003, S. 187). Insofern können gerade solche Patienten, denen es an Möglichkeiten zum sozialen Austausch vor Ort fehlt, von OnlineSelbsthilfegruppen profitieren.

Internetberatung und -Therapie können auch für solche Menschen, die einen Gang zum Therapeuten scheuen, hilfreich sein. Da die Kontaktaufnahme hier anonym erfolgt und für Dritte verborgen bleibt, sind die Hemmschwellen bei der Inanspruchnahme von 
internetbasierten Angeboten wesentlich geringer als bei herkömmlichen Einrichtungen vor Ort.

Neben der Wohnortunabhängigkeit und Anonymität der Kontaktaufnahme bieten Internetangebote auch den Vorteil der Zeitunabhängigkeit. Die Kontaktaufnahme kann zu jeder Tages- oder Nachtzeit erfolgen, wann immer der Betroffene das Bedürfnis danach verspürt. Professionelle Angebote sichern zudem eine schnelle Beantwortung der Anfragen $\mathrm{zu}$, so dass Wartezeiten, die bei niedergelassenen Beratern oder Therapeuten an der Tagesordnung sind, vermieden werden können.

Darüber hinaus können die ausgetauschten Nachrichten archiviert werden, was nicht nur für den Betroffenen, sondern auch für den Behandelnden von Vorteil ist. Auf diese Weise können Dialoge auch nach längerer Zeit noch ins Gedächtnis zurück gerufen und der Behandlungsverlauf kann nachvollzogen werden. So kann sich der Betroffene Fortschritte vor Augen halten und Ratschläge des Therapeuten immer wieder ins Gedächtnis rufen. Auf der anderen Seite wird die Kommunikation so transparent und eine Supervision erleichtert, da objektiviert: es muss nicht mehr auf subjektiv gefärbte Erinnerungen des Therapeuten zurückgegriffen werden, wenn der gesamte Behandlungsverlauf in schriftlicher Form vorliegt. Aber nicht nur als alleiniges Mittel der Intervention, auch zur Ergänzung herkömmlicher Angebote können internetunterstützte Maßnahmen von Nutzen sein. Eine Anamnese über das Internet - oder Mobilmedien - kann die Selektion von Patienten für ein spezielles Therapieangebot erleichtern und dabei Zeit und Verwaltungsaufwand sparen. Aber auch der Abschluss einer Behandlung und die Wiedereingliederung in den Alltag nach einem Klinikaufenthalt können durch eine Internet (oder SMS-)Brücke erleichtert werden, wie Kordy und Kollegen zeigen konnten.

Die genannten Vorzüge internetbasierter Interventionsangebote dürfen nicht darüber hinwegtäuschen, dass diese auch eine Kehrseite haben. Gerade im Bereich der Selbsthilfe und der (kommerziellen) Beratung besteht die Gefahr, auf nicht-professionelle Gesprächspartner zu treffen und falsche Informationen und Ratschläge zu erhalten, die nicht zur Verbesserung sondern - im schlimmsten Fall - sogar zu einer Verschlechterung der Symptomatik führen können. Aus diesem Grund ist eine dringende Aufgabe von Wissenschaftlern und Berufsverbänden in der Schaffung von Qualitätssicherungsmaßnahmen und Kontrollgremien zu sehen, um den Hilfesuchenden fundierte und klare Informationen über die Professionalität und Qualität bieten zu können.

Es muss auch darauf hingewiesen werden, dass internetbasierte Therapieangebote - nicht zur Behandlung aller Störungen in gleichem Maße geeignet sind (vgl. Ott, 2003). In manchen 
Fällen ist diese Form der Intervention sogar kontraindiziert. Nämlich dann, wenn das Therapieziel darin besteht, das Ausmaß der Internetnutzung $\mathrm{zu}$ reduzieren oder dem Betroffenen die Anbahnung sozialer Kontakte zu erleichtern. In solchen Fällen von pathologischer Internetnutzung oder sozialem Rückzug, sollten internetbasierte Verfahren allenfalls zur Anbahnung der Therapie eingesetzt werden. Manche Autoren vertreten die Ansicht, dies auch bei anderen Problembereichen der Fall ist und Online-Intervention nie allein stehen sollte.

„Beim derzeitigen Stand der Diskussion kann davon ausgegangen werden, dass Online-Beratung eine wichtige Funktion bei der Informationsvermittlung einnimmt und zur Anbahnung einer Psychotherapie geeignet erscheint. OnlineBeratung ist jedoch von der Online-Therapie abzugrenzen - beide Verfahren können die klassische Psychotherapie nicht ersetzen.“

(Laszig \& Rieg, 2004, S. 115)

Den Einsatzmöglichkeiten internetbasierter Intervention sind also nicht nur durch das Störungsbild des Patienten, sondern auch durch die Bereitschaft und Einstellung des Therapeuten Grenzen gesetzt. Die theoretische Ausrichtung des Therapieansatzes kann der Verwendung von computergestützen Verfahren entgegenstehen: nicht ohne Grund gibt es bis zum heutigen Zeitpunkt kaum Konzepte aus dem Bereich klientenzentrierter und der psychoanalytischen oder tiefenpsychologischen Psychotherapie. Der Stellenwert, der dem zwischenmenschlichen Kontakt in diesem Ansatz eingeräumt wird, scheint andere Kommunikationsformen als das Face-to-Face Gespräch auszuschließen. Auch der Hier-undJetzt-Ansatz von Yalom betont die Notwendigkeit persönlicher Interaktion und spricht so gegen den Einsatz internetbasierter Maßnahmen. Grundannahme dieses Ansatzes ist es, dass „die große Mehrzahl derjenigen, die eine Therapie beginnen, Probleme in Beziehungen (hat): im Großen und Ganzen verzweifeln Leute deswegen am Leben, weil sie unfähig sind, dauerhafte und befriedigende zwischenmenschliche Beziehungen einzugehen und aufrechtzuerhalten. (...)

Die zweite Annahme - dass die Therapie ein sozialer Mikrokosmos ist - verweist auf die Tatsache, dass sich irgendwann (...) die zwischenmenschlichen Probleme des Patienten im Hier und Jetzt der therapeutischen Beziehung manifestieren werden. Wenn der Patient oder die Patientin sich im Alltagsleben fordernd oder ängstlich oder arrogant oder distanziert oder verführerisch oder dominant oder rechthaberisch oder sonst wie zwischenmenschlich schlecht angepasst verhält, so 
werden sich diese Wesenszüge in die Beziehung des Patienten zum Therapeuten einschleichen.“

(Yalom, 2002, S. 62f)

Die Therapie nach tiefenpsychologisch/psychoanalytischem Ansatz setzt also voraus, dass der Therapeut die ungünstigen Verhaltensweisen des Patienten, die dieser im sozialen Austausch an den Tag legt und die auch im therapeutischen Kontakt offensichtlich werden, erkennt und dem Betroffenen Hilfestellung bei der Überwindung derselben gibt. Dies ist nur im persönlichen Kontakt möglich, da die Pseudonymität des Internets und zeitversetzte Kommunikation es dem Patienten ermöglichen, seine gewohnten Verhaltensweisen zu verbergen. So besteht die Gefahr, dass dem Therapeuten wesentliche Informationen zur Behandlung fehlen.

Tatsächlich ist die Online-Intervention noch mehr auf die Compliance und Offenheit des Patienten angewiesen als herkömmliche Beratung und Therapie. Deutlich wird dies auch am Beispiel der Essstörungen. Der anonyme Kontakt via Internet ermöglicht dem Patienten die Verschleierung von Gesundheitszustandes und Gewichtstatus. Diese Tatsache muss auch bei der Bewertung der von Robinson et al. (2006) sowie von Bauer et al. (2006) berichteten Ergebnisse bei der Nachbehandlung von Bulimie-Patienten berücksichtigt werden.

Letztlich bleibt festzustellen, dass - ungeachtet aller positiven Befunde und neuer Entwicklungen - die herkömmlichen Behandlungsformen nie gänzlich durch internetbasierte Interventionsformen ersetzt werden können. Da Online-Beratung und -Therapie immer durch den Patienten initiiert werden, greifen diese nicht bei dem ganzen Spektrum psychischer Störungen. Die Kontaktaufnahme des Patienten setzt nämlich Leidensdruck voraus. Störungen, bei denen Krankheitseinsicht (z.B. Schizophrenie) und Leidensdruck (z. B. Anorexie) fehlen, sind durchaus behandlungsbedürftig, können aber nicht durch OnlineAngebote abgedeckt werden. Das gleiche gilt auch für Fälle, in denen Fremdgefährdung (z. B. conduct disorder, antisoziale Persönlichkeitsstörung) besteht.

Auch für Patienten, die sich schriftlich nicht ausdrücken können (z. B. Kleinkinder, Personen mit Intelligenzminderung) werden weiterhin auf herkömmliche Behandlungsangebote angewiesen sein. Eine vollständige Verlagerung psychotherapeutischer Intervention in den Cyberspace ist also nicht zu erwarten. Diese Ansicht vertritt auch Sonnenmoser, wenn sie darauf hinweist, dass „,die Internet-Psychotherapie nur für einen bestimmten Patientenkreis und für experimentierfreudige Psychotherapeuten infrage (kommt)“ (Sonnenmoser, 2005, S. 166). 
Nichtsdestotrotz werden internetbasierte Interventionen auch in der Zukunft weiterhin an Bedeutung für die Praxis der Psychotherapie und Beratung gewinnen und sollten daher auch weiterhin Gegenstand der Forschung sein. Offene Fragen, die bislang noch nicht zufriedenstellend beantwortet werden konnten, betreffen nicht nur die Qualitätssicherung im Internet. Wenig beachtet wurde bisher auch die Sicht des Therapeuten. „Wie Psychotherapeuten den E-Mail-basierten Kontakt zu ihren Klienten empfinden, wurde bisher noch nicht untersucht“, konstatiert auch Sonnenmoser (2005, S. 166). Zukünftige Forschung sollte daher der Frage nachgehen, inwiefern die therapeutische Beziehung durch den Einsatz computervermittelter Kommunikation verändert wird und welche Bedingungen gegeben sein müssen, dass die Allianz auch ohne physische Präsenz gelingen kann.

Wie die Befunde von Robinson et al. (2006) gezeigt haben, wird übermäßige Standardisierung und Digitalisierung der Kommunikation von den Patienten nicht wohlwollend aufgenommen und führt zu hohen Abbruchquoten. Es sollte daher dringend die Frage geklärt werden, welches $\mathrm{Maß}$ an Technisierung akzeptable und welches Minimum an zwischenmenschlichem Kontakt (wenn auch computervermittelt) von Nöten ist. Keinesfalls darf die Patientenzufriedenheit zugunsten der Medialisierung und Ökonomisierung des Kontakts vernachlässigt werden. 


\section{Zusammenfassung:}

In der vorliegenden Arbeit wurden Hintergründe und Möglichkeiten internetbasierter Therapie- und Beratungsmöglichkeiten aufgezeigt. Darüber hinaus wurde ein vom Autor selbst durchgeführtes Projekt internetbezogener Beratung vorgestellt.

Neben der Geschichte des Internets bzw. der computervermittelten Kommunikation wurden insbesondere verschiedene, vor der „Internet-Ära“ entwickelte, Kommunikationstheorien vorgestellt und mit dem heutigen Status Quo verglichen.

Ferner wurde die Rolle des Internets in der Psychotherapie im Besonderen beleuchtet, sowie die sich hieraus ergebenen Qualitätsstandards einer solchen computervermittelten Kommunikation.

Abschließend wurden empirische Befunde zur Online-Therapie zusammengetragen und anhand einiger ausgewählter Studien vorgestellt.

Hierbei zeigten sich zum Teil deutliche Vorteile in der Nutzung dieser relativ jungen Form von Kommunikation mit Patienten. So eignet sich diese Form des Kontaktes besonders unter dem Anspruch der Gewährleistung von Anonymität und Wohnortsunabhängigkeit, sowie in der poststationären Nachbetreuung bereits anbehandelter Patienten.

Auch zeigten sich hierbei eindeutige Grenzen bei einer derartigen Form der Kommunikation. Nicht jede Störung, wie z.B. Sucht oder Störungen des Sozialverhaltens, kann und sollte auf diese Weise behandelt werden. Auch schützt die Anonymisierung die das Internet bietet, den Patienten nicht vor unqualifizierten Beratungsangeboten.

Sicherlich jedoch wird auch die Online-Therapie in Zukunft eine große Rolle spielen. 


\section{Literaturverzeichnis}

Baran, P. (1964). On distributed communications. Santa Monica, Cal.: Rand Corporation. Verfügbar unter: www.rand.org/pubs/researchmemoranda/2006/ RM3420.pdf [25.04.2007]

Bauer, S., Percevic, R., Okon, C., Meermann, R. \& Kordy, H. (2003). Use of the text messaging in the aftercare of patients with bulimia nervosa. European Eating Disorder Review, 11, 279-290

Beer, R. (2001). Merkmale von Partnerschaftszielen als Veränderungs-Mediatoren in der verhaltenstherapeutischen Kurzzeit-Paartherapie. Universtät Göttingen: unveröffentlichte Dissertation.

Beer, R. \& Breuer, P. (2003). Verhaltenstherapeutische Kurzzeit-Paartherapie von Angesicht zu Angesicht und online: Vergleich der Wirksamkeit zweier Settings. Vortrag, 3. Workshopkongress für Klinische Psychologie und Psychotherapie und 21. Symposium der Fachgruppe Klinische Psychologie und Psychotherapie vom 29.-31.05.2003 in Freiburg. Verfügbar unter: http://wwwuser.gwdg.de/ rbeer/abstract_freiburg_2003.html_[20.05.2007]

Beer, R. \& Breuer, P. (2004). Eheberatung online und Partnerschaftstests online im Projekt Theratalk (www.theratalk.de). Informationsrundschreiben Nr. 208 "Internet @ Beratung" der Deutschen Arbeitsgemeinschaft für Jugend- und Eheberatung e.V., Verfügbar unter: http://www.theratalk.de/pdf/dajeb_artikel_theratalk.pdf [22.05.2007], 24-40

Bieber-Eckardt, U. (2006). 10 Jahre TelefonSeelsorge im Internet - Eine subjektiv gefärbte Rückschau aus sechs Jahren Beteiligung. Auf Draht, Verfügbar unter: http://www.telefonseelsorge.de/aufdraht/aufdraht-001.pdf [22.05.2007], 62

Bolt, D. \& Crawford, R. (2000). Digital Divide: Computers in Our Children's Future. New York, NY: TV Books.

Brähler, E., Scheer, J.W. (1995). Der Gießener Beschwerdebogen (GBB). Testmappe mit 2. ergänzter und revidierter Auflage des Handbuchs. Göttingen: Huber. 
Bremer, C. (2000). Strafbare Internet-Inhalte in internationaler Hinsicht. Ist der Nationalstaat wirklich überholt? Frankfurt: Peter Lang.

Brym, R. J. \& Lenton, R. L. (2001). Love online. A Report on Digital Dating in Canada. Verfügbar unter: http://www.nelson.com/nelson/harcourt/sociology/newsoci-ety 3e/authors/loveonline.pdf [13.04.2007]

Buehlman, K., Gottman, J. M. \& Katz, L. (1992). How a couple views their past predicts their future. Journal of Family Psychology, 5, 295-318

Chen, W. \& Wellman, B. (2003). Charting Digital Divides. Comparing Socioeconomic, Gender, Life Stage, and Rural-Urban Internet Access and Use in Eight Countries. Verfügbar unter: http://www.chass.utoronto.ca/ wellman/publications/amd_ses/ chartingdivides_long.pdf [23.04.2007]

Christl, F. (1998). Beratung im Internet. Erfahrungen der Katholischen Telefonseelsorge. In: Janssen, L. (Hrsg.). Auf der virtuellen Couch, Bonn: Psychiatrie-Verlag, 101-116

Christl, F. (2000). Psychologische Beratung im Internet - Ein Erfahrungsbericht. In: Batinic, B. (Hrsg.): Internet für Psychologen, Göttingen: Hogrefe, 549-565

Daft, R. L. \& Lengel, R. H. (1986). Organizational information reqirements, media richness and structural design. Management Science, 32, 554-571

Daft, R. L., Lengel, R. H. \& Trevino, L. K. (1987). Message equivocality, media selection, and manager performance: Implications for information systems, MIS Quarterly, 11(3), $355-366$

Demmer, U. \& Ludwig, U. (2007). Cybersex im Kinderzimmer. Der Spiegel, 21, 62-63

DiMaggio, P. \& Hargittai, E. (2001). From the 'digital divide' to 'digital inequality': Studying Internet Use as Penetration increases. Princeton: Center for Arts and Cultural Policy Studies, Woodrow Wilson School, Princeton University. 
Döring, N. (1998). Sexuelle Hilfe im Internet. In: Janssen, L. (Hrsg.). Auf der virtuellen Couch. Selbsthilfe, Beratung und Therapie im Internet, Bonn: Psychiatrie-Verlag, 129-157

Döring, N. (2000). Selbsthilfe, Beratung und Therapie im Internet. In Batinic, B. (Hrsg.). Internet für Psychologen (2. Aufl.), Göttingen: Hogrefe, 509-548

Döring, N. \& Eichenberg, C. (2007). Klinisch-psychologische Intervention mit Mobilmedien: Ein neues Praxis- und Forschungsfeld. Psychotherapeut, 52, 127-135

Eichenberg, C. \& Laszig, P. (2003). Internetbasierte Hilfe für Betroffene psychischer Störungen. In Ott, R. \& Eichenberg, C. (Hrsg.). Klinische Psychologie und Internet. Potenziale für die klinische Praxis, Intervention, Psychotherapie und Forschung, Göttingen: Hogrefe, 173-189

Eimeren, B. v. \& Frees, B. (2006). ARD/ZDF-Online-Studie: Schnelle Zugänge, neue Anwendungen, neue Nutzer? Mediaperspektiven 8, 402-415

Filinski, P. (1997). Chatten in der Cyberworld. Bonn: VMI Buch AG.

Fittkau \& Maaß Consulting GmbH (2005). 21. WWW-Benutzer-Analyse W3B. Ergebnisse verfügbar unter: http://www.w3b.org/

Fittkau \& Maaß Consulting GmbH (2004). Kommunikation und Networking in Europa. Erste Studie der Open Business Club GmbH. Ergebnisse verfügbar unter: http://corporate.openbc.com/fileadmin/image_archive/survey_c_Kommunikation_Networking _in_Europa.pdf [10.05.2007]

Franke, G.H. (1995). Die Symptom-Checkliste von Derogatis. Deutsche Version. Göttingen: Beltz Test.

Galanter, M. \& Brook, D. (2001). Network therapy for addiction: bringing family and peer support into office practice. International Journal of Group Psychotherapy, 51, 101-122 
Gehrke, G. (Hrsg.) (2004). Digitale Teilung - digitale Integration: Perspektiven der Internetnutzung. München: kopaed.

Grohol, J. M. (1998). Future clinical directions: professional development, pathology, and psychotherapy on-line. In: Gackenbach, J. (Ed.): Psychology and the Internet. Intrapersonal, interpersonal, and transpersonal implications, San Diego: Academic Press, 111-140

Günther, A. \& Hahn, A. (2000). Suchmaschinen, Robots und Agenten: Informationssuche im WWW. In: Batinic, B. (Hrsg.). Internet für Psychologen, Göttingen: Hogrefe, S. 85-124

Hafner, K. \& Lyon, M. (2000). Arpa Kadabra oder die Geschichte des Internet. Heidelberg: dpunkt.

Hahn, A. \& Günther, A. (1999). Psychologie im Internet: Bestandsaufnahme und Entwicklungstendenzen. In: Batinic, B. (Hrsg.). Internet für Psychologen. Göttingen: Hogrefe.

Hargittai, E. (2002). Second-level digital divide: Differences in people's online skills. First Monday, 7(4).

Herrmann, T. (2001). Kommunikation und Kooperation. In: Schwabe, G., Streitz, N. \& Unland, R. (Hrsg.). CSCW-Kompendium: Lehr- und Handbuch zum computerunterstützten kooperativen Arbeiten. Berlin: Springer, 15-25

Istepanian, R., Laxminarayan, S. \& Pattichis, C. (Eds.) (2006). M-Health. Emerging mobile health systems. Berlin: Springer.

Janssen, L. (1998). Auf der virtuellen Couch. Selbsthilfe, Beratung und Therapie im Internet. In: Janssen, L. (Hrsg.). Auf der virtuellen Couch, Bonn: Psychiatrie-Verlag, 11-25

Johnston, J. M., Leung, G. M., Tin, K. Y. K., Ho, L.-M., Lam, W. \& Fielding, R. (2004). Evaluation of a handheld clinical decision support tool for evidence-based learning and practice in medical undergraduates. Medical Education, 38, 628-627 
Kiesler, S., Siegel, J. \& McGuire, T. (1984). Social psychological aspects of computer mediated communication. American Psychologist, 39, 1123-1134

King, S. A. \& Moreggi, D. (1998). Internet therapy and self help groups - the pros and cons. In: Gackenbach, J. (Ed.). Psychology and the Internet: Intrapersonal, Interpersonal and Transpersonal Implications, San Diego: Academic Press, 77-109

Kleinrock, L. (1961). Information flow in large communication nets. Proposal for a Ph.D. Thesis: Massachusetts Institute of Technology.

Knaevelsrud, C. (2005). Efficiacy of an internet-driven therapy (Interapy) for traumatic stress and the online therapeutic alliance. Universität Zürich: Dissertation.

Knatz, B. (2006). Qualitätsstandards für die Online-Beratung.e-beratungsjournal.net, 2 (1). Verfügbar unter: http://www.e-beratungsjournal.net/ausgabe_0106/knatz.pdf [20.05.2007].

Kordy, H., Hannöver, W. \& Nauer, S. (2003). Das Stuttgart-Heidelberger Modell zur Qualitätssicherung in der statioären Psychotherapie. In: Härter, M., Linster, H. W. \& Stieglitz, R.-D.(Hrsg.) Qualitätsmanagement in der Psychotherapie: Grundlagen, Methoden und Anwendungen, Göttingen: Hogrefe, 289-304

Kordy, H., Golkaramnay, V., Wolf, M., Haug, S. \& Bauer, S. (2006). Internetchatgruppen in Psychotherapie und Psychosomatik - Akzeptanz und Wirksamkeit einer Internet-Brücke zwischen Fachklinik und Alltag. Psychotherapeut, 2, 144-153

Kraut, R., Patterson, M., Lundmark, V., Kiesler, S., Mukopadhyay, T. \& Scherlis, W. (1998). Internet paradox: A social technology that reduces social involvement and psychological well-being? American psychologist, 53, 1017-1031

Lambert, M. J., Hannöver, W., Nisslmüller, K., Richard, M. \& Kordy, H. (2002). Fragebogen zum Ergebnis von Psychotherapie: Zur Reliabilität und Validität der deutschen Übersetzung des Outcome-Questionnaire 45.2 (OQ-45.2). Zeitschrift für Klinische Psychologie und Psychotherapie, 31, 40-47 
Lange, A., Schrieken, B., van de Ven, J.-P., Bredeweg, B., Emmelkamp, P.M.G., van der Kolk, J. Lydsdottir, L., Massaro, M. \& Reuvers, A. (2000). ,INTERAPY': The effects of a short-protocolled treatment of posttraumatic stress and pathological grief through the Internet. Behavioral and Cognitive Psychotherapy, 28 (2), 103-120

Lange, A., van de Ven, J.-P., Schrieken, B. \& Emmelkamp, P. M.G. (2001). Internetmediated, protocol-driven treatment of psychological dysfunction. Journal of Telemedicine and Telecare, 6, 15-21

Lange, A., Rietdijk, D., Hudcovicova, M., van de Ven, J.-P., Schrieken, \& Emmelkamp, P. M. G. (2003). INTERAPY: A controlled randomized trial of the standardized Treatment of posttraumatic stress through the Internet. Journal of Consulting and Clinical Psychology, 71, 901-909

Lange, A., van de Ven, J.-P. , Schrieken, B. \& Smit, M. (2004). 'Interapy' Burn-out: Prävention und Behandlung von Burn-out über das Internet. Verhaltenstherapie, 14, 190-199

Laszig, P. \& Rieg, K. (2004). Psychologische Onlineberatung und -therapie: Internetangebot ersetzt keine klassische Psychotherapie. Deutsches Ärzteblatt, PP 3, 115

Licklider, J.C.R. (1960). Man-Computer Symbioses. IRE Transactions on Human Factors in Electronics, HFE-1, 4-11

Maercker, A. (2005). Evaluation einer Internet-gestützen Behandlung („Interapy“) von posttraumatischen Belastungsstörungen im deutschen Sprachraum. Abschlussbericht. Verfügbar unter: http://www.weisser-ring.de/bundesgeschaeftsstelle/ so_helfen_wir/medizin_psychologie/internet_therapie_fuer_traumatisierte_opfer/interapy_abs chlussbericht.pdf [20.05.2007]

Maercker, A. \& Schützwohl, M. (1998). Erfassung von psychischen Belastungsfolgen: Die Impact of Event Skala - revidierte Version (IES-R) [Assessing the psychological sequelae of stress: The Impact of Event Scale - Revised Version (IES-R)]. Diagnostica, 44, $482-487$ 
Meyer, C. (2006). Deutsche Hauptstelle für Suchtfragen e.V.: Checkliste für Nutzerinnen und Nutzer von Suchhilfeangeboten im Internet. Verfügbar unter: http://www.dhsintern.de/pdf/Checkliste_Endfassung.pdf

Misoch, S. (2006). Online-Kommunikation. Konstanz: UVK

Murphy, L. J. \& Mitchell, D. L. (1998). When writing helps to heal: e-mail as therapy. British Journal of Guidance and Counselling, 26, 21-32

Musch, J. (1997). Die Geschichte des Netzes: ein historischer Abriß. In: Batinic, B. (Hrsg.). Internet für Psychologen. Göttingen: Hogrefe.

Norris, P. (2001). Digital Divide: Falling through the Net: Toward Digital Inclusion, Washington, DC: Cambridge University Press.

Ott, R. (2003). Klinisch-psychologische Intervention und Psychotherapie im Internet: Ein Review zu empirischen Befunden. In Ott, R. \& Eichenberg, C. (Hrsg.). Klinische Psychologie und Internet. Potenziale für die klinische Praxis, Intervention, Psychotherapie und Forschung, Göttingen: Hogrefe, 128-147

Ott,R. \& Eichenberg, C. (2003). Das Internet und die Klinische Psychologie: Analyse der Implikationen. In Ott, R. \& Eichenberg, C. (Hrsg.). Klinische Psychologie und Internet. Potenziale für die klinische Praxis, Intervention, Psychotherapie und Forschung, Göttingen: Hogrefe, 13-18.

Papacharissi, Z. \& Rubin, A. M. (2000). Predictors of internet use. Journal of Broadcasting \& Electronic Media, 44, 175-196

Perse, E. M. \& Courtright, J. A. (1993). Normative images of communication media: Mass and interpersonal channels in the new media environment. Human Communication Research, 19, 485-503

Postman, N. (1992). Das Technopol. Die Macht der Technologien und die Entmündigung der gesellschaft. Frankfurt a. M.: Fischer. 
Postman, N. (1999). Die zweite Aufklärung. Vom 18. ins 21. Jahrhundert. Berlin: BvT.

Rice, R. E. \& Love, G. (1987). Electronic emotion: Socioemotional content in computer mediated communication network. Communication Research, 14, 85-108

Rice, R. E. \& Shook, D. (1990). Relationships of job categories and organizational levels to use of communication channels, including electronic mail: A meta-analysis and extension, Journal of Management Studies, 27(2), 195-229

Robinson, S., Perkins, S., Bauer, S., Hammond, N., Treasure, J. \& Schmidt, U. (2006). Afertcare intervention through text messaging in the treatment of bulimia nervosa: Feasibility plot. International Journal of Eating Disorders, 39(8), 633-638

Schetsche, M. (2004). Internetkriminalität: Daten und Diskurse, Strukturen und Konsequenzen. In: Althoff, M. (Hrsg.). Zwischen Anomie und Inszenierung : Interpretationen der Entwicklung der Kriminalität und der sozialen Kontrolle; zum Gedenken an Detlev Frehsee. Baden-Baden : Nomos.

Schoutrop, M. J. A., Lange, A., Brosschot, J. F. \& Everaerd, W. (1997a). Reprocessing traumatic events by writing assignment: Mechanisms, modes of processing and psychological and physiological effects. (Abstract). Psychosomatic Medicine, 59, 83

Schoutrop, M. J. A. (2000). Structured writing and processing traumatic events: Effects and mechanisms. Dissertation, Universiteit van Amsterdam.

Short, J.A. (1974). Effects of medium of communication on experimental negotiation. Human Relations, 27 (3), 325-334

Short, J.A., Williams, E., \& Christie, B. (1976). The social psychology of telecommunications. New York: John Wiley \& Sons.

Sonnenmoser, M. (2005). Internet-Psychotherapie: Unentdecktes Potenzial. Deutsches Ärzteblatt, PP 4, 84 
Spanier, G. B. (1976). Measuring Dyadic Adjustment: New Scales for Assessing the Quality of Marriage and Similar Dyads. Journal of Marriage and the Family, 38 (1), 15-28

Strotzka, H (1978). Psychotherapie, München , 2. Aufl., 4

Taylor, C. B. \& Luce, K. H. (2003). Computer- and internet-based psychotherapy interventions. Current Directions in Psychological Science, 12, 18-22

Turkle, S. (1995). Life on the screen: Identity in the age of the internet. New York: Simon $\&$ Schuster.

Ungeheuer, G. (1982). Vor-Urteile über Sprechen, Mitteilen, Verstehen. In: Ungeheuer, G. (Hrsg.). Kommunikationstheoretische Schriften I, Aachen: Rader, 229-338

van Well, F. (2000). Psychologische Beratung im Internet: Vergleichende psychologische Untersuchung traditioneller Beratungsangebote mit der Internet-Beratung - unter ergänzender Berücksichtigung der „Internet-Sucht“. Bergisch Gladbach: E. Ferger.

Weiss, D. S. \& Marmar, C. R. (1997). The Impact of Event Scale - Revised. In: Wilson, J. P., Keane, T. M. (Eds.). Assessing psychological trauma and PTSD, 399-411

Wolf, M. \& Kordy, H. (2006). Die therapeutische Beziehung in einem E-Mail-Modell post-stationärer Psychotherapie. Zeitschrift für Psychodynamische Psychotherapie, 3, 137-146

Woodruff, S. I., Edwards, C. C., Conway, T. L. \& Elliott, S. P. (2001). Pilot test of an Internet virtual world chat room for rural teen smokers. Journal of Adolescent Health, 29, $239-243$

Wresch, W. (1996). Disconnected: Haves and Have-Nots in the Information Age. New Brunswick, NJ: Rutgers University Pree.

Yalom. Y.D. (2002). Der Panama-Hut oder Was einen guten Therapeuten ausmacht. München:btb. 


\section{Anhang}

\section{Tabelle A 1: Weltweite Internetnutzung}

(nach http://www.internetworldstats.com/stats.htm; Stand 19. März 2007)

\begin{tabular}{|c|c|c|c|c|c|c|}
\hline Region & Bevölkerung & $\begin{array}{l}\% \text { der } \\
\text { Weltbevölkerung }\end{array}$ & Internetnutzer & $\begin{array}{l}\text { \% der } \\
\text { Bevölkerung } \\
\text { (Penetration) }\end{array}$ & $\begin{array}{l}\% \text { der } \\
\text { Gesamtnutzung }\end{array}$ & Wachstumsrate \\
\hline Afrika & $933,448,292$ & $14.2 \%$ & $33,334,800$ & $3.6 \%$ & $3.0 \%$ & $638.4 \%$ \\
\hline Asien & $3,712,527,624$ & $56.5 \%$ & $398,709,065$ & $10.7 \%$ & $35.8 \%$ & $248.8 \%$ \\
\hline Europa & $809,624,686$ & $12.3 \%$ & $314,792,225$ & $38.9 \%$ & $28.3 \%$ & $199.5 \%$ \\
\hline $\begin{array}{l}\text { Mittlerer } \\
\text { Oasten }\end{array}$ & $193,452,727$ & $2.9 \%$ & $19,424,700$ & $10.0 \%$ & $1.7 \%$ & $491.4 \%$ \\
\hline Nordamerika & $334,538,018$ & $5.1 \%$ & $233,188,086$ & $69.7 \%$ & $20.9 \%$ & $115.7 \%$ \\
\hline $\begin{array}{l}\text { Lateinamerika } \\
\text { / Karibik }\end{array}$ & $556,606,627$ & $8.5 \%$ & $96,386,009$ & $17.3 \%$ & $8.7 \%$ & $433.4 \%$ \\
\hline $\begin{array}{l}\text { Ozeanien / } \\
\text { Australien }\end{array}$ & $34,468,443$ & $0.5 \%$ & $18,439,541$ & $53.5 \%$ & $1.7 \%$ & $142.0 \%$ \\
\hline Insgesamt & $6,574,666,417$ & $100.0 \%$ & $1,114,274,426$ & $16.9 \%$ & $100.0 \%$ & $208.7 \%$ \\
\hline
\end{tabular}




\section{A 2 : Beispiele für Internetseiten}

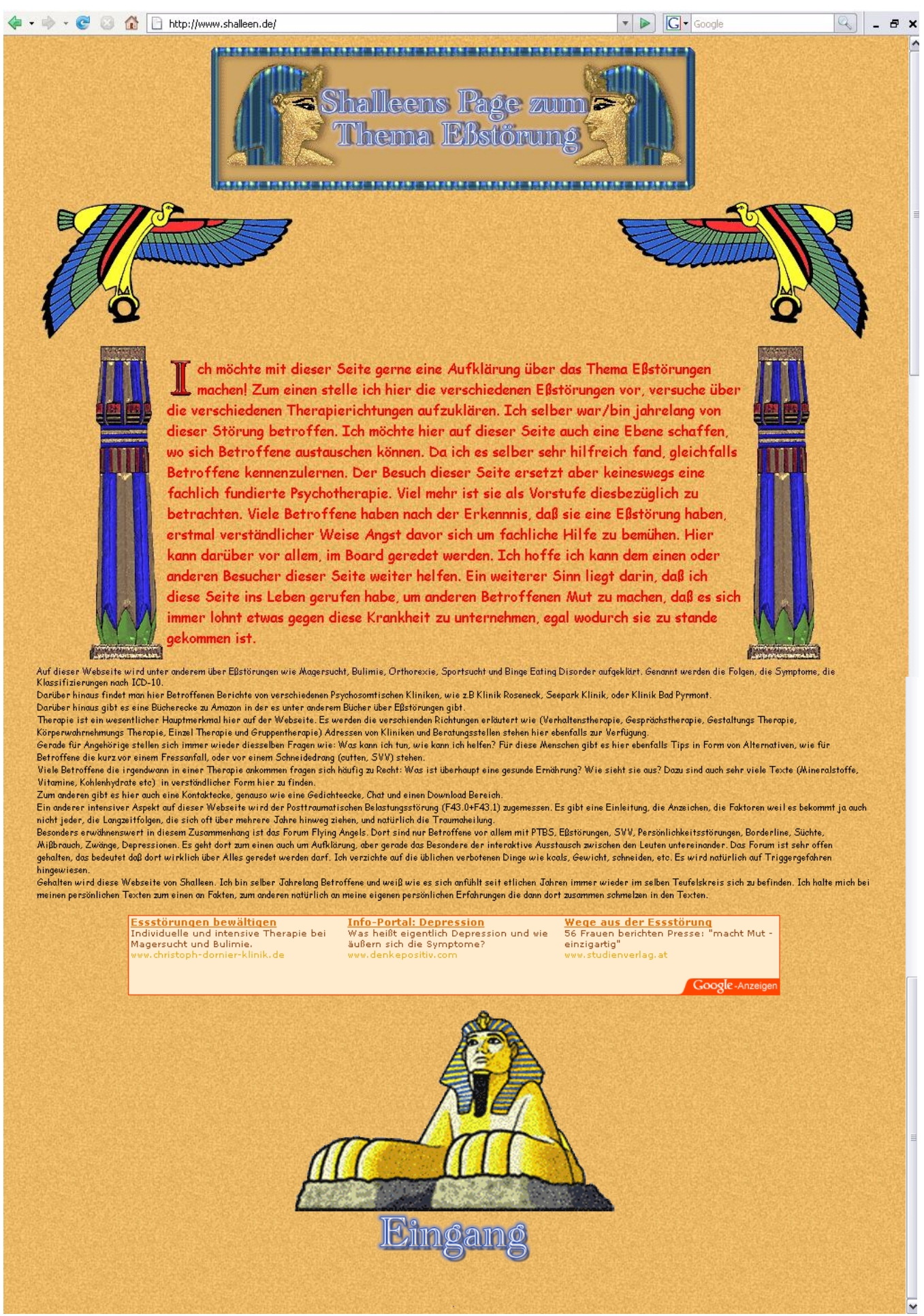

Abbildung A1: Private Internetseite zum Thema Essstörungen

(http://www.shalleen.de/) 


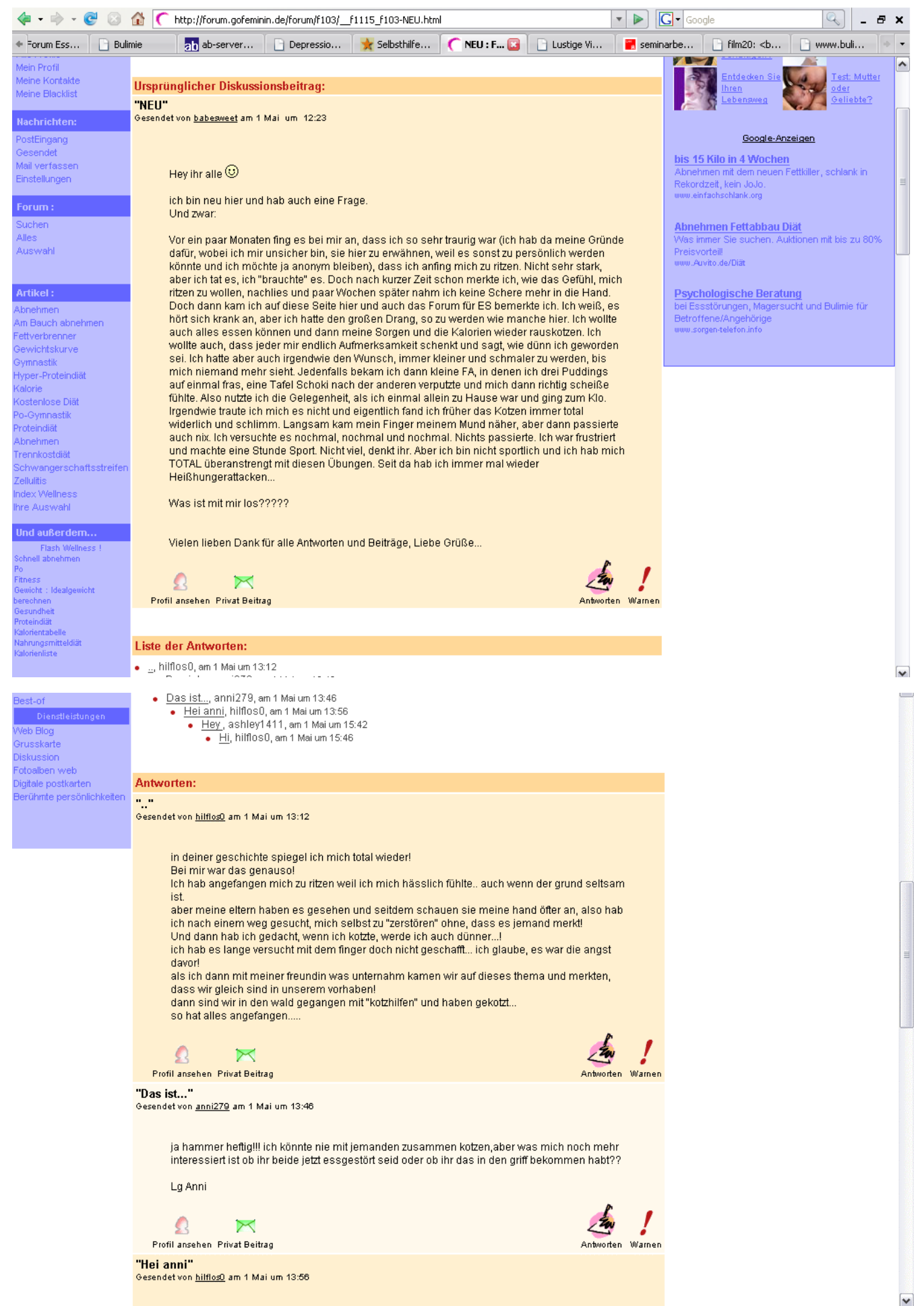

Abbildung A2: Internetforum zum Thema Essstörungen

(http://forum.gofeminin.de/forum/f103/_f1115_f103-NEU.html) 


\section{A 3: Liste zertifizierter Online-Berater}

Quelle: Bundesverband deutscher Psychologinnen und Psychologen

(http://www.bdp-verband.org/service/onlineberater.html)

- www.call-a-tip.de

Nils-Günter Schultze, Psychologischer Psychotherapeut, 60 Jahre, Berlin: Psychologische

E-Mail-Sofort-Beratung; Partnerschaftsprobleme, Erziehungsberatung, persönliche und berufliche Krisen.

- www.detering-brv.de

Jürgen F. Detering: Psychologische Beratung für Lehrerinnen und Lehrer, Psychologische Beratung bei Erziehungs- und Familienproblemen, Supervision, Coaching

- www.kulte.de

Dieter Rohmann: Beratung von Kultmitgliedern, -aussteigern und deren Angehörigen

- www.marionschoemburg.de

Marion Schönburg: Psychotherapie, Mobbingberatung, Coaching, Entspannung

- www.seelenstaerkung.de

Diplom-Psychologe Tim Ziegenhorn, Systemischer Therapeut und Familientherapeut, Ganzheitliche Psychotherapie, Telefon- und Online-Beratung, einfühlsames und humorvolles Knotenlösen

- www.stefan-baier.de

Stefan Baier: Beratung bei Partnerschaftsproblemen, Schwierigkeiten mit Gefühlen, Veränderungen beruflicher Perspektiven, Karrierefragen

- www.webtherapie.info

Prof. Dr. Wilfried Echterhoff: Psychologische Beratung und Therapie bei aktuellen Lebenskrisen und psychischer Erkrankung. Spezialgebiet: Behandlung nach psychotraumatischen Erlebnissen.

\section{- www.wolff-henschen.de}

Wolff Henschen: Psychotherapie, Teamentwicklung, Supervision, Paartherapie 


\title{
A 4: Checkliste für Nutzer von Suchthilfeangeboten im Internet
}

\author{
Deutsche Hauptstelle für Suchtfragen \\ (http://www.dhs-intern.de/pdf/Checkliste_Endfassung.pdf)
}

\section{Checkliste für Nutzerinnen und Nutzer von Suchthilfeangeboten im Internet} Worauf sollen Nutzerinnen und Nutzer achten?

\begin{tabular}{|c|c|c|}
\hline Frage & Antwort & Erläuterungen, warum ist die Frage wichtig \\
\hline $\begin{array}{l}\text { Wie finde ich schnell } \\
\text { die richtige Seite? }\end{array}$ & $\begin{array}{l}\text { Über Suchmaschinen, Fachverbände, Selbsthilfegruppen, Forschungs- } \\
\text { institute, Universitäten, Beratungsstellen, staatliche Institutionen. }\end{array}$ & $\begin{array}{l}\text { Die Adressen finden Sie auf der Rückseite der Checklis- } \\
\text { te. }\end{array}$ \\
\hline $\begin{array}{l}\text { Helfen mir Verlinkun- } \\
\text { gen weiter? }\end{array}$ & $\begin{array}{l}\text { Ja, Sie müssen nicht weiter über Suchmaschinen nach weiterführenden } \\
\text { Texten suchen. Bitte beachten Sie, dass der Betreiber der ersten Web- } \\
\text { seite normalewwelse keine Verantwortung für die Inhalte der verlinkten } \\
\text { Seite übernimmt. In der Regel finden Sie, bevor Sie auf die verlinkte } \\
\text { Seite gehen, eine entsprechende Erklärung (Disclaimer genannt). }\end{array}$ & $\begin{array}{l}\text { Linklisten sind themensortierte Listen, deren Einträge Sie } \\
\text { auf andere Webseiten weiterühren. Typische Links auf } \\
\text { einer Webseite zur Sucht-Selbsthilfe sind: Alkoholselbst- } \\
\text { test (bin ich abhängig?) Alkohol am Arbeitsplat, Leben } \\
\text { ohne Alkohol, Freizeitgestaltung (Angebote von Vereinen } \\
\text { in der Region) usw }\end{array}$ \\
\hline $\begin{array}{l}\text { Ich leide unter einer } \\
\text { Sehbehinderung, kann } \\
\text { ich trotzdem Suchthil- } \\
\text { feseiten nutzen? }\end{array}$ & $\begin{array}{l}\text { Ja, wenn sie barrierefrei gestaltet sind, z.B. } \\
\text { - Ministerium für Arbeit, Soziales, Gesundheit und Familie } \\
\text { whww masgf.brandenburg.de } \\
\text { - Bundeszentrale für gesundheitliche Aufklärung mww.bzga.de }\end{array}$ & $\begin{array}{l}\text { Barrierefrei sind Internetseiten, deren Inform ationen auch } \\
\text { für Menschen mit Behinderungen zugänglich sind, z.B. } \\
\text { über eine vergrößerte Schrift oder akustische Ansage. }\end{array}$ \\
\hline $\begin{array}{l}\text { Woran erkenne ich, wer } \\
\text { sich hinter der Websei- } \\
\text { te verbirgt? }\end{array}$ & $\begin{array}{l}\text { Auf der Webseite solten ein Impressum mit Kontaktdaten (Postan- } \\
\text { schrift, E-Mail, Telefon) und der Webmaster genannt sein. }\end{array}$ & $\begin{array}{l}\text { Der Name des Betreibers kann bereits Auskunft über die } \\
\text { Ausrichtung der Webseite geben. Fehlen diese Angaben, } \\
\text { sollten Sie die Webseite besser meiden. }\end{array}$ \\
\hline $\begin{array}{l}\text { Wie erfahre ich, was der } \\
\text { Anbieter mit seiner } \\
\text { Webseite bezweckt? }\end{array}$ & $\begin{array}{l}\text { Auf der Seite sollten die Ziele und persönliche Motivation, z.B. in einer } \\
\text { Kurzdarstellung oder einem Leitbild erläutert sein. }\end{array}$ & $\begin{array}{l}\text { Es gibt unterschiedliche Motivationen, eine Webseite zu } \\
\text { betreiben, z.B. religiöse, kommerzielle oder ideelle. } \\
\text { Sind die Absichten des Anbieters unklar, sollten Sie die } \\
\text { Webseite besser verlassen. }\end{array}$ \\
\hline $\begin{array}{l}\text { Welche Inhalte (Infor- } \\
\text { mationen und Hilfen) } \\
\text { werden angeboten? }\end{array}$ & $\begin{array}{l}\text { Sie finden je nach Anbieter: } \\
\text { - Erfahrungswissen und persönliche Ansichten von Betroffenen für } \\
\text { Betroffene } \\
\text { - Infos zur Selbsthilfe und Kontakt zu (Online-)Gruppen } \\
\text { - Verzeichnisse von Kliniken und Beratungsstellen } \\
\text { - professionelle Beratungsangebote } \\
\text { - Informationsmaterialien zu Sucht, Drogen, Konsumrisiken etc. } \\
\text { - Alkohol-, Tabak- und Drogenselbsttests } \\
\text { - aktuelle Informationen und Studien zur Suchterkrankung, zu Risiko- } \\
\text { faktoren, zum Verlauf der Erkrankung, zu Behandlungskonzepten etc. } \\
\text { - Alttagstipps (z.B. Rezepte ohne Alkohol) }\end{array}$ & $\begin{array}{l}\text { Sie sollten sich vor der Nutzung des Internet klar sein, } \\
\text { welche Informationen Sie suchen. Im Prinzip können Sie } \\
\text { davon ausgehen, dass Sie zu sämtlichen Fragen Infor- } \\
\text { mationen oder Hinweise im Internet finden. }\end{array}$ \\
\hline $\begin{array}{l}\text { An wen rich } \\
\text { Angebot? }\end{array}$ & $\begin{array}{l}\text { z.B. an } \\
\text { - Alkoholabhängige } \\
\text { - Medikamentenabhängige } \\
\text { - Drogenabhängige } \\
\text { - Spieler } \\
\text { - Angehörige }\end{array}$ & $\begin{array}{l}\text { Auf der Einstiegsseite sollte die Zielgruppe genannt sein. } \\
\text { Wenn sich der Betreiber auf eine Zielgruppe konzentriert, } \\
\text { sind die Informationen oftmals qualitativ besser. Zudem } \\
\text { verhindert eine klare Positionierung der Webseite, dass } \\
\text { Sie unnötig lang suchen müssen. }\end{array}$ \\
\hline $\begin{array}{l}\text { Wird die Webseite von } \\
\text { privaten Unternehmen } \\
\text { finanziert (gesponsert)? }\end{array}$ & $\begin{array}{l}\text { Webseiten von gemeinnütigen und staatlichen Beratungsstellen oder } \\
\text { Selbsthiffeverbänden werden in der Regel nicht gesponsert. Kommer- } \\
\text { zielle und nicht verbandlich organisierte Selbsthilfe-Anbieter werden } \\
\text { teilweise von Dritten finanziert. }\end{array}$ & $\begin{array}{l}\text { Es muss erkennbar sein, wer die Webseite finanziert und } \\
\text { welche Interessen die Geldgeber damit verfolgen: Das } \\
\text { Interesse der Geldgeber kann einen Einfluss auf die } \\
\text { Ausgewogenheit der Informationen haben, z.B. Konsum- } \\
\text { risiken beschönigen. }\end{array}$ \\
\hline $\begin{array}{l}\text { Was bietet mir die } \\
\text { Onlineberatung (E-Mail- } \\
\text {, Chat-, Foren- } \\
\text { Beratung)? }\end{array}$ & $\begin{array}{l}\text { - Zu jeder Zeit Fragen stellen bzw. Kontakt zu einem Berater aufneh- } \\
\text { men zu können } \\
\text { - eine Antwort nach kurzer Zeit } \\
\text { - räumliche Unabhängigkeit } \\
\text { - Anonymität und Schweigepflicht der Berater }\end{array}$ & $\begin{array}{l}\text { Die Onlineberatung empfiehlt sich vor allem für Men- } \\
\text { schen, die sich gerne schriftlich austauschen. } \\
\text { Das Formulieren der Nachricht an den Berater führt } \\
\text { bereits zu einer vertieften Auseinandersetzung mit der } \\
\text { Suchtproblematik, die schriftlichen Antworten können } \\
\text { wiederholt gelesen werden. }\end{array}$ \\
\hline $\begin{array}{l}\text { Ist eine Onlineberatung } \\
\text { kostenlos? }\end{array}$ & $\begin{array}{l}\text { Gemeinnützige und staatliche Beratungsstellen, Initiativen sowie Ange- } \\
\text { bote der Selbsthilfe sind kostenlos. } \\
\text { Kommerzielle Angebote sind häufig kostenpflichtig, z.B. } 30,00 € \text { pro E- } \\
\text { Mailantwort. }\end{array}$ & $\begin{array}{l}\text { Wichtig ist vor allem, dass Sie vor Inanspruchnahme } \\
\text { einer Dienstleistung wissen, was sie kosten wird. Die } \\
\text { Kosten für eine Onlineberatung werden von den Kran- } \\
\text { kenkassen nicht übernommen. }\end{array}$ \\
\hline $\begin{array}{l}\text { Was bietet mir die } \\
\text { professionelle Online- } \\
\text { beratung? }\end{array}$ & $\begin{array}{l}\text { - Beratung zu rechtlichen, medizinischen, psychologischen und/oder } \\
\text { pädagogischen Fragestellungen } \\
\text { - Hilfen bei Krisen }\end{array}$ & $\begin{array}{l}\text { Sie sollten darauf achten, dass der Berater bei fachspe- } \\
\text { zifischen Fragestellungen auch über eine entsprechende } \\
\text { Qualifikation (Art, Jurist usw.) verügt. }\end{array}$ \\
\hline $\begin{array}{l}\text { Was bietet mir die } \\
\text { Onlineberatung der } \\
\text { Selbsthilfe? }\end{array}$ & $\begin{array}{l}\text { - Austausch von persönlichen Erfahrungen von Betroffenen für andere } \\
\text { Betroffene } \\
\text { - ehrenamtliche Berater (Betroffene), die z.T. auch über eine Qualifika- } \\
\text { tion als freiwillige Suchtkrankenhelfer o.ä. verfügen }\end{array}$ & $\begin{array}{l}\text { Fach-Beratungen werden z.T. auch von professionellen } \\
\text { Mitarbeitern (hauptamtlich tätig) der Abstinenz- und } \\
\text { Selbsthilfeverbände, z.B. der Guttempler oder des } \\
\text { Kreuzbundes durchgeführt. }\end{array}$ \\
\hline Worauf sollte ich bei & - auf die Auswahl des Beraters (Vertrauenswürdigkeit) & Die Onlineberatung unterliegt keinen staatlichen Kontrol- \\
\hline
\end{tabular}




\begin{tabular}{|c|c|c|}
\hline $\begin{array}{l}\text { der E-Mailberatung } \\
\text { achten? }\end{array}$ & $\begin{array}{l}\text { - seine Qualifikation (Erfahrung und Ausbildung) } \\
\text { - ob er in einer anerkannten Einrichtung arbeitet }\end{array}$ & $\begin{array}{l}\text { len. Theoretisch kann jeder, der über einen Internetauf- } \\
\text { tritt verfügt, Onlineberatung anbieten. Deshalb sollten Sie } \\
\text { Angaben zum Berater (z.B. beruflicher Werdegang) } \\
\text { vorfinden. }\end{array}$ \\
\hline $\begin{array}{l}\text { Worauf sollte ich im } \\
\text { Chat achten? }\end{array}$ & $\begin{array}{l}\text { - dass der Chat nur angemeldete Personen zulässt } \\
\text { - dass Sie einen Chat nie mit dem eigenen Namen betreten } \\
\text { - dass Sie einen beobachteten Chat mit definierten Öffnungszeiten } \\
\text { vorziehen sollten } \\
\text { - dass die Beiträge zumeist von Laien geschrieben sind und auch } \\
\text { falsche Informationen verbreitet werden können } \\
\text { - ob der Chatbetreiber Benimmregeln vorgegeben hat }\end{array}$ & $\begin{array}{l}\text { Fehlen Anmeldemodalitäten, sollten Sie besser auf das } \\
\text { Chatangebot dieser Webseite verzichten. } \\
\text { Im beobachteten Chat ist zumindest ein Operator }{ }^{3} \text { anwe- } \\
\text { send, der Sie vor Störern schützen kann. } \\
\text { Empfehlenswert ist die Venwendung der Chatsprache } \\
\text { (vgl. } \\
\text { http//Wwww.freenet.de/hilfe/dienste/community/freenetcha } \\
\text { V/abkuerzungen/) }\end{array}$ \\
\hline $\begin{array}{l}\text { Worauf sollte ich im } \\
\text { Forum achten? }\end{array}$ & $\begin{array}{l}\text { - Beiträge werden von Laien formuliert und ergänzt } \\
\text { - ob der Forumanbieter darauf hinweist, dass die Beiträge gegengele- } \\
\text { sen, ggf. zensiert werden } \\
\text { - ob Schutzmechanismen (z.B. Anmeldung und Passwort) vorgesehen } \\
\text { sind. }\end{array}$ & $\begin{array}{l}\text { Foren bieten wenig Anonymität und Schut vor uner- } \\
\text { wünschten Lesern. } \\
\text { In einigen Foren sind neben Ihrem geschrieben Beitrag, } \\
\text { Ihr Vorname und Ihre E-Mailadresse für alle Leser er- } \\
\text { kennbar. Die Anonymität ist nicht gewährleistet. }\end{array}$ \\
\hline $\begin{array}{l}\text { Worauf sollte ich bei } \\
\text { Fachtexten achten? }\end{array}$ & $\begin{array}{l}\text { - auf Angaben zur Urheberschaft (Autor), Quellenangaben und Datum } \\
\text { der Textveröffentlichung }\end{array}$ & $\begin{array}{l}\text { Fehlen Quellengaben, ist nicht erkennbar, ob es sich um } \\
\text { Fachwissen oder Laienmeinung handelt. } \\
\text { Die Gefahr, dass auch veraltete Informationen weiterge- } \\
\text { geben werden, ist bei der Venwendung von Fremdtexten } \\
\text { (Beiträge anderer Autoren) groß. }\end{array}$ \\
\hline $\begin{array}{l}\text { Verfügen Suchthilfesei- } \\
\text { ten über ein Qualitäts- } \\
\text { siegel? }\end{array}$ & $\begin{array}{l}\text { Es gibt kein spezielles Qualitätssiegel für Suchthilfeseiten. Für Websei- } \\
\text { ten mit Gesundheitsthemen gibt es im deutschsprachigen Raum zwei } \\
\text { verbreitete Qualitätssysteme: AFGIS (wMm.afgis.de) und Health On Net } \\
\text { (www.hon.ch). Uns sind bisher keine Suchthilfe-Angebote bekannt, die } \\
\text { sich an einem dieser beiden Qualitätssysteme orientieren. }\end{array}$ & $\begin{array}{l}\text { Qualitätssiegel auf der Homepage zeigen, dass der } \\
\text { Anbieter seine Webseite nach den Vorgaben der Quali- } \\
\text { tätssysteme erstellt und dieses auch überprüfen lässt. } \\
\text { Solche geprüften Webseiten sind seriös und können } \\
\text { unbedenklich genutzt werden. }\end{array}$ \\
\hline $\begin{array}{l}\text { Wie werden meine } \\
\text { Daten geschützt? }\end{array}$ & $\begin{array}{l}\text { Der Anbieter sollte darlegen, wie die persönlichen Daten der Nutzenden } \\
\text { geschützt werden. }\end{array}$ & $\begin{array}{l}\text { Sie sollten sich immer genau überlegen, ob die weiter- } \\
\text { gegebenen Informationen dem angebotenen Persönlich- } \\
\text { keitsschutz entsprechen. }\end{array}$ \\
\hline $\begin{array}{l}\text { Wie sicher sind meine } \\
\text { Daten bei der Onlinebe- } \\
\text { ratung? }\end{array}$ & $\begin{array}{l}\text { Die Vertraulichkeit ist mit den gängigen E-Mail-Programmen nicht } \\
\text { gewährleistet. } \\
\text { Kommunikationsangebote auf einer Webseite können mehr Sicherheit } \\
\text { bieten (gleiche Technik wie beim e-Banking). }\end{array}$ & $\begin{array}{l}\text { Obwohl die Internetkommunikation nicht besonders gut } \\
\text { geschützt ist, sind uns keine Missbrauchsvorfälle be- } \\
\text { kannt. }\end{array}$ \\
\hline
\end{tabular}




\section{A 5: Auswahl deutschsprachiger Online-Beratungsangebote}

\begin{tabular}{|c|c|c|c|}
\hline Anbieter / URL & Kosten & Medium & Vorgehen \\
\hline $\begin{array}{l}\text { Christian Hilscher } \\
\text { Dipl.-Psychologe \& Heilpraktiker der } \\
\text { Psychotherapie } \\
\text { http://www.onlineberatung-therapie.de/ }\end{array}$ & $\begin{array}{l}\text { 40,- } \\
\text { Euro/Mail } \\
1,49 \\
\text { Euro/Minute }\end{array}$ & $\begin{array}{l}\text { E-Mail } \\
\text { Telefon }\end{array}$ & $\begin{array}{l}\text { Problemschilderu } \\
\text { ng; Antwort max. } \\
24 \text { Std. nach } \\
\text { Zahlungseingang }\end{array}$ \\
\hline $\begin{array}{l}\text { Dipl.- Psychologin Christiane } \\
\text { Turnheim } \\
\text { http://www.psychohelp.at/ }\end{array}$ & $\begin{array}{l}\text { 25,- EUR (1. } \\
\text { Mail), } \\
\text { danach 20,- } \\
\text { EUR/Mail }\end{array}$ & E-Mail & $\begin{array}{l}\text { Problemschilderu } \\
\text { ng, Antwort } \\
\text { baldmöglichst }\end{array}$ \\
\hline $\begin{array}{l}\text { Swen Heidenreich } \\
\text { Dipl.-Psychologe, Trainer, Coach } \\
\text { http://www.psychologischeberatung.inf } \\
\text { o/ }\end{array}$ & k. A. & $\begin{array}{l}\text { E-Mail } \\
\text { Telefon } \\
\text { Praxister } \\
\text { min }\end{array}$ & k. A. \\
\hline $\begin{array}{l}\text { Stefan Baier } \\
\text { Dipl.-Psychologe, Psycholog. Psycho- } \\
\text { therapeut, Supervisor und Coach } \\
\text { http://www.stefan- } \\
\text { baier.de/beratung/index.html }\end{array}$ & $\begin{array}{l}\text { 50,- EUR/ } \\
\text { Beratung } \\
\text { (max. } 5 \\
\text { Mails), } \\
\text { ausführliche } \\
\text { Beratung } \\
\text { nach } \\
\text { Absprache }\end{array}$ & E-Mail & $\begin{array}{l}\text { Anmeldung, erste } \\
\text { Problemschilderu } \\
\text { ng, Antwort } \\
\text { innerhalb von } \\
\text { max. } 3 \text { Tagen }\end{array}$ \\
\hline $\begin{array}{l}\text { Horst Krüger } \\
\text { Dipl.-Sozialarbeiter } \\
\text { Heilpraktiker / Psychotherapie } \\
\text { http://www.pb-id.homepage.t- } \\
\text { online.de/impr.htm }\end{array}$ & $\begin{array}{l}\text { 1 Email: } \\
\text { 20,- EUR } \\
4 \text { Emails: } \\
\text { 85,- EUR } \\
10 \text { E-Mails: } \\
\text { 210,- EUR }\end{array}$ & E-Mail & k. A. \\
\hline http://www.seelsorge.net/ & kostenlos & $\begin{array}{l}\text { E-Mail } \\
\text { SMS }\end{array}$ & $\begin{array}{l}\text { E-Mail, } \\
\text { schnellstmögliche } \\
\text { Beantwortung }\end{array}$ \\
\hline
\end{tabular}




\section{A 6: Liste der im Literatur-Review berücksichtigten Studien}

(Ott, 2003, S.133: Studien zu Angst- und Panikstörungen)

\begin{tabular}{|c|c|c|c|}
\hline Studie & Intervention & Stichprobe, Design & Ergebnisse \\
\hline $\begin{array}{l}\text { Bouchard, } \\
\text { Payeur, Ri- } \\
\text { vard, Allard, } \\
\text { Paquin, } \\
\text { Renaud \& } \\
\text { Goyer } \\
(2000)\end{array}$ & $\begin{array}{l}\text { 12h Video-Konferenz, } \\
\text { ausgebildete } \\
\text { Therapeuten, kognitiv- } \\
\text { behaviorales Material }\end{array}$ & $\begin{array}{l}\text { UV1: Patn. Mit } \\
\text { Panikstörung und } \\
\text { Agoraphobie (n=12), } \\
\text { keine KG } \\
\text { UV2: Zeitpunkt, prä vs. } \\
\text { post } \\
\text { AV: standardisierte } \\
\text { Fragebögen und } \\
\text { klinische Interviews }\end{array}$ & $\begin{array}{l}\text { Signifikante } \\
\text { Verbesserung der } \\
\text { Paniksymptomatik } \\
\text { (Anzahl und Intensität } \\
\text { der Attacken, } \\
\text { Kontrollerwartung etc.) } \\
\text { Signifikante } \\
\text { Verbesserung des } \\
\text { allgemeinen Befindens } \\
\text { Positive Aussagen zur } \\
\text { therapeutischen Allianz }\end{array}$ \\
\hline $\begin{array}{l}\text { Carlbring, } \\
\text { Westling, } \\
\text { Ljungstrand, } \\
\text { Ekselius \& } \\
\text { Andersson } \\
\text { (2001) }\end{array}$ & $\begin{array}{l}\text { WWW-Angebot mit } \\
\text { kognitiv-behavioralen } \\
\text { Komponenten } \\
\text { (Psychoedukation, } \\
\text { interozeptive } \\
\text { Exposition, etc.), } \\
\text { Einweisung per E-Mail }\end{array}$ & $\begin{array}{l}\text { UV1: Patn. Mit } \\
\text { Panikstörung: Internet- } \\
\text { EG (n=13) vs. f2f-KG1 } \\
(n=14) \text { vs. Warte-KG2 } \\
(n=14) \\
\text { UV2: Zeitpunkt, prä vs. } \\
\text { post } \\
\text { AV: Fragebogen }\end{array}$ & $\begin{array}{l}\text { Signifikante } \\
\text { Verbesserung der } \\
\text { Angstsymptomatik in } \\
\text { der EG nd der f2f-KG } \\
\text { (Anzahl der Symptome, } \\
\text { Anzahl u. Intensität der } \\
\text { Attacken, etc.) }\end{array}$ \\
\hline $\begin{array}{l}\text { Klein \& } \\
\text { Richards } \\
(2001)\end{array}$ & $\begin{array}{l}\text { WWW-Angebot mit } \\
\text { kognitiv-behavioralen } \\
\text { Komponenten, } \\
\text { einwöchig, } \\
\text { einwöchiges self- } \\
\text { monitoring-Training } \\
\text { für beide Gruppen } \\
\text { vorab }\end{array}$ & $\begin{array}{l}\text { UV1: Patn. Mit } \\
\text { Panikstörung, Internet- } \\
\text { EG (n=11) vs. self- } \\
\text { monitoring-KG (n=11) } \\
\text { UV2: Zeitpunkt, prä vs. } \\
\text { post vs. 1-Woche- } \\
\text { follow-up } \\
\text { AV: Fragebögen zur } \\
\text { Angst- und allgemeinen } \\
\text { Symptomatik }\end{array}$ & $\begin{array}{l}\text { Signifikante } \\
\text { Verbesserung der } \\
\text { Angstsymptomatik in } \\
\text { der EG } \\
\text { Keine Verbesserung in } \\
\text { begleitenden } \\
\text { depressiven } \\
\text { Symptomen }\end{array}$ \\
\hline $\begin{array}{l}\text { Richards \& } \\
\text { Alvarenga } \\
(2002)\end{array}$ & $\begin{array}{l}\text { WWW-Angebot mit } \\
\text { kognitiv-behavioralen } \\
\text { Komponenten, }\end{array}$ & $\begin{array}{l}\text { UV1: Patn. Mit } \\
\text { Panikstörung ( } \mathrm{n}=9=\text {, } \\
\text { keine KG }\end{array}$ & $\begin{array}{l}\text { Signifikante } \\
\text { Verbesserung der } \\
\text { Paniksymptomatik }\end{array}$ \\
\hline
\end{tabular}




\begin{tabular}{|l|l|l|l|}
\hline achtwöchiges & UV2: Zeitpunkt, prä vs. & (Intensität der \\
& post vs. 3-Monats & Attacken, \\
& follow-up & katastrophisierende \\
& AV: Fragebögen zur & Gedanken) \\
& Paniksymptomatik & Keine Verbesserung \\
& & der \\
& & Körperaufmerksamkeit \\
\hline
\end{tabular}


(Ott, 2003, S. 134: Studien zu depressiven Störungen)

\begin{tabular}{|c|c|c|c|}
\hline Studie & Intervention & Stichprobe, Design & Ergebnisse \\
\hline $\begin{array}{l}\text { Christensen, } \\
\text { Griffiths \& } \\
\text { Korten } \\
(2002)\end{array}$ & $\begin{array}{l}\text { WWW-Angebot mit } \\
\text { kognitiv-behavioralen } \\
\text { Modulen (kognitive } \\
\text { Umstrukturierung, } \\
\text { Aktivitätsplanung), } \\
\text { präventiver Charakter }\end{array}$ & $\begin{array}{l}\text { UV1: subklinische } \\
\text { Stichprobe, Nutzer der } \\
\text { Seite (n=2909), keine } \\
\text { KG } \\
\text { UV2: Zeitpunkt, prä vs. } \\
\text { post } \\
\text { AV: Fragebogen }\end{array}$ & $\begin{array}{l}\text { Signifikante Reduktion } \\
\text { der depressiven } \\
\text { Symptomatik } \\
\text { Signifikante Reduktion } \\
\text { der Angstsymptomatik }\end{array}$ \\
\hline $\begin{array}{l}\text { Clarke, } \\
\text { Reid, } \\
\text { Eubanks, } \\
\text { O’Connor, } \\
\text { DeBar, Kel- } \\
\text { leher \& } \\
\text { Nunley } \\
(2002)\end{array}$ & $\begin{array}{l}\text { WWW-Angebot mit } \\
\text { kognitiven und } \\
\text { psychoedukativen } \\
\text { Modulen }\end{array}$ & $\begin{array}{l}\text { UV1: Patienten mit } \\
\text { depressiven Störungen: } \\
\text { Internet-EG (n0144) vs. } \\
\text { Warte-KG (n=155) } \\
\text { UV2: Zeitpunkt, prä vs. } \\
\text { post vs. follow-up } \\
\text { (4,6,16 u. } 32 \text { Wochen) } \\
\text { AV: Fragebogen }\end{array}$ & $\begin{array}{l}\text { Keine Reduktion der } \\
\text { depressiven } \\
\text { Symptomatik im } \\
\text { Vergleich zwischen EG } \\
\text { und KG } \\
\text { Subgruppenbildung: } \\
\text { Patn. Mit leichter und } \\
\text { schwerer Symptomatik, } \\
\text { signifikante Reduktion } \\
\text { der depressiven } \\
\text { Symptome für } \\
\text { Patienten mit leichter } \\
\text { Symptomatik }\end{array}$ \\
\hline $\begin{array}{l}\text { Nelson, } \\
\text { Barnard \& } \\
\text { Cain (2003) }\end{array}$ & $\begin{array}{l}\text { Video-Konferenz, 8- } \\
\text { wöchig, ausgebildete } \\
\text { Therapeuten, kognitiv- } \\
\text { behaviorales Manual }\end{array}$ & $\begin{array}{l}\text { UV!. Jugendliche } \\
\text { Patienten mit } \\
\text { depressiven Störungen: } \\
\text { Video-EG } 8 \mathrm{n}=14 \text { ) vs. } \\
\text { f2f-KG (n=155) } \\
\text { UV2: Zeitpunkt, prä vs. } \\
\text { post } \\
\text { AV: standardisierte } \\
\text { Fragebögen }\end{array}$ & $\begin{array}{l}\text { Signifikante Reduktion } \\
\text { der depressiven } \\
\text { Symptomatik in beiden } \\
\text { Gruppen } \\
\text { Keine } \\
\text { Gruppenunterschiede }\end{array}$ \\
\hline $\begin{array}{l}\text { Patten } \\
\text { (2003) }\end{array}$ & $\begin{array}{l}\text { WWW-Angebot mit } \\
\text { psychoedukativen } \\
\text { Modulen, präventiver } \\
\text { Charakter }\end{array}$ & $\begin{array}{l}\text { UV1: subklinische } \\
\text { Stichprobe; } \\
\text { Ratsuchende: Internet- } \\
\text { EG (n=390) vs. Warte- }\end{array}$ & $\begin{array}{l}\text { Keine Verbesserung } \\
\text { der depressiven } \\
\text { Symptomatik } \\
\text { Keine }\end{array}$ \\
\hline
\end{tabular}




\begin{tabular}{|l|l|l|}
\hline & KG (n=396) & Gruppenunterschiede \\
& UV2: Zeitpunkt, prä vs. & \\
& post vs. 3-Monats & \\
& follow-up & \\
& AV: standardisierte & \\
& Fragebögen & \\
\hline
\end{tabular}


(Ott, 2003, S. 135: Studien z Essstörungen)

\begin{tabular}{|c|c|c|c|}
\hline Studie & Intervention & Stichprobe, Design & Ergebnisse \\
\hline $\begin{array}{l}\text { Celio, } \\
\text { Winzelberg, } \\
\text { Wilfley, } \\
\text { Eppstein- } \\
\text { Herald, } \\
\text { Springer, } \\
\text { Dev\& Taylor } \\
(2000)\end{array}$ & $\begin{array}{l}\text { WWW-Angebot und E- } \\
\text { Mail Versand mit } \\
\text { kognitiv-behavioralen } \\
\text { Modulen * }\end{array}$ & $\begin{array}{l}\text { UV1: subklinische } \\
\text { Stichprobe: Internet- } \\
\text { EG (n=25) vs f2f-KG1 } \\
\text { (n=26) vs. Warte-KG2 } \\
\text { UV2: Zeitpunkt, prä vs. } \\
\text { post vs. 4-Monats } \\
\text { follow-up } \\
\text { AV: FB zu } \\
\text { Essverhalten u. - } \\
\text { einstellung und } \\
\text { Körperbild }\end{array}$ & $\begin{array}{l}\text { Signifikante Reduktion } \\
\text { des negativen } \\
\text { Essverhaltens in der } \\
\text { EG und KG1 } \\
\text { Signifikante } \\
\text { Verbesserung des } \\
\text { Körperbildes in der EG } \\
\text { und KG1 } \\
\text { Keine Unterschiede } \\
\text { zwischen EG und KG1 }\end{array}$ \\
\hline $\begin{array}{l}\text { Robinson \& } \\
\text { Serfaty (2001) }\end{array}$ & $\begin{array}{l}\text { E-Mail- } \\
\text { Beratungsangebot mit } \\
\text { kognitiv-behavioralen } \\
\text { Inhalten, durchgeführt } \\
\text { von } 2 \\
\text { Psychotherapeuten }\end{array}$ & $\begin{array}{l}\text { UV1: Patn. Mit } \\
\text { Bulimie und Binge } \\
\text { Eating Störung: } \\
\text { Internet-EG (n=19), } \\
\text { keine KG } \\
\text { UV2: Zeitpunkt, prä vs. } \\
\text { post vs. 3-Monats } \\
\text { follow-up } \\
\text { AV: FB zum } \\
\text { Essverhalten, } \\
\text { Compliance } \\
\text { Fragebogen }\end{array}$ & $\begin{array}{l}\text { Signifikante Reduktion } \\
\text { des symptomatischen } \\
\text { Verhaltens und der } \\
\text { Kognitionen } \\
\text { Signifikante positive } \\
\text { Korrelation zwischen } \\
\text { Länge der E-Mails, } \\
\text { Zufriedenheit und } \\
\text { Symptomreduktion }\end{array}$ \\
\hline $\begin{array}{l}\text { Winzelberg, } \\
\text { Eppstein, } \\
\text { Eldredge, } \\
\text { Wilfley, } \\
\text { Dasmahapatra, } \\
\text { Dev \& Taylor } \\
(2000)\end{array}$ & $\begin{array}{l}\text { WWW-Angebot und E- } \\
\text { Mail Versand mit } \\
\text { kognitiv-behavioralen } \\
\text { Modulen * }\end{array}$ & $\begin{array}{l}\text { UV1: subklinische } \\
\text { Stichprobe: Internet- } \\
\text { EG ( } n=30) \text { vs. Warte- } \\
\text { KG (n=30) } \\
\text { UV2: Zeitpunkt, prä vs. } \\
\text { post vs. 3-Monats } \\
\text { follow-up } \\
\text { AV: FB zu } \\
\text { Essverhalten u. - } \\
\text { einstellung und }\end{array}$ & $\begin{array}{l}\text { Signifikante } \\
\text { Verbesserung des } \\
\text { Körperbildes in EG im } \\
\text { Vgl. zur KG } \\
\text { Signifikante Reduktion } \\
\text { des Bedürfnisses } \\
\text { schlanker zu werden in } \\
\text { EG }\end{array}$ \\
\hline
\end{tabular}




\begin{tabular}{|c|c|c|c|}
\hline & & Körperbild & \\
\hline $\begin{array}{l}\text { Winzelberg, } \\
\text { Taylor, } \\
\text { Sharpe, El- } \\
\text { dredge, Dev \& } \\
\text { Constatinous } \\
\text { (1998) }\end{array}$ & $\begin{array}{l}\text { WWW-Angebot und E- } \\
\text { Mail Versand mit } \\
\text { kognitiv-behavioralen } \\
\text { Modulen }\end{array}$ & $\begin{array}{l}\text { UV1: subklinische } \\
\text { Stichprobe: Internet- } \\
\text { EG ( } n=27) \text { vs. Warte- } \\
\text { KG (n=30) } \\
\text { UV2: Zeitpunkt, prä vs. } \\
\text { post vs. 3-Monats } \\
\text { follow-up } \\
\text { AV: FB zu } \\
\text { Essverhalten u. - } \\
\text { einstellung und } \\
\text { Körperbild }\end{array}$ & $\begin{array}{l}\text { Signifikante } \\
\text { Verbesserung innerhalb } \\
\text { der EG in allen } \\
\text { Fragebogenmaßen } \\
\text { Im Vergleich zur KG } \\
\text { nur signifikante } \\
\text { Verbesserung des } \\
\text { Körperbildes }\end{array}$ \\
\hline $\begin{array}{l}\text { Zabinski, } \\
\text { Pung, Wilfley, } \\
\text { Eppstein, } \\
\text { Winzelberg, } \\
\text { Celio \& } \\
\text { Taylor (2001) }\end{array}$ & $\begin{array}{l}\text { WWW-Angebot und E- } \\
\text { Mail Versand mit } \\
\text { kognitiv-behavioralen } \\
\text { Modulen * }\end{array}$ & $\begin{array}{l}\text { UV1: subklinische } \\
\text { Stichprobe: Internet- } \\
\text { EG (n=28) vs. Warte- } \\
\text { KG (n=29) } \\
\text { UV2: Zeitpunkt, prä vs. } \\
\text { post vs. 10-Wochen } \\
\text { follow-up } \\
\text { AV: FB zu } \\
\text { Essverhalten u. - } \\
\text { einstellung und } \\
\text { Körperbild }\end{array}$ & $\begin{array}{l}\text { Signifikante } \\
\text { Verbesserung der } \\
\text { zentralen } \\
\text { Fragebogenmaße in } \\
\text { beiden Gruppen } \\
\text { Keine Gruppeneffekte }\end{array}$ \\
\hline
\end{tabular}

(Ott, 2003, S. 137: Studien zu Adipositas)

\begin{tabular}{|c|c|c|c|}
\hline Studie & Intervention & Stichprobe, Design & Ergebnisse \\
\hline $\begin{array}{l}\text { Harvey-Berino, } \\
\text { Pintauro \& Gold } \\
(2002)\end{array}$ & $\begin{array}{l}\text { WWW-Angebot, } \\
\text { diskussionsforen und Chat } \\
\text { mit psychoedukativen } \\
\text { Modulen, 22Wochen }\end{array}$ & $\begin{array}{l}\text { UV1: Patn. Mit } \\
\text { Adipositas: } \\
\text { Internet-EG }(n=13) \\
\text { vs. f2f-KG1 }(n=15) \\
\text { vs. Warte-KG2 } \\
(n=15) \\
\text { UV2: Zeitpunkt, } \\
\text { prä vs. post }\end{array}$ & $\begin{array}{l}\text { Signifikante } \\
\text { gewichtsreduktion in EG } \\
\text { und KG1 } \\
\text { Keine Unterschiede } \\
\text { zwischen EG und KG1 } \\
\text { Signifikant höhere } \\
\text { Behandlungszufriedenheit }\end{array}$ \\
\hline
\end{tabular}




\begin{tabular}{|c|c|c|c|}
\hline & & $\begin{array}{l}\text { AV: Gewicht, } \\
\text { Fragebogen zum } \\
\text { Essvrhalten sowie } \\
\text { Compliance }\end{array}$ & und Compliance in KG1 \\
\hline $\begin{array}{l}\text { Harvey-Berino, } \\
\text { Pintauro, } \\
\text { Buzzell, } \\
\text { DiGiulio, Casey, } \\
\text { Gold, Moldovan } \\
\text { \& Ramirez } \\
\text { (2002) }\end{array}$ & $\begin{array}{l}\text { 6-monatiges f2f-Programm } \\
\text { mit überwiegend edukativen } \\
\text { Anteilen, 12-monatiges } \\
\text { Aufrechterhaltungsprogramm } \\
\text { über WWW-Angebot }\end{array}$ & $\begin{array}{l}\text { UV1: Patn. Mit } \\
\text { Adipositas: } \\
\text { Internet-EG (n=32) } \\
\text { vs. f2f-KG1 mit } \\
\text { häufigem Kontakt } \\
\text { vs. f2f-KG2 mit } \\
\text { minimalem Kontakt } \\
\text { (n=32) } \\
\text { UV2: Zeipunkt, prä } \\
\text { vs. post vs. follow- } \\
\text { up (6 u. 12 Monate) } \\
\text { AV: Gewicht, FB } \\
\text { zum } \\
\text { Essverhaltensowie } \\
\text { Compliance }\end{array}$ & $\begin{array}{l}\text { Signifikante } \\
\text { Gewichtserhöhung in EG } \\
\text { im Vergleich zu KG1 und } \\
\text { KG2 } \\
\text { Signifikant höhere } \\
\text { Zufriedenheitund } \\
\text { Compliance in KG1 }\end{array}$ \\
\hline $\begin{array}{l}\text { Tate, Wing \& } \\
\text { Winett (2001) }\end{array}$ & $\begin{array}{l}\text { 6-monatiges } \\
\text { verhaltenstherapeut. } \\
\text { Progamm, E-Mail und } \\
\text { WWW-Angebot } \\
\text { (Tagebücher, } \\
\text { Verhaltenstraining) }\end{array}$ & $\begin{array}{l}\text { UV1: Betroffene } \\
\text { mit Adipositas: } \\
\text { Internet-VT-EG } \\
(\mathrm{n}=32) \text { vs. Internet } \\
\text { Informations-KG } \\
\text { (n=33) } \\
\text { UV2: Zeitpunkt, } \\
\text { prä vs. post vs. } \\
\text { follow-up (3 u. } 6 \\
\text { Monate) } \\
\text { AV: Gewicht }\end{array}$ & $\begin{array}{l}\text { Signifikante } \\
\text { Gewichtsreduktion in EG } \\
\text { im Vergleich zu KG }\end{array}$ \\
\hline
\end{tabular}


(Ott, 2003, S. 137: Studien zu Posttraumatischen Belastungsstörungen)

\begin{tabular}{|l|l|l|l|}
\hline Studie & Intervention & Stichprobe, Design & Ergebnisse \\
\hline Lange, van & WWW-Angebot, & UV1: subklinische & Signifikante Reduktion \\
den ven, & Selbstkonfrontation u. & Stichprobe: Internet- & der PTSD-Symptome \\
Schrieken \& & kognitive & EG (n=13) vs. Warte- & in EG im Vergleich zu \\
Emmelkamp & Umstrukturierung über & KG (n=12) & KG \\
$(2001)$ & schriftliche Essays, 5 & UV2: Zeitpunkt, prä vs. & \\
& Wochen & post vs. follow-up & \\
\hline $\begin{array}{l}\text { Lange, van } \\
\text { den Ven, }\end{array}$ & WWW-Angebot, & AV: Fragebögen & \\
Schrieken, & kognitive subklinische & Signifikante Reduktion \\
Bredeweg \& & Umstrukturierung über & UV2: Zeitpunkt, prä vs. & Verbesserung des \\
Emmelkamp & schriftliche Essays, 5 & post vs. 6-Wochen & allgemeinen \\
$($ 2001) & Wochen & follow up & Wohlbefindens \\
& & AV: Fragebogen & \\
\hline
\end{tabular}

(Ott, 2003, S. 138: Studien zu substanzbezogenen Störungen)

\begin{tabular}{|c|c|c|c|}
\hline Studie & Intervention & Stichprobe, Design & Ergebnisse \\
\hline $\begin{array}{l}\text { Feil, Noell, } \\
\text { Lichtenstein, } \\
\text { Boles \& } \\
\text { McKay } \\
(2003=\end{array}$ & $\begin{array}{l}\text { WWW-Angebot aus } \\
\text { kognitiv-behavioralen } \\
\text { Komponenten und } \\
\text { Diskussionsforen, } 3 \\
\text { Monate }\end{array}$ & $\begin{array}{l}\text { UV1: Betroffene mit } \\
\text { Nikotinabhängigkeit } \\
\text { (n=370), keine KG } \\
\text { UV2: Zeitpunkt, prä vs. } \\
\text { post } \\
\text { AV: Fragebögen zur } \\
\text { Zufriedenheit, } \\
\text { Abstinenzquote }\end{array}$ & $\begin{array}{l}\text { Signifikante Reduktion } \\
\text { der Anzahl der Raucher } \\
\text { Zufriedenheit mit } \\
\text { sozialer Unterstützung }\end{array}$ \\
\hline $\begin{array}{l}\text { Lenert, } \\
\text { Munoz, } \\
\text { Stoddard, } \\
\text { Delucchi, } \\
\text { Bansod, } \\
\text { Skoczen \& }\end{array}$ & $\begin{array}{l}\text { WWW-Angebot aus } \\
\text { kognitv-behavioralen } \\
\text { Komponenten u. } \\
\text { autom. E-Mail zur } \\
\text { Verhaltenskontrolle, } 8 \\
\text { Wochen }\end{array}$ & $\begin{array}{l}\text { UV1: Betroffene mit } \\
\text { Nikotinabhängigkeit } \\
(\mathrm{n}=49) \\
\text { UV2: Zeitpunkt, prä vs. } \\
\text { post } \\
\text { AV: Fragebögen zur }\end{array}$ & $\begin{array}{l}\text { Signifikante Reduktion } \\
\text { der Anzahl der Raucher } \\
\text { Hohe Dropout-Rate }\end{array}$ \\
\hline
\end{tabular}




\begin{tabular}{|c|c|c|c|}
\hline $\begin{array}{l}\text { Perez-Stable } \\
(2003)\end{array}$ & & $\begin{array}{l}\text { Zufriedenheit und zum } \\
\text { Rauchverhalten }\end{array}$ & \\
\hline $\begin{array}{l}\text { Woodruff, } \\
\text { Edwards, } \\
\text { Conway } \\
\& \text { Elliott } \\
(2001)\end{array}$ & $\begin{array}{l}\text { Moderierter Chat, } 7 \text { x } \\
\text { 1h, ausgebildete } \\
\text { Berater, klienten- } \\
\text { zentrierter Ansatz, } 2 \\
\text { Wochen }\end{array}$ & $\begin{array}{l}\text { UV1: jugendliche } \\
\text { Betroffene mit } \\
\text { Nikotinabhängigkeit } \\
\text { (n=11), keine KG } \\
\text { UV2: Zeitpunkt, prä vs. } \\
\text { post vs. 1-Monats } \\
\text { follow-up } \\
\text { AV: Fragebögen und - } \\
\text { einstellung }\end{array}$ & $\begin{array}{l}\text { Signifikante Reduktion } \\
\text { der Anzahl der Raucher } \\
\text { und Menge der } \\
\text { gerauchten Zigaretten }\end{array}$ \\
\hline
\end{tabular}

(Ott, 2003, S. 139: Studien zu verhaltensmedizinischen Störungen)

\begin{tabular}{|c|c|c|c|}
\hline Studie & Intervention & Stichprobe, Design & Ergebnisse \\
\hline $\begin{array}{l}\text { Andersson, } \\
\text { Stromgren, } \\
\text { Strom \& } \\
\text { Lyttkens } \\
(2002)\end{array}$ & $\begin{array}{l}\text { WWW-Angebot aus } \\
\text { kognitiv-behavioralen } \\
\text { Komponenten u. E- } \\
\text { Mail Versand, } 8 \\
\text { Wochen }\end{array}$ & $\begin{array}{l}\text { UV1: Patn. Mit } \\
\text { Tinnitus (>6 Monate), } \\
\text { Internet-EG (n=53), } \\
\text { Warte-KG (n=74) } \\
\text { UV2: Zeitpunkt, prä vs. } \\
\text { post, 1-Jahr follow-up } \\
\text { AV: Fragebögen zur } \\
\text { Tinnitus Belastung, } \\
\text { Zufriedenheit, } \\
\text { Tagebuch während des } \\
\text { Treatments }\end{array}$ & $\begin{array}{l}\text { Signifikante Reduktion } \\
\text { der Tinnitus bedingten } \\
\text { Belastung und } \\
\text { depressiven } \\
\text { Symptomatiken über } \\
\text { die Zeit und im Vgl. } \\
\text { zur KG } \\
\text { Hohe Dropout Rate } \\
(>50 \%) \text { in EG }\end{array}$ \\
\hline $\begin{array}{l}\text { Holden, } \\
\text { Bearison, } \\
\text { Rode, Kapi- } \\
\text { loff, } \\
\text { Rosenberg } \\
\& \\
\text { Rosenzweig } \\
(2002)\end{array}$ & $\begin{array}{l}\text { Interaktives Programm } \\
\text { mit kognitiv- } \\
\text { behavioralen Modulen }\end{array}$ & $\begin{array}{l}\text { UV1: jugendliche Patn. } \\
\text { Mit chronischen } \\
\text { Schmerzen: Internet- } \\
\text { EG (n=39), keine KG } \\
\text { UV2: zeitreihen- } \\
\text { analytische } \\
\text { Auswertung } \\
\text { AV: Selbstreportmaße } \\
\text { zu } \\
\text { Schmerzdimensionen u. } \\
\text { Angstsymptomen }\end{array}$ & $\begin{array}{l}\text { Nicht signifikante } \\
\text { Reduktion der } \\
\text { Schmerz- und } \\
\text { Angstsymptomatik in } \\
\text { der EG }\end{array}$ \\
\hline
\end{tabular}




\begin{tabular}{|l|l|l|l|}
\hline Krishna, & WWW-Angebot mit & UV1: jugendliche Patn. & Signifikante Erhöhung \\
Fransisco, & behavioralen & mit Asthma: Internet \& & des Asthma-Wissens in \\
Balas, Konig & Selbstmanagement- & Edukation EG (n=114) & der EG \\
\& Madsen & Komponenten & $\begin{array}{l}\text { vs. Edukation KG } \\
\text { (n=114) }\end{array}$ & $\begin{array}{l}\text { Signifikante Reduktion } \\
\text { der Asthma-Symptom- } \\
\text { UV2: Zeitpunkt prä vs. }\end{array}$ \\
Tag in der EG \\
\end{tabular}

(Ott, 2003, S. 140: Studien zu psychischen Problemen bei körperlichen Erkrankungen)

\begin{tabular}{|l|l|l|l|}
\hline Studie & Intervention & Stichprobe, Design & Ergebnisse \\
\hline Barrera, & WWW-Angebot, & UV1: Patn. Mit & Signifikante Erhöhung der \\
Glasgow, & Mailingliste u. & Diabetes II: Internet- & subjektiv wahrgenommen \\
McKay, & Diskussionsforen, E- & EG1 (n=40), Internet \& & sozialen Unterstützung in \\
Boles \& Feil & Mail, Ziel: soziale & f2fSelbstmanagement & EG1 und EG2 im Vgl. zu \\
$(2002)$ & Unterstützung; 3 & EG2 (n=40), f2f & den KG \\
& Monate & Selbstmanagement & \\
& & Training KG1 (n=40), & \\
& & Information KG2 & \\
& & (n=40) & \\
& & &
\end{tabular}




\begin{tabular}{|c|c|c|c|}
\hline & & $\begin{array}{l}\text { UV2: Zeitpunkt, prä vs. } \\
\text { post } \\
\text { AV: Fragebögen }\end{array}$ & \\
\hline $\begin{array}{l}\text { Fogel, } \\
\text { Albert, } \\
\text { Schnabel, } \\
\text { Ditkoff \& } \\
\text { Neugut } \\
(2003)\end{array}$ & $\begin{array}{l}\text { Mailinglisten u. } \\
\text { Diskussionsforen zum } \\
\text { Thema Brustkrebs }\end{array}$ & $\begin{array}{l}\text { UV1: Patn. Mit } \\
\text { Brustkrebs (n=188), ex- } \\
\text { post-facto } \\
\text { UV2: keine } \\
\text { AV: Fragebögen zur } \\
\text { sozialen Unterstützung } \\
\text { und Einsamkeit }\end{array}$ & $\begin{array}{l}42 \% \text { aller Befragten nutzen } \\
\text { Internetangebote } \\
\text { Signifikante Erhöhung der } \\
\text { subjektiv } \\
\text { wahrgenommenen sozialen } \\
\text { Unterstützung bei } \\
\text { Internetnutzerinnen } \\
\text { Signifikant niedrigere } \\
\text { Einsamkeitseinschätzungen } \\
\text { bei Internetnutzerinnen }\end{array}$ \\
\hline $\begin{array}{l}\text { McKay, } \\
\text { Glasgow, } \\
\text { Feil, Boles } \\
\text { \& Barrera } \\
(2002)\end{array}$ & $\begin{array}{l}\text { WWW-Angebot mit } \\
\text { edukativen u. } \\
\text { Selbstmanagement } \\
\text { Modulen, } 3 \text { Monate }\end{array}$ & $\begin{array}{l}\text { UV1: Patn. Mit } \\
\text { Diabetes II, Internet- } \\
\text { EG (n=50), f2f-KG } \\
(\mathrm{n}=52) \\
\text { UV2:Zeitpunkt, prä vs. } \\
\text { post } \\
\text { AV: Fragbögen zum } \\
\text { Diätverhalten u. } \\
\text { Allgemeinen } \\
\text { Wohlbefinden, } \\
\text { physiologische Maße }\end{array}$ & $\begin{array}{l}\text { Signifikante Verbesserung } \\
\text { des Diätverhaltens, des } \\
\text { Wohlbefindens u. in den } \\
\text { physiologischen Variablen } \\
\text { Keine Unterschiede in den } \\
\text { Gruppe }\end{array}$ \\
\hline $\begin{array}{l}\text { Tate, } \\
\text { Jackvony \& } \\
\text { WIng (2003) }\end{array}$ & $\begin{array}{l}\text { WWW-Angebot mit } \\
\text { edukativen u. } \\
\text { behavioralen Modulen } \\
\text { sowie individuelle E- } \\
\text { Mail-Beratung, Ziel: } \\
\text { Gewichtsreduktion, } 1 \\
\text { Jahr }\end{array}$ & $\begin{array}{l}\text { UV1: Pers. Mit Risiko } \\
\text { Diabetes II, WWW- } \\
\text { Angebot EG1 (n=46), } \\
\text { WWW-Angebot u. E- } \\
\text { Mail EG2 (n=46), } \\
\text { keine KG }\end{array}$ & $\begin{array}{l}\text { Signifikant höhere } \\
\text { Gewichtsreduktion in EG2 } \\
\text { mit individueller Beratung }\end{array}$ \\
\hline $\begin{array}{l}\text { Winzelberg, } \\
\text { Classen, } \\
\text { Alpers, } \\
\text { Roberts, } \\
\text { Koopman, }\end{array}$ & $\begin{array}{l}\text { Newsgruppen-basierte } \\
\text { Selbsthilfegruppe, } \\
\text { moderiert, } 12 \text { Wochen }\end{array}$ & $\begin{array}{l}\text { UV1: Patn. mit } \\
\text { Brustkrebs }(n=72) \text {, } \\
\text { keine KG } \\
\text { UV2: Zeitpunkt, prä vs. } \\
\text { post }\end{array}$ & $\begin{array}{l}\text { Signifikante Reduktion der } \\
\text { depressiven Symptome, } \\
\text { des wahrgenommenen } \\
\text { Stress u. der Krebs } \\
\text { bedingten Trauma- }\end{array}$ \\
\hline
\end{tabular}




\begin{tabular}{|l|l|l|}
\hline Adams, & AV: Fragebögen zur & Symptome \\
Ernst, Dev & sozialen Unterstützung, & \\
\& Taylor & psychisches & \\
$(2003)$ & Wohlbefinden & \\
\hline
\end{tabular}


Christoph Barthel

\section{Persönliche Daten}

Geburtsdatum:

18.06. 1974

Geburtsort:

Familienstand:

Hamm

ledig

\section{Schulausbildung}

August 1984 - Juni 1994

Gymnasium Hammonense, Hamm

\section{Studium}

Oktober 1994 - März 2001

Studium der Humanmedizin an der Rheinisch-

Westfälisch-Technischen Hochschule Aachen

\section{Berufliche Laufbahn}

Juli 2001 - Dezember 2002

Januar 2003 - Dezember 2005

Januar 2006 - April 2006

Mai 2006 - Juni 2007

Juni 2007
Anstellung als Arzt im Praktikum im Westfälischen Zentrum für Psychiatrie und Psychotherapie, Universitätsklinik Bochum

Weiterbeschäftigung als Assistenzarzt in o.g. Hause

Assistenzärztliche Betreuung der Spezialambulanz für „Psychische Störungen nach Arbeitsunfällen“ in der neurologischen Klinik Bergmannsheil, Bochum

Tätigkeit als Assistenzarzt in nervenärztlicher Praxis Drs. Gebauer und von Rhein, Bottrop

Facharztprüfung im Fach Psychiatrie und Psychotherapie

Niederlassung als ärztlicher Psychotherapeut in Bottrop 